\title{
REGULARITY AND CONTINUITY OF THE MULTILINEAR STRONG MAXIMAL OPERATORS
}

\author{
FENG LIU, QINGYING XUE*, AND KÔZÔ YABUTA
}

\begin{abstract}
Let $m \geq 1$, in this paper, our object of investigation is the regularity and and continuity properties of the following multilinear strong maximal operator

$$
\mathscr{M}_{\mathcal{R}}(\vec{f})(x)=\sup _{\substack{R \ni x \\ R \in \mathcal{R}}} \prod_{i=1}^{m} \frac{1}{|R|} \int_{R}\left|f_{i}(y)\right| d y,
$$

where $x \in \mathbb{R}^{d}$ and $\mathcal{R}$ denotes the family of all rectangles in $\mathbb{R}^{d}$ with sides parallel to the axes. When $m=1$, denote $\mathscr{M}_{\mathcal{R}}$ by $\mathcal{M}_{\mathcal{R}}$. Then, $\mathcal{M}_{\mathcal{R}}$ coincides with the classical strong maximal function initially studied by Jessen, Marcinkiewicz and Zygmund. We showed that $\mathscr{M}_{\mathcal{R}}$ is bounded and continuous from the Sobolev spaces $W^{1, p_{1}}\left(\mathbb{R}^{d}\right) \times \cdots \times W^{1, p_{m}}\left(\mathbb{R}^{d}\right)$ to $W^{1, p}\left(\mathbb{R}^{d}\right)$, from the Besov spaces $B_{s}^{p_{1}, q}\left(\mathbb{R}^{d}\right) \times \cdots \times B_{s}^{p_{m}, q}\left(\mathbb{R}^{d}\right)$ to $B_{s}^{p, q}\left(\mathbb{R}^{d}\right)$, from the Triebel-Lizorkin spaces $F_{s}^{p_{1}, q}\left(\mathbb{R}^{d}\right) \times \cdots \times F_{s}^{p_{m}, q}\left(\mathbb{R}^{d}\right)$ to $F_{s}^{p, q}\left(\mathbb{R}^{d}\right)$. As a consequence, we further showed that $\mathscr{M}_{\mathcal{R}}$ is bounded and continuous from the fractional Sobolev spaces $W^{s, p_{1}}\left(\mathbb{R}^{d}\right) \times \cdots \times W^{s, p_{m}}\left(\mathbb{R}^{d}\right)$ to $W^{s, p}\left(\mathbb{R}^{d}\right)$ for $0<s \leq 1$ and $1<p<\infty$. As an application, we obtain a weak type inequality for the Sobolev capacity, which can be used to prove the p-quasicontinuity of $\mathscr{M}_{\mathcal{R}}$. In addition, we proved that $\mathscr{M}_{\mathcal{R}}(\vec{f})$ is approximately differentiable a.e. if $\vec{f}=\left(f_{1}, \ldots, f_{m}\right)$ with each $f_{j} \in L^{1}\left(\mathbb{R}^{d}\right)$ being approximately differentiable a.e. The discrete type of the strong maximal operators has also been considered. We showed that this discrete type of the maximal operators enjoys somewhat unexpected regularity properties.
\end{abstract}

\section{IntroduCtion}

1.1. Hardy-Littlewood maximal functions. Let $f \in L_{\text {loc }}^{1}\left(\mathbb{R}^{d}\right)$ with $d \geq 1$ and $\mathcal{M}$ be the well-known Hardy-Littlewood maximal operator defined on $\mathbb{R}^{n}$ as follows.

$$
\mathcal{M} f(x)=\sup _{r>0} \frac{1}{\left|B_{r}(x)\right|} \int_{B_{r}(x)}|f(y)| d y,
$$

where $B_{r}(x)$ is the open ball in $\mathbb{R}^{d}$ centered at $x$ with radius $r$ and $\left|B_{r}(x)\right|$ denotes the volume of $B_{r}(x)$. Analogously, the uncentered maximal function $\widetilde{\mathcal{M}} f$ at a point $x$ is defined by taking the supremum of averages over open balls that contain the point. It was well known that the maximal functions and their purpose in differentiation on $\mathbb{R}$ were first introduced by Hardy and Littlewood [24], and on $\mathbb{R}^{d}$ were treated by Wiener [55]. The celebrated theorem of HardyLittlewood-Wiener states that the operator $\mathcal{M}$ is of type $(p, p)$ for $1<p \leq \infty$ and weak type $(1,1)$. As a basic and important tool in Harmonic analysis and other fields, such as PDE, the

Key words and phrases. Multilinear strong maximal operators, discrete multilinear strong maximal operators, Sobolev spaces, regularity, Tribel-Lizorkin spaces and Besov spaces, mixed Lebesgue spaces and Sobolev spaces, Sobolev capacity.

The first author was supported partly by NSFC (No. 11701333) and SP-OYSTTT-CMSS (No. Sxy2016K01). The second author was partly supported by NSFC (Nos. 11471041, 11671039) and NSFC-DFG (No. 11761131002). The third named author was supported partly by Grant-in-Aid for Scientific Research (C) Nr. 15K04942, Japan Society for the Promotion of Science.

Corresponding author: Qingying Xue Email: qyxue@bnu.edu.cn. 
maximal functions and their variants are often used to control some other important operators and give some good absolute size estimates (see [7], [35] and [36]).

There is a basic question in the theory of Hardy-Littlewood maximal operators: How does the Hardy-Littlewood maximal operator preserve the smoothness properties of a function? Achievements have been made in this direction in the past few years. Among them is the nice work of Kinnunen [29] in 1997, where the regularity properties of maximal operators on the $W^{1, p}$ spaces has been studied. Recall that the Sobolev spaces $W^{1, p}\left(\mathbb{R}^{d}\right), 1 \leq p \leq \infty$, are defined by

$$
W^{1, p}\left(\mathbb{R}^{d}\right):=\left\{f: \mathbb{R}^{d} \rightarrow \mathbb{R}:\|f\|_{1, p}=\|f\|_{L^{p}\left(\mathbb{R}^{d}\right)}+\|\nabla f\|_{L^{p}\left(\mathbb{R}^{d}\right)}<\infty\right\},
$$

where $\nabla f=\left(D_{1} f, \ldots, D_{d} f\right)$ is the weak gradient of $f$. Kinnunen showed that $\mathcal{M}$ is bounded from $W^{1, p}\left(\mathbb{R}^{d}\right)$ to $W^{1, p}\left(\mathbb{R}^{d}\right)$ for $1<p \leq \infty$. It was noticed that the $W^{1, p}$-bound for $\widetilde{\mathcal{M}}$ also holds by a simple modification of Kinnunen's arguments or Theorem 1 of 23 . Later on, the result of Kinnunen has been extended to a local version in [30, to a fractional version in [31, to a multisublinear version in [12, 41] and to a one-sided version in [40. Whether the continuity for $\mathcal{M}$ on $W^{1, p}\left(\mathbb{R}^{d}\right)$ space holds or not is another certainly nontrivial problem, since the maximal operator is not necessarily sublinear at the derivative level. This problem was first posed by Hajłasz and Onninen [23] and was later settled affirmatively by Luiro [46].

Due to the lack of reflexivity of $L^{1}$, it makes the understanding of the $W^{1,1}\left(\mathbb{R}^{d}\right)$ regularity more subtler. One interesting question was raised by Hajłasz and Onninen in [23]: Is the operator $f \mapsto|\nabla \mathcal{M} f|$ bounded from $W^{1,1}\left(\mathbb{R}^{d}\right)$ to $L^{1}\left(\mathbb{R}^{d}\right)$ ? A complete answer was addressed only in dimension $d=1$ in [2, 34, 39, 51] and partial progress on the general case $d \geq 2$ was given by Hajłasz and Malý [22] and Luiro [48. For more previous works or related topic we refer the readers to consult [3, 10, 11, 13, 34, 38, 42, and the references therein.

Now we know that $\mathcal{M}$ is bounded on $L^{p}\left(\mathbb{R}^{d}\right)=W^{0, p}\left(\mathbb{R}^{d}\right)$ and $W^{1, p}\left(\mathbb{R}^{d}\right)$ for $p>1$. Therefore a natural question arises: what is the properties of $\mathcal{M}$ on the fractional Sobolev spaces $W^{s, p}\left(\mathbb{R}^{d}\right)$ defined by the Bessel potentials when $0<s<1$ ? This question was first studied by Korry 33 . who observed that $\mathcal{M}: W^{s, p}\left(\mathbb{R}^{d}\right) \rightarrow W^{s, p}\left(\mathbb{R}^{d}\right)$ is bounded for all $0<s<1$ and $1<p<\infty$. Notice that $F_{s}^{p, 2}\left(\mathbb{R}^{d}\right)=W^{s, p}\left(\mathbb{R}^{d}\right)$ for any $s>0$ and $1<p<\infty$ (see [20]). It may be further expected that $\mathcal{M}$ still enjoys the boundedness on Triebel-Lizorkin spaces $F_{s}^{p, q}\left(\mathbb{R}^{d}\right)$. This was done by Korry [32, who indeed proved that $\mathcal{M}$ is bounded on the inhomogeneous TriebelLizorkin spaces $F_{s}^{p, q}\left(\mathbb{R}^{d}\right)$ and Besov spaces $B_{s}^{p, q}\left(\mathbb{R}^{d}\right)$ for all $0<s<1$ and $1<p, q<\infty$. Recently, Luiro [47] established the continuity of $\mathcal{M}$ on $F_{s}^{p, q}\left(\mathbb{R}^{d}\right)$ for all $0<s<1$ and $1<$ $p, q<\infty$. Still more recently, Liu and Wu [44 extended the above results to the maximal operators associated with polynomial mappings.

1.2. Multilinear strong maximal operators. Over the past few decades, many celebrated works have been done in the study of the maximal functions associated with different kinds of basis. These bases mainly including: some differentiation bases (balls or cubes, rectangles with some restrictions see [25], [57] and [58]), translation in-variant basis of rectangles [14], basis formed by convex sets, using rectangles with a side parallel to some direction (lacunary parabolic set of directions in [50, Cantor set of directions in [26, arbitrary set of directions [1], [27]). In this paper, we will focus on the translation in-variant basis of rectangles studied by Córdoba and Fefferman [14].

Let $\vec{f}=\left(f_{1}, \ldots, f_{m}\right)$ be an $m$-dimensional vector of locally integrable functions and $\mathcal{R}$ denotes the collection of all open rectangles $R \subset \mathbb{R}^{d}$ with sides parallel to the coordinate axes. In 2011, Grafakos, Liu, Pérez and Torres [21] introduced and studied the weighted strong and endpoint 
estimates for the multilinear strong maximal function $\mathscr{M}_{\mathcal{R}}$, which is defined by

$$
\mathscr{M}_{\mathcal{R}}(\vec{f})(x)=\sup _{\substack{R \ni x \\ R \in \mathcal{R}}} \prod_{i=1}^{m} \frac{1}{|R|} \int_{R}\left|f_{i}\left(y_{i}\right)\right| d y_{i},
$$

where $x \in \mathbb{R}^{d}$ and $\mathcal{R}$ denotes the family of all rectangles in $\mathbb{R}^{d}$ with sides parallel to the axes.

Whenever $m=1$, we simply denote $\mathscr{M}_{\mathcal{R}}$ by $\mathcal{M}_{\mathcal{R}}$. Then $\mathcal{M}_{\mathcal{R}}$ coincides with the classical strong maximal operator. As the most prototypical representative of the multi-parameter operators, $\mathcal{M}_{\mathcal{R}}$ can be looked as a geometric maximal operator which commutes with full $d$-parameter group of dilations $\left(x_{1}, x_{2}, \ldots, x_{d}\right) \rightarrow\left(\delta_{1} x_{1}, \delta_{2} x_{2}, \ldots, \delta_{d} x_{d}\right)$. It was proved by García-Cuerva and Rubio de Francia that $\mathcal{M}_{\mathcal{R}}$ is bounded on $L^{p}\left(\mathbb{R}^{d}\right)$ for all $1<p<\infty$ (see [17, p.452]). In 1935, a maximal theorem was given by Jessen, Marcinkiewicz and Zygmund in 25]. They pointed out that unlike the classical Hardy-Littlewood maximal operator, the strong maximal function is not of weak type $(1,1)$. As a replacement, they showed that it is bounded from $L\left(\log ^{+} L\right)\left(\mathbb{R}^{d}\right)$ to $L^{1}\left(\mathbb{R}^{d}\right)$. Subsequently, an additional proof of the maximal theorem was given by Córdoba and Fefferman in 1975, using an alternative geometric method [14]. The basis of the work of Córdoba and Fefferman is a selection theorem for families of rectangles in $\mathbb{R}^{d}$. Some delicate properties of rectangles in $\mathbb{R}^{d}$ were also quantified in that study.

Furthermore, if $m=1$ and $d=1$, the operator $\mathscr{M}_{\mathcal{R}}=\widetilde{\mathcal{M}}$. It was known that $\widetilde{\mathcal{M}}$ is bounded and continuous on $W^{1, p}(\mathbb{R})$ for $1<p<\infty$. It follows from [2, 39] that if $f \in W^{1,1}(\mathbb{R})$, then $\widetilde{\mathcal{M}} f$ is absolutely continuous on $\mathbb{R}$ and it holds that $\left\|(\widetilde{\mathcal{M}} f)^{\prime}\right\|_{L^{1}(\mathbb{R})} \leq\left\|f^{\prime}\right\|_{L^{1}(\mathbb{R})}$. For $d \geq 1$, Aldaz and Pérez Lázaro [3] considered a class of local strong maximal operator and proved that it maps $\mathrm{BV}(U)$ into $L^{1}(U)$, where $U$ is an open set of $\mathbb{R}^{d}$ and $\mathrm{BV}(U)$ is a subclass of $L^{1}(U)$ functions. See [19, Definition 1.3] and [4, Definition 3.4] for instance.

The results in [21] indicate that $\mathscr{M}_{\mathcal{R}}$ is bounded from $L^{p_{1}}\left(\mathbb{R}^{d}\right) \times \cdots \times L^{p_{m}}\left(\mathbb{R}^{d}\right)$ to $L^{p}\left(\mathbb{R}^{d}\right)$ for all $1<p_{1}, \ldots, p_{m}, p \leq \infty$ and $1 / p=\sum_{i=1}^{m} 1 / p_{i}$. Moreover, for $\vec{f}=\left(f_{1}, \ldots, f_{m}\right)$ with each $f_{i} \in L^{p_{i}}\left(\mathbb{R}^{d}\right)$, the following norm inequality holds

$$
\left\|\mathscr{M}_{\mathcal{R}}(\vec{f})\right\|_{L^{p}\left(\mathbb{R}^{d}\right)} \lesssim p_{1, \ldots, p_{m}} \prod_{i=1}^{m}\left\|f_{i}\right\|_{L^{p_{i}\left(\mathbb{R}^{d}\right)}} .
$$

It is well known that the geometry of rectangles in $\mathbb{R}^{d}$ is more intricate than that of cubes or balls, even when both classes of sets are restricted to have sides parallel to the axes. Even for $m=1$, a basic observation is that $\mathcal{M} f(x) \lesssim_{d} \mathcal{M}_{\mathcal{R}} f(x)$ for all $x \in \mathbb{R}^{d}$. However, there does not exist any constant $C>0$ such that $\mathcal{M}_{\mathcal{R}} f(x) \leq C \mathcal{M} f(x)$ for all $x \in \mathbb{R}^{d}$. This indicates fully that the strong maximal functions are uncontrollable. For these reasons, this makes the investigation of the strong maximal functions very complex, but also quite interesting.

Based on the facts concerning the previous results on the Hardy-Littlewood maximal operators, it is therefore a natural question to ask whether the multilinear strong maximal operators are bounded and continuous on the products of the first order Sobolev spaces $W^{1, p}\left(\mathbb{R}^{d}\right)$ or the fractional Sobolev spaces $W^{s, p}\left(\mathbb{R}^{d}\right)$ or on its generalizations $F_{s}^{p, q}\left(\mathbb{R}^{d}\right)$ and $B_{s}^{p, q}\left(\mathbb{R}^{d}\right)$. This is the main motivation of this work. In the first part of this work, the regularityand continuity properties of the strong maximal functions will be studied. We will show that $\mathscr{M}_{\mathcal{R}}$ is bounded and continuous from the Sobolev spaces $W^{1, p_{1}}\left(\mathbb{R}^{d}\right) \times \cdots \times W^{1, p_{m}}\left(\mathbb{R}^{d}\right)$ to $W^{1, p}\left(\mathbb{R}^{d}\right)$, from the Besov spaces $B_{s}^{p_{1}, q}\left(\mathbb{R}^{d}\right) \times \cdots \times B_{s}^{p_{m}, q}\left(\mathbb{R}^{d}\right)$ to $B_{s}^{p, q}\left(\mathbb{R}^{d}\right)$, from the Triebel-Lizorkin spaces $F_{s}^{p_{1}, q}\left(\mathbb{R}^{d}\right) \times \cdots \times F_{s}^{p_{m}, q}\left(\mathbb{R}^{d}\right)$ to $F_{s}^{p, q}\left(\mathbb{R}^{d}\right)$. We further showed that $\mathscr{M}_{\mathcal{R}}$ is bounded and continuous from the fractional Sobolev spaces $W^{s, p_{1}}\left(\mathbb{R}^{d}\right) \times \cdots \times W^{s, p_{m}}\left(\mathbb{R}^{d}\right)$ to $W^{s, p}\left(\mathbb{R}^{d}\right)$ for $0<s<1$ and $1<p<\infty$. As an application, we obtain a weak type inequality for the Sobolev capacity, which can be used to prove $p$-quasicontinuity of the strong maximal function of a Sobolev function. In 
addition, we also show that $\mathscr{M}_{\mathcal{R}}(\vec{f})$ is approximately differentiable a.e. if $\vec{f}=\left(f_{1}, \ldots, f_{m}\right)$ with each $f_{j} \in L^{1}\left(\mathbb{R}^{d}\right)$ being approximately differentiable a.e.

1.3. Discrete multilinear strong maximal operators. Another aim of this paper is to investigate the regularity properties of the discrete multilinear strong maximal operators. For a vector-valued function $\vec{f}=\left(f_{1}, \ldots, f_{m}\right)$ with each $f_{j}$ being a discrete function defined on $\mathbb{Z}^{d}$, we define the discrete multilinear strong maximal operator $\mathbb{M}_{\mathcal{R}}$ by

$$
\mathbb{M}_{\mathcal{R}}(\vec{f})(\vec{n})=\sup _{\substack{R \ni \vec{n} \\ R \in \mathcal{R}}} \frac{1}{N(R)^{m}} \prod_{i=1}^{m} \sum_{\vec{k} \in R \cap \mathbb{Z}^{n}}\left|f_{i}(\vec{k})\right|,
$$

where $N(R)$ is the number of elements in the set $R \cap \mathbb{Z}^{d}$. When $m=1$, the operator $\mathbb{M}_{\mathcal{R}}$ reduces to the discrete strong maximal operator $M_{\mathcal{R}}$.

Let us recall some pertinent definitions, notations and backgrounds. We shall generally denote by $\vec{n}=\left(n_{1}, n_{2}, \ldots, n_{d}\right)$ a vector in $\mathbb{Z}^{d}$. For a discrete function $f: \mathbb{Z}^{d} \rightarrow \mathbb{R}$, we define the $\ell^{p}\left(\mathbb{Z}^{d}\right)$ norm for $1 \leq p<\infty$ by $\|f\|_{\ell^{p}\left(\mathbb{Z}^{d}\right)}=\left(\sum_{\vec{n} \in \mathbb{Z}^{d}}|f(\vec{n})|^{p}\right)^{1 / p}$ and $\ell^{\infty}\left(\mathbb{Z}^{d}\right)$-norm by $\|f\|_{\ell^{\infty}\left(\mathbb{Z}^{d}\right)}=$ $\sup _{\vec{n} \in \mathbb{Z}^{d}}|f(\vec{n})|$. Next, we recall the definitions of discrete Sobolev space $W^{1, p}\left(\mathbb{Z}^{d}\right)$ and $\mathrm{BV}_{q}\left(\mathbb{Z}^{d}\right)$ function class.

Definition 1.1 (Discrete Sobolev space $W^{1, p}\left(\mathbb{Z}^{d}\right)$, (6])). For $1 \leq l \leq d$, let $\vec{e}_{l}$ be the canonical $l$-th base vector defined by $\vec{e}_{l}=(0, \ldots, 0,1,0, \ldots, 0)$. Let $D_{l} f(\vec{n})$ be the partial derivative of $f$ given by $D_{l} f(\vec{n})=f\left(\vec{n}+\vec{e}_{l}\right)-f(\vec{n})$ and $\nabla f$ be the gradient of $f$ defined by $\nabla f(\vec{n})=\left(D_{1} f(\vec{n}), \ldots, D_{d} f(\vec{n})\right)$. Then, the discrete Sobolev spaces is defined by

$$
W^{1, p}\left(\mathbb{Z}^{d}\right):=\left\{f: \mathbb{Z}^{d} \rightarrow \mathbb{R} \mid\|f\|_{1, p}=\|f\|_{\ell^{p}\left(\mathbb{Z}^{d}\right)}+\|\nabla f\|_{\ell^{p}\left(\mathbb{Z}^{d}\right)}<\infty\right\} .
$$

Note that

$$
\|\nabla f\|_{\ell^{p}\left(\mathbb{Z}^{d}\right)} \leq 2 d\|f\|_{\ell^{p}\left(\mathbb{Z}^{d}\right)} \text { for } 1 \leq p \leq \infty
$$

It follows that

$$
\|f\|_{\ell^{p}\left(\mathbb{Z}^{d}\right)} \leq\|f\|_{1, p} \leq(2 d+1)\|f\|_{\ell^{p}\left(\mathbb{Z}^{d}\right)} \text { for } 1 \leq p \leq \infty .
$$

This implies that the discrete Sobolev space $W^{1, p}\left(\mathbb{Z}^{d}\right)$ is just $\ell^{p}\left(\mathbb{Z}^{d}\right)$ with an equivalent norm. It might make our efforts to study the $W^{1, p}\left(\mathbb{Z}^{d}\right)$ regularity of discrete maximal operators seem almost vacuous since any $\ell^{p}$-bound automatically implies a $W^{1, p}$-bound. However, the endpoint $p=1$ is highly nontrivial because of the lack of $\ell^{1}$-bound for discrete strong maximal operators.

To investigate the endpoint regularity of $\mathbb{M}_{\mathcal{R}}$, we now introduce the following function class.

Definition $1.2\left(\mathrm{BV}\left(\mathbb{Z}^{d}\right)\right.$ function class, $\left.([9])\right)$. We denote by $\mathrm{BV}\left(\mathbb{Z}^{d}\right)$ the set of all functions of bounded variation defined on $\mathbb{Z}^{d}$, where the total variation of $f: \mathbb{Z}^{d} \rightarrow \mathbb{R}$ is defined by

$$
\operatorname{Var}(f)=\|\nabla f\|_{\ell^{1}\left(\mathbb{Z}^{d}\right)} .
$$

(1.4) together with (1.5) and a simple example $f(\vec{n})=1$ yields that

$$
\mathrm{BV}\left(\mathbb{Z}^{d}\right) \subsetneq \ell^{1}\left(\mathbb{Z}^{d}\right)=W^{1,1}\left(\mathbb{Z}^{d}\right) .
$$

Recently, the investigation of the regularity of discrete maximal operators has also attracted the attention of many authors (see [6, 9, 10, 37, 40, 43, 45, 49, 52, et al.). Recall that the discrete uncentered version of maximal function is defined by

$$
M f(\vec{n})=\sup _{r>0, \vec{n} \in B_{r}} \frac{1}{N\left(B_{r}\right)} \sum_{\vec{k} \in B_{r} \cap \mathbb{Z}^{d}}|f(\vec{k})|,
$$


where the surpremum is taken over all open balls $B_{r}$ in $\mathbb{R}^{d}$ containing the point $\vec{n}$ with radius $r$ and $N\left(B_{r}\right)$ denotes the number of lattice points in the set $B_{r}$. We denote the centered version of discrete maximal function by $\widetilde{M}$.

When $d=1$, the regularity properties of the discrete maximal type operators were studied by Bober et al. [6], Temur [52] and Madrid [49, Carneiro and Madrid [10] and Liu [37. The following sharp inequalities have been established.

$$
\operatorname{Var}(\widetilde{M} f) \leq \operatorname{Var}(f)
$$

and

$$
\operatorname{Var}(M f) \leq 2\|f\|_{\ell^{1}(\mathbb{Z})} .
$$

For $d \geq 1$, Carneiro and Hughes [9] proved that $M$ maps $\ell^{1}\left(\mathbb{Z}^{d}\right)$ into $\mathrm{BV}\left(\mathbb{Z}^{d}\right)$ boundedly and continuously. In (1.3), if one replace the rectangles $R$ by balls $B_{r}$, then we denote $\mathbb{M}_{\mathcal{R}}$ by $\mathfrak{M}$. Still more recently, the results in [9] was extended by Liu and $\mathrm{Wu}[43$ ] as follows.

Theorem A $([43])$. Let $d \geq 1$. Then $\mathfrak{M}$ maps $\ell^{1}\left(\mathbb{Z}^{d}\right) \times \cdots \times \ell^{1}\left(\mathbb{Z}^{d}\right)$ into $\mathrm{BV}\left(\mathbb{Z}^{d}\right)$ boundedly and continuously.

It is observed that $\mathfrak{M}(\vec{f})(\vec{n}) \lesssim_{d, m} \mathbb{M}_{\mathcal{R}}(\vec{f})(\vec{n})$ for all $\vec{n} \in \mathbb{Z}^{d}$. Specially, $\mathbb{M}_{\mathcal{R}}=\mathfrak{M}$ when $d=1$. However, when $d \geq 2$, there does not exist any constant $C>0$ such that $\mathbb{M}_{\mathcal{R}}(\vec{f})(\vec{n}) \leq C \mathfrak{M}(\vec{f})(\vec{n})$ for all $\vec{n} \in \mathbb{Z}^{d}$. Based on the above analysis, it is interesting and natural to ask whether the discrete strong maximal operators still enjoy some sort of regularity properties. We will show that the discrete type of the strong maximal operators does enjoy somewhat unexpected regularities in the end of next part.

1.4. Main results. We now state our main results as follows.

Theorem 1.1 (Properties on Sobolev spaces). Let $1<p_{1}, \ldots, p_{m}, p<\infty$ and $1 / p=$ $\sum_{i=1}^{m} 1 / p_{i}$. Then $\mathscr{M}_{\mathcal{R}}$ is bounded and continuous from $W^{1, p_{1}}\left(\mathbb{R}^{d}\right) \times \cdots \times W^{1, p_{m}}\left(\mathbb{R}^{d}\right)$ to $W^{1, p}\left(\mathbb{R}^{d}\right)$. Moreover, if $\vec{f}=\left(f_{1}, \ldots, f_{m}\right)$ with each $f_{i} \in W^{1, p_{i}}\left(\mathbb{R}^{d}\right)$, then, for $1 \leq l \leq d$, it holds that

$$
\left|D_{l} \mathscr{M}_{\mathcal{R}}(\vec{f})(x)\right| \lesssim_{m, d, p_{1}, \ldots, p_{m}} \sum_{\mu=1}^{m} \mathscr{M}_{\mathcal{R}}\left(\vec{f}_{\mu}^{l}\right)(x) \text {, a.e. } x \in \mathbb{R}^{d},
$$

where $\vec{f}_{\mu}^{l}=\left(f_{1}, \ldots, f_{\mu-1}, D_{l} f_{\mu}, f_{\mu+1}, \ldots, f_{m}\right)$.

Remark 1.3. The case $p=\infty$ is also valid in Theorem 1.1, which follows from the similar arguments to those used in [29, Remark (iii)].

Theorem 1.2 (Properties on Besov spaces). Let $1<p_{1}, \ldots, p_{m}, p, q<\infty, 1 / p=\sum_{i=1}^{m} 1 / p_{i}$ and $0<s<1$. Then $\mathscr{M}_{\mathcal{R}}$ is bounded and continuous from $B_{s}^{p_{1}, q}\left(\mathbb{R}^{d}\right) \times \cdots \times B_{s}^{p_{m}, q}\left(\mathbb{R}^{d}\right)$ to $B_{s}^{p, q}\left(\mathbb{R}^{d}\right)$.

Theorem 1.3 (Properties on Triebel-Lizorkin spaces). Let $1<p_{1}, \ldots, p_{m}, p, q<\infty$, $1 / p=\sum_{i=1}^{m} 1 / p_{i}$ and $0<s<1$. Then $\mathscr{M}_{\mathcal{R}}$ is bounded and continuous from $F_{s}^{p_{1}, q}\left(\mathbb{R}^{d}\right) \times \cdots \times$ $F_{s}^{p_{m}, q}\left(\mathbb{R}^{d}\right)$ to $F_{s}^{p, q}\left(\mathbb{R}^{d}\right)$.

Noting that $F_{s}^{p, 2}\left(\mathbb{R}^{d}\right)=W^{s, p}\left(\mathbb{R}^{d}\right)$ for any $s>0$ and $1<p<\infty$, then Theorem 1.3 implies the following result immediately.

Corollary 1.4 (Properties on Fractional Sobolev spaces). Let $1<p_{1}, \ldots, p_{m}, p<\infty$, $1 / p=\sum_{i=1}^{m} 1 / p_{i}$ and $0<s<1$. Then $\mathscr{M}_{\mathcal{R}}$ is bounded and continuous from the fractional Sobolev spaces $W^{s, p_{1}}\left(\mathbb{R}^{d}\right) \times \cdots \times W^{s, p_{m}}\left(\mathbb{R}^{d}\right)$ to $W^{s, p}\left(\mathbb{R}^{d}\right)$. 
Theorem 1.1 can be used to obtain a weak type inequality for the Sobolev capacity, which can be further employed to prove the quasicontinuity of the strong maximal function of a Sobolev function. We first need to give the definition of Sobolev $p$-capacity.

Definition 1.4 (Sobolev $p$-capacity, ([28])). For $1<p<\infty$, the Sobolev $p$-capacity of the set $E \subset \mathbb{R}^{d}$ is defined by

$$
C_{p}(E):=\inf _{f \in \mathcal{A}(E)} \int_{\mathbb{R}^{d}}\left(|f(y)|^{p}+|\nabla f(y)|^{p}\right) d y,
$$

where $\mathcal{A}(E)=\left\{f \in W^{1, p}\left(\mathbb{R}^{d}\right): f \geq 1\right.$ on a neighbourhood of $\left.E\right\}$. We set $C_{p}(E)=\infty$ if $\mathcal{A}(E)=\emptyset$.

It was shown in [15] that the Sobolev $p$-capacity is a monotone and a countably subadditive set function. Also, it is an outer measure over $\mathbb{R}^{d}$.

Definition 1.5 (p-quasicontinuous and $p$-quasieverywhere, [15]). A function $f$ is said to be $p$-quasicontinuous in $\mathbb{R}^{d}$ if for every $\epsilon>0$, there exists a set $F \subset \mathbb{R}^{d}$ such that $C_{p}(F)<\epsilon$ and the restriction of $f$ to $\mathbb{R}^{d} \backslash F$ is continuous and finite. A property holds $p$-quasieverywhere if it holds outside a set of the Sobolev p-capacity zero.

Remark 1.6. It was known that each Sobolev function has a quasicontinuous representative, that is, for each $u \in W^{1, p}\left(\mathbb{R}^{d}\right)$, there is a $p$-quasicontinuous function $v \in W^{1, p}\left(\mathbb{R}^{d}\right)$ such that $u=v$ a.e. in $\mathbb{R}^{d}$. This representative is unique in the sense that if $v$ and $w$ are $p$-quasicontinuous and $v=w$ a.e. in $\mathbb{R}^{d}$, then $w=v p$-quasieverywhere in $\mathbb{R}^{d}$, see [15] for more details.

In 1997, Kinnunen proved that $\mathcal{M} f$ is $p$-quasicontinuous if $f \in W^{1, p}\left(\mathbb{R}^{d}\right)$ for any $1<p<\infty$. Motivated by Kinnunen's work [29], we shall prove the following result:

Theorem 1.5 (p-quasicontinuity). Let $1<p_{1}, \ldots, p_{m}<\infty$, and $1 / p=\sum_{i=1}^{m} 1 / p_{i}$. Suppose that $\vec{f}=\left(f_{1}, \ldots, f_{m}\right)$ with each $f_{i} \in W^{1, p_{i}}\left(\mathbb{R}^{d}\right)$, then $\mathscr{M}_{\mathcal{R}}(\vec{f})$ is p-quasicontinuous.

In 2010, Hajłasz and Malý [22] proved that $\mathcal{M} f$ is approximately differentiable a.e. provided that $f \in L^{1}\left(\mathbb{R}^{d}\right)$. Motivated by Hajłasz and Malý's work, we shall establish the following result:

Theorem 1.6. Let $\vec{f}=\left(f_{1}, \ldots, f_{m}\right)$ with each $f_{j} \in L^{1}\left(\mathbb{R}^{d}\right)$ being approximately differentiable a.e., then $\mathscr{M}_{\mathcal{R}}(\vec{f})$ is approximately differentiable a.e.

Remark 1.7. Since every function in $W^{1,1}\left(\mathbb{R}^{d}\right)$ space is approximately differentiable a.e., thus Theorem 1.6 yields that if each $f_{j} \in W^{1,1}\left(\mathbb{R}^{d}\right)$, then $\mathscr{M}_{\mathcal{R}}(\vec{f})$ is approximately differentiable a.e. However, it is unknown that whether $\mathscr{M}_{\mathcal{R}}(\vec{f})$ is weak differentiable when each $f_{j} \in W^{1,1}\left(\mathbb{R}^{d}\right)$, even in the case $m=1$ and $d \geq 2$.

As for the discrete type strong maximal functions, we have the following conclusion.

Theorem 1.7 (Properties of discrete strong maximal functions). Let $d \geq 1$ and $m \geq 2$. Then $\mathbb{M}_{\mathcal{R}}$ is bounded and continuous from $\ell^{1}\left(\mathbb{Z}^{d}\right) \times \cdots \times \ell^{1}\left(\mathbb{Z}^{d}\right)$ to $\mathrm{BV}\left(\mathbb{Z}^{d}\right)$. Equivalently, the operator $\vec{f} \mapsto \nabla \mathbb{M}_{\mathcal{R}}(\vec{f})$ is bounded and continuous from $\ell^{1}\left(\mathbb{Z}^{d}\right) \times \cdots \times \ell^{1}\left(\mathbb{Z}^{d}\right)$ to $\ell^{1}\left(\mathbb{Z}^{d}\right)$. Moreover, if $f_{j} \in \ell^{1}\left(\mathbb{Z}^{d}\right)$ for $1 \leq j \leq m$. Then

$$
\left\|\nabla \mathbb{M}_{\mathcal{R}}(\vec{f})\right\|_{\ell^{1}\left(\mathbb{Z}^{d}\right)} \lesssim_{d} \sum_{l=1}^{m}\left\|\nabla f_{l}\right\|_{\ell^{1}\left(\mathbb{Z}^{d}\right)} \prod_{j \neq l, 1 \leq j \leq m}\left\|f_{j}\right\|_{\ell^{1}\left(\mathbb{Z}^{d}\right)}
$$

Remark 1.8. we need to address the facts that: 
(i) $\mathbb{M}_{\mathcal{R}}$ is bounded and continuous from $W^{1, p_{1}}\left(\mathbb{Z}^{d}\right) \times \cdots \times W^{1, p_{m}}\left(\mathbb{Z}^{d}\right)$ to $W^{1, p}\left(\mathbb{Z}^{d}\right)$ for all $1<p_{1}, \ldots, p_{m}, p \leq \infty$ and $1 / p=\sum_{i=1}^{m} 1 / p_{i}$. This conclusion is basically implied by the following two facts. First, one can check that $\mathbb{M}_{\mathcal{R}}$ is bounded from $\ell^{p_{1}}\left(\mathbb{Z}^{d}\right) \times \cdots \times \ell^{p_{m}}\left(\mathbb{Z}^{d}\right)$ to $\ell^{p}\left(\mathbb{Z}^{d}\right)$. Secondly, it holds easily that $\left|\mathbb{M}_{\mathcal{R}}(\vec{f})-\mathbb{M}_{\mathcal{R}}(\vec{g})\right| \leq \sum_{\mu=1}^{m} \mathbb{M}_{\mathcal{R}}\left(\vec{F}_{\mu}\right)$, where $\vec{f}=\left(f_{1}, \ldots, f_{m}\right), \vec{g}=\left(g_{1}, \ldots, g_{m}\right)$ and $\vec{F}_{\mu}=\left(f_{1}, \ldots, f_{\mu-1}, f_{\mu}-g_{\mu}, g_{\mu+1}, \ldots, g_{m}\right)$. This together with (1.5) implies the continuity for $\mathbb{M}_{\mathcal{R}}$ from $W^{1, p_{1}}\left(\mathbb{Z}^{d}\right) \times \cdots \times W^{1, p_{m}}\left(\mathbb{Z}^{d}\right)$ to $W^{1, p}\left(\mathbb{Z}^{d}\right)$

(ii) When $d \geq 2$, the operator $f \mapsto \nabla M_{\mathcal{R}} f$ is bounded and continuous from $\ell^{1}\left(\mathbb{Z}^{d}\right)$ to $\ell^{p}\left(\mathbb{Z}^{d}\right)$ for $1<p \leq \infty$. However, the operator $f \mapsto \nabla M_{\mathcal{R}} f$ is not bounded from $\ell^{1}\left(\mathbb{Z}^{d}\right)$ to $\ell^{1}\left(\mathbb{Z}^{d}\right)$. This conclusions are basically implied by two facts. First, one can easily check that the operator $f \mapsto \nabla M_{\mathcal{R}} f$ is bounded and continuous from $\ell^{1}\left(\mathbb{Z}^{d}\right)$ to $\ell^{p}\left(\mathbb{Z}^{d}\right)$. Secondly, let $f(\vec{n})=\chi_{\{\overrightarrow{0}\}}(\vec{n})$. Note that $\|f\|_{\ell^{1}\left(\mathbb{Z}^{d}\right)}=1$ and $M_{\mathcal{R}} f(\vec{n})=\prod_{i=1}^{d}\left(\left|n_{i}\right|+1\right)^{-1}$ for each $\vec{n}=\left(n_{1}, \ldots, n_{d}\right) \in \mathbb{Z}^{d}$. It follows that $\left\|\nabla \mathbb{M}_{\mathcal{R}} f\right\|_{\ell^{1}\left(\mathbb{Z}^{d}\right)}=+\infty$. Thus, the operator $f \mapsto \nabla M_{\mathcal{R}} f$ is not bounded from $\ell^{1}\left(\mathbb{Z}^{d}\right)$ to $\ell^{1}\left(\mathbb{Z}^{d}\right) ;$

(iii) When $d \geq 2$, from Remark (ii) we know that the discrete strong maximal operator $M_{\mathcal{R}}$ is not bounded from $\ell^{1}\left(\mathbb{Z}^{d}\right)$ to $\mathrm{BV}\left(\mathbb{Z}^{d}\right)$. However, it was known that the discrete maximal operator $M$ is bounded from $\ell^{1}\left(\mathbb{Z}^{d}\right)$ to $\mathrm{BV}\left(\mathbb{Z}^{d}\right)$. Thus, the regularity property of discrete strong maximal operator $M_{\mathcal{R}}$ is worse than that of $M$ when $d \geq 2$;

(iv) The proof of Theorem A in [43] depends highly on a summability argument over the sequence of local maximal, local minimal of discrete multilinear maximal functions and the Brezis-Lieb lemma [8]. However, in the proof of Theorem 1.7, the above techniques are unnecessary and our proofs are more simple, direct and different than those in [43].

By (ii) of Remark 1.8, we can get the following result immediately.

Corollary 1.8. Let $d \geq 2$. Then the map $f \mapsto \nabla M_{\mathcal{R}} f$ is bounded from $\ell^{1}\left(\mathbb{Z}^{d}\right)$ to $\ell^{q}\left(\mathbb{Z}^{d}\right)$ if and only if $q>1$.

This paper will be organized as follows. Section 2 will be devoted to present the proof of Theorem 1.1. Section 3 will be devoted to give the proofs of Theorems 1.2 and 1.3. The proofs of Theorems 1.5 and 1.6 will be given in Sections 4 and 5, respectively. In Section 6, we shall prove Theorem 1.7. Finally, we introduce some properties of $u_{x, \vec{f}}$ in Section 7 . We would like to remark that the main ideas employed in the proofs of Theorems 1.1 and 1.7 are greatly motivated by [29, 46], but our methods and techniques are more delicate and complex than those in [29, 46]. It should be pointed out that the main ideas in the proofs of Theorems 1.2 and 1.3 are motivated by [44]. Our arguments in the proof of the bounded part in Theorem 1.7 are motivated by [10], but our methods and techniques are somewhat different and direct than those in [10]. In addition, the Brezis-Lieb lemma [8] is not necessary in the proof of the continuity part of Theorem 1.7.

Throughout this paper, if there exists a constant $c>0$ depending only on $\vartheta$ such that $A \leq c B$, we then write $A \lesssim_{\vartheta} B$ or $B \gtrsim_{\vartheta} A$; and if $A \lesssim_{\vartheta} B \lesssim_{\vartheta} A$, we then write $A \sim_{\vartheta} B$.

\section{Properties on Sobolev Spaces}

2.1. Prelimary lemmas. We first present several preliminary lemmas, which play important roles in the proof of Theorem 1.1. Some basic ideas will be taken from [46], where the proof for the continuity in $W^{1, p}\left(\mathbb{R}^{d}\right)$ of the Hardy-Littlewood maximal operator has been given. We only consider the case $d=2$ and other cases are analogous and more complex. 
For $A \subset \mathbb{R}^{2}$ and $x \in \mathbb{R}^{2}$, define

$$
d(x, A):=\inf _{a \in A}|x-a| \text { and } A_{(\lambda)}:=\left\{x \in \mathbb{R}^{2} ; d(x, A) \leq \lambda\right\} \text { for } \lambda \geq 0 .
$$

We denote by $\|f\|_{p, A}$ the $L^{p}$-norm of $f \chi_{A}$ for all measurable sets $A \subset \mathbb{R}^{2}$. Let $1 / p=\sum_{j=1}^{m} 1 / p_{j}$ and $1<p_{1}, p_{2}, \ldots, p_{m}, p<\infty$. Let $\vec{f}=\left(f_{1}, \ldots, f_{m}\right)$ with each $f_{j} \in L^{p_{j}}\left(\mathbb{R}^{2}\right)$. For convenience, we set $\mathbb{R}_{+}=(0, \infty)$ and $\overline{\mathbb{R}}_{+}=[0, \infty)$. We also set

$$
\begin{aligned}
&\left(\overline{\mathbb{R}}_{+}\right)_{1}^{4}=\left\{\left(r_{1}, r_{2}, 0,0\right):\left(r_{1}, r_{2}\right) \in \overline{\mathbb{R}}_{+}^{2}, r_{1}+r_{2}>0\right\}, \\
&\left(\overline{\mathbb{R}}_{+}\right)_{2}^{4}=\left\{\left(0,0, r_{3}, r_{4}\right):\left(r_{3}, r_{4}\right) \in \overline{\mathbb{R}}_{+}^{2}, r_{3}+r_{4}>0\right\}, \\
&\left(\overline{\mathbb{R}}_{+}\right)_{1,2}^{4}=\left\{\left(r_{1}, r_{2}, r_{3}, r_{4}\right):\left(r_{1}, r_{2}, r_{3}, r_{4}\right) \in \overline{\mathbb{R}}_{+}^{4}, r_{1}+r_{2}>0, r_{3}+r_{4}>0\right\} .
\end{aligned}
$$

Define the function $u_{\left(x_{1}, x_{2}\right), \vec{f}}: \overline{\mathbb{R}}_{+}^{4} \rightarrow \mathbb{R}$ by

$$
\begin{array}{r}
u_{\left(x_{1}, x_{2}\right), \vec{f}}\left(r_{1,1}, r_{1,2}, 0,0\right):=\frac{1}{\left(r_{1,1}+r_{1,2}\right)^{m}} \prod_{j=1}^{m} \int_{x_{1}-r_{1,1}}^{x_{1}+r_{1,2}}\left|f_{j}\left(y_{1}, x_{2}\right)\right| d y_{1} \quad \text { for }\left(r_{1,1}, r_{1,2}, 0,0\right) \in\left(\overline{\mathbb{R}}_{+}\right)_{1}^{4} ; \\
u_{\left(x_{1}, x_{2}\right), \vec{f}}\left(0,0, r_{2,1}, r_{2,2}\right):=\frac{1}{\left(r_{2,1}+r_{2,2}\right)^{m}} \prod_{j=1}^{m} \int_{x_{2}-r_{2,1}}^{x_{2}+r_{2,2}}\left|f_{j}\left(x_{1}, y_{2}\right)\right| d y_{2} \quad \text { for }\left(0,0, r_{2,1}, r_{2,2}\right) \in\left(\overline{\mathbb{R}}_{+}\right)_{2}^{4} ; \\
u_{\left(x_{1}, x_{2}\right), \vec{f}}\left(r_{1,1}, r_{1,2}, r_{2,1}, r_{2,2}\right):=\prod_{i=1}^{2} \frac{1}{\left(r_{i, 1}+r_{i, 2}\right)^{m}} \prod_{j=1}^{m} \int_{x_{1}-r_{1,1}}^{x_{1}+r_{1,2}} \int_{x_{2}-r_{2,1}}^{x_{2}+r_{2,2}}\left|f_{j}\left(y_{1}, y_{2}\right)\right| d y_{1} d y_{2}, \\
\text { for }\left(r_{1,1}, r_{1,2}, r_{2,1}, r_{2,2}\right) \in\left(\overline{\mathbb{R}}_{+}\right)_{1,2}^{4} .
\end{array}
$$

In particular, we denote $u_{\left(x_{1}, x_{2}\right), \vec{f}}(0,0,0,0)=\prod_{j=1}^{m}\left|f_{j}\left(x_{1}, x_{2}\right)\right|$. We can write

$$
\mathscr{M}_{\mathcal{R}}(\vec{f})(x)=\sup _{r_{1,1}, r_{1,2}, r_{2,1}, r_{2,2}>0} u_{\left(x_{1}, x_{2}\right), \vec{f}}\left(r_{1,1}, r_{1,2}, r_{2,1}, r_{2,2}\right) .
$$

For a fixed point $x=\left(x_{1}, x_{2}\right) \in \mathbb{R}^{2}$, we define the sets $\mathcal{B}_{i}(\vec{f})\left(x_{1}, x_{2}\right)(i=1,2,3)$ by

$$
\begin{aligned}
& \mathcal{B}_{1}(\vec{f})\left(x_{1}, x_{2}\right):=\left\{\left(r_{1,1}, r_{1,2}, r_{2,1}, r_{2,2}\right) \in \overline{\mathbb{R}}_{+}^{4}: \mathscr{M}_{\mathcal{R}}(\vec{f})\left(x_{1}, x_{2}\right)=\right. \\
& \limsup _{\substack{\left(r_{1,1, k}, r_{1,2, k}, r_{2,1, k}, r_{2,2, k}\right) \\
\rightarrow\left(r_{1,1}, r_{1,2}, r_{2,1}, r_{2,2}\right)}} u_{\left(x_{1}, x_{2}\right), \vec{f}}\left(r_{1,1, k}, r_{1,2, k}, r_{2,1, k}, r_{2,2, k}\right) \\
& \text { for some } \left.r_{1,1, k}, r_{1,2, k}, r_{2,1, k}, r_{2,2, k}>0\right\} \text {. } \\
& \mathcal{B}_{2}(\vec{f})\left(x_{1}, x_{2}\right):=\left\{\left(r_{1,1}, r_{1,2}, r_{2,1}, r_{2,2}\right) \in \overline{\mathbb{R}}_{+}^{2} \times\{(0,0)\}: \mathscr{M}_{\mathcal{R}}(\vec{f})\left(x_{1}, x_{2}\right)=\right. \\
& \left.\limsup _{\left(r_{1,1, k}, r_{1,2, k}\right) \rightarrow\left(r_{1,1}, r_{1,2}\right)} u_{\left(x_{1}, x_{2}\right), \vec{f}}\left(r_{1,1, k}, r_{1,2, k}, 0,0\right) \text { for some } r_{1,1, k}, r_{1,2, k}>0\right\} . \\
& \mathcal{B}_{3}(\vec{f})\left(x_{1}, x_{2}\right):=\left\{\left(r_{1}, r_{2}\right) \in\{(0,0)\} \times \overline{\mathbb{R}}_{+}^{2}: \mathscr{M}_{\mathcal{R}}(\vec{f})\left(x_{1}, x_{2}\right)=\right. \\
& \left.\limsup _{\left., r_{2,2, k}\right) \rightarrow\left(r_{2,1}, r_{2,2}\right)} u_{\left(x_{1}, x_{2}\right), \vec{f}}\left(0,0, r_{2,1, k}, r_{2,2, k}\right) \text { for some } r_{2,1, k}, r_{2,2, k}>0\right\} .
\end{aligned}
$$

The function $u_{\left(x_{1}, x_{2}\right), \vec{f}}$ enjoys the following properties: 
Lemma 2.1. Let $\vec{f}=\left(f_{1}, \ldots, f_{m}\right)$ with each $f_{j} \in L^{p_{j}}\left(\mathbb{R}^{2}\right)$ for $1<p_{j}<\infty,(j=1,2, \ldots, m)$. Then the following statements hold:

(i) $u_{\left(x_{1}, x_{2}\right), \vec{f}}$ are continuous on $\left(\overline{\mathbb{R}}_{+}\right)_{+}^{4}:=\left\{\left(r_{1,1}, r_{1,2}, r_{2,1}, r_{2,2}\right) \in \overline{\mathbb{R}}_{+}^{4}: r_{1,1}+r_{1,2}, r_{2,1}+r_{2,2}>\right.$ $0\}$ for all $\left(x_{1}, x_{2}\right) \in \mathbb{R}^{2}$, and continuous on $\overline{\mathbb{R}}_{+}^{4}$ for a.e. $\left(x_{1}, x_{2}\right) \in \mathbb{R}^{2}$;

$$
\lim _{\substack{\left(r_{1,1}, r_{1,2}, r_{2,1}, r_{2,2}\right) \in \overline{\mathbb{R}}_{+}^{4} \\ r_{1,1}+r_{1,2}, r_{2,1}+r_{2,2} \rightarrow \infty}} u_{\left(x_{1}, x_{2}\right), \vec{f}}\left(r_{1,1}, r_{1,2}, r_{2,1}, r_{2,2}\right)=0, \quad \text { for a.e. }\left(x_{1}, x_{2}\right) \in \mathbb{R}^{2} ;
$$

$\mathcal{B}_{1}(\vec{f})\left(x_{1}, x_{2}\right)$ are nonempty and closed for every $\left(x_{1}, x_{2}\right) \in \mathbb{R}^{2}$;

(ii) $u_{\left(x_{1}, x_{2}\right), \vec{f}}$ are continuous on $\left\{\left(r_{1,1}, r_{1,2}\right) \in \overline{\mathbb{R}}_{+}^{2}: r_{1,1}+r_{1,2}>0\right\} \times\{(0,0)\}$ for all $x_{1} \in \mathbb{R}$ and a.e. $x_{2} \in \mathbb{R}$, and continuous at $(0,0,0,0)$ for a.e. $\left(x_{1}, x_{2}\right) \in \mathbb{R}^{2}$;

$$
\lim _{\substack{\left(r_{1,1}, r_{1,2}\right) \in \mathbb{\mathbb { R }}_{+}^{2} \\ r_{1,1}+r_{1,2} \rightarrow \infty}} u_{\left(x_{1}, x_{2}\right), \vec{f}}\left(r_{1,1}, r_{1,2}, 0,0\right)=0, \quad \text { for all } x_{1} \in \mathbb{R} \quad \text { and a.e. } \quad x_{2} \in \mathbb{R} ;
$$

$\mathcal{B}_{2}(\vec{f})\left(x_{1}, x_{2}\right)$ are nonempty and closed for a.e. $\left(x_{1}, x_{2}\right) \in \mathbb{R}^{2}$;

(iii) $u_{\left(x_{1}, x_{2}\right), \vec{f}}$ are continuous on $\{(0,0)\} \times\left\{\left(r_{2,1}, r_{2,2}\right) \in \overline{\mathbb{R}}_{+}^{2}: r_{2,1}+r_{2,2}>0\right\}$ for all $x_{2} \in \mathbb{R}$ and a.e. $x_{1} \in \mathbb{R}$ and continuous at $(0,0,0,0)$ for a.e. $\left(x_{1}, x_{2}\right) \in \mathbb{R}^{2}$;

$$
\lim _{\substack{\left(r_{2,1}, r_{2,2}\right) \in \overline{\mathbb{R}}_{+}^{2} \\ r_{2,1}+r_{2,2} \rightarrow \infty}} u_{\left(x_{1}, x_{2}\right), \vec{f}}\left(0,0, r_{2,1}, r_{2,2}\right)=0, \quad \text { for all } x_{2} \in \mathbb{R} \quad \text { and a.e. } \quad x_{1} \in \mathbb{R} ;
$$

$\mathcal{B}_{3}(\vec{f})\left(x_{1}, x_{2}\right)$ are nonempty and closed for a.e. $\left(x_{1}, x_{2}\right) \in \mathbb{R}^{2}$.

Proof. (i) The first statement follows from the integrability of $f_{j}$. The proof of the continuity on $\overline{\mathbb{R}}_{+}^{4}$ for a.e. $\left(x_{1}, x_{2}\right) \in \mathbb{R}^{2}$ is very delicate. So, we shall prove it in the last section. We can see easily that for any $\left(x_{1}, x_{2}\right) \in \mathbb{R}^{2}$, it holds that

$$
\lim _{\substack{\left(r_{1,1}, r_{1,2}, r_{2,1}, r_{2,2}\right) \in \overline{\mathbb{R}}_{+}^{4} \\ r_{1,1}+r_{1,2}, r_{2,1}+r_{2,2} \rightarrow \infty}} u_{\left(x_{1}, x_{2}\right), \vec{f}}\left(r_{1,1}, r_{1,2}, r_{2,1}, r_{2,2}\right)=0,
$$

since $u_{\left(x_{1}, x_{2}\right), f}\left(r_{1,1}, r_{1,2}, r_{2,1}, r_{2,2}\right) \leq\left|\left(r_{1,1}+r_{1,2}\right)\left(r_{2,1}+r_{2,2}\right)\right|^{-1 / p} \prod_{j=1}^{m}\left\|f_{j}\right\|_{L^{p_{j}\left(\mathbb{R}^{2}\right)}}$. But, when $0<r_{1,1}+r_{1,2}+r_{2,1}+r_{2,2} \rightarrow \infty$, we should treat more carefully, and we shall prove it in the last section. The last statement can be checked easily.

(ii) The first statement follows from the integrability of $f_{j}$. The continuity at $(0,0,0,0)$ will be checked in the last section. Since $u_{\left(x_{1}, x_{2}\right), f}(\vec{r}) \leq\left|r_{1,1}+r_{1,2}\right|^{-1 / p} \prod_{j=1}^{m}\left\|f_{j}\left(\cdot, x_{2}\right)\right\|_{L^{p_{j}(\mathbb{R})}}$ for any $\left(r_{1,1}, r_{1,2}, 0,0\right) \in \mathbb{R}_{+} \times\{(0,0)\}$ and all $x_{1} \in \mathbb{R}$ and a.e. $x_{2} \in \mathbb{R}$, we get

$$
\lim _{\substack{\left(r_{1,1}, r_{1,2}\right) \in \overline{\mathbb{R}}_{+}^{2} \\ r_{1,1}+r_{1,2} \rightarrow \infty}} u_{\left(x_{1}, x_{2}\right), \vec{f}}\left(r_{1,1}, r_{1,2}, 0,0\right)=0 .
$$

The last statement can be checked easily.

(iii) (iii) is the same as in (ii).

Lemma 2.2. The following relationships between $\mathscr{M}_{\mathcal{R}}(\vec{f})$ and $u_{\left(x_{1}, x_{2}\right), \vec{f}}$ are valid.

(iv) $\mathscr{M}_{\mathcal{R}}(\vec{f})\left(x_{1}, x_{2}\right)=\prod_{j=1}^{m}\left|f_{j}\left(x_{1}, x_{2}\right)\right|$ for a.e. $\left(x_{1}, x_{2}\right) \in \mathbb{R}^{2}$ such that $\overrightarrow{0} \in \bigcup_{i=1}^{3} \mathcal{B}_{i}(f)\left(x_{1}, x_{2}\right)$;

(v) $\mathscr{M}_{\mathcal{R}}(\vec{f})\left(x_{1}, x_{2}\right)=u_{\left(x_{1}, x_{2}\right), \vec{f}}(\vec{r})$ for a.e. $\left(x_{1}, x_{2}\right) \in \mathbb{R}^{2}$ such that $\vec{r} \in \mathcal{B}_{1}(\vec{f})\left(x_{1}, x_{2}\right) \cap\left(\overline{\mathbb{R}}_{+}\right)_{1}^{4}$;

(vi) $\mathscr{M}_{\mathcal{R}}(\vec{f})\left(x_{1}, x_{2}\right)=u_{\left(x_{1}, x_{2}\right), \vec{f}}(\vec{r})$ for a.e. $\left(x_{1}, x_{2}\right) \in \mathbb{R}^{2}$ such that $\vec{r} \in \mathcal{B}_{1}(\vec{f})\left(x_{1}, x_{2}\right) \cap\left(\overline{\mathbb{R}}_{+}\right)_{2}^{4}$; 
(vii) $\mathscr{M}_{\mathcal{R}}(\vec{f})\left(x_{1}, x_{2}\right)=u_{\left(x_{1}, x_{2}\right), \vec{f}}(\vec{r})$ for a.e. $\left(x_{1}, x_{2}\right) \in \mathbb{R}^{2}$ such that $\vec{r} \in \mathcal{B}_{2}(\vec{f})\left(x_{1}, x_{2}\right)$;

(viii) $\mathscr{M}_{\mathcal{R}}(\vec{f})\left(x_{1}, x_{2}\right)=u_{\left(x_{1}, x_{2}\right), \vec{f}}(\vec{r})$ for a.e. $\left(x_{1}, x_{2}\right) \in \mathbb{R}^{2}$ such that $\vec{r} \in \mathcal{B}_{3}(\vec{f})\left(x_{1}, x_{2}\right)$;

(ix) $\mathscr{M}_{\mathcal{R}}(\vec{f})\left(x_{1}, x_{2}\right)=u_{\left(x_{1}, x_{2}\right), \vec{f}}(\vec{r})$ if $\vec{r} \in \mathcal{B}_{1}(\vec{f})(x) \cap\left(\overline{\mathbb{R}}_{+}\right)_{1,2}^{4}$ for all $x \in \mathbb{R}^{2}$.

For convenience, for any $\vec{r}=\left(r_{1}, r_{2}, \ldots, r_{d}\right) \in \mathbb{R}_{+}^{d}$ and $x=\left(x_{1}, x_{2}, \ldots, x_{d}\right) \in \mathbb{R}^{d}$, we let

$$
R_{\vec{r}}(x)=\left\{y=\left(y_{1}, y_{2}, \ldots, y_{d}\right) \in \mathbb{R}^{d} ;\left|y_{i}-x_{i}\right|<r_{i}, i=1,2, \ldots, d\right\} .
$$

Lemma 2.3. Let $\Lambda>0, \vec{\Lambda}=(\Lambda, \Lambda)$ and $\overrightarrow{0}=(0,0), 1<p, p_{i}<\infty$ and $1 / p=\sum_{i=1}^{m} 1 / p_{i}$, and $\vec{f}=\left(f_{1}, \ldots, f_{m}\right)$ with each $f_{i} \in L^{p_{i}}\left(\mathbb{R}^{2}\right)$. Let $\vec{f}_{j}=\left(f_{1, j}, \ldots, f_{m, j}\right)$ such that $f_{i, j} \rightarrow f_{i}$ in $L^{p_{i}}\left(\mathbb{R}^{2}\right)$ when $j \rightarrow \infty$ for all $i=1,2, \ldots, m$. Then for all $\lambda>0$ and $i=1,2,3$, we have

$$
\lim _{j \rightarrow \infty}\left|\left\{x \in R_{\vec{\Lambda}}(\overrightarrow{0}) ; \mathcal{B}_{i}\left(\vec{f}_{j}\right)(x) \not \mathcal{B}_{i}(\vec{f})(x)_{(\lambda)}\right\}\right|=0 .
$$

Proof. Without loss of generality we may assume all $f_{i, j} \geq 0$ and $f_{i} \geq 0$. We shall prove (2.1) for the case $i=1$ and the other cases are analogous. Let $\lambda>0$ and $\Lambda>0$. We first conclude that the set $\left\{x \in \mathbb{R}^{2} ; \mathcal{B}_{1}\left(\overrightarrow{f_{j}}\right)(x) \nsubseteq \mathcal{B}_{1}(\vec{f})(x)_{(\lambda)}\right\}$ is measurable for all $j \geq 1$. To see this, let $E$ be the set of all points which are not Lebesgue points of any of the functions $f_{i, j}$ and $f_{i}$. Obviously, $|E|=0$. We denote by $\mathbb{Q}_{+}$the set of positive rationals. Fix $j \geq 1$, we can write

$$
\begin{aligned}
& \left\{x \in \mathbb{R}^{2} \backslash E: \mathcal{B}_{1}\left(\overrightarrow{f_{j}}\right)(x) \nsubseteq \mathcal{B}_{1}(\vec{f})(x)_{\lambda}\right\} \\
& =\bigcup_{i=1}^{\infty} \bigcap_{k=1}^{\infty}\left\{x \in \mathbb{R}^{2}: \exists \vec{r} \in \mathbb{R}_{+}^{4} \text { s.t. } d\left(\vec{r}, \mathcal{B}_{1}(\vec{f})(x)\right)>\lambda+\frac{1}{i}\right. \\
& \left.\quad \text { and } \mathscr{M}_{\mathcal{R}}\left(\vec{f}_{j}\right)(x)<u_{x, \vec{f}_{j}}(\vec{r})+\frac{1}{k}\right\} \\
& =\bigcup_{i=1}^{\infty} \bigcap_{k=1}^{\infty} \bigcup_{\vec{t} \in \mathbb{Q}_{+}^{4}}\left(\left\{x \in \mathbb{R}^{2}: d\left(\vec{t}, \mathcal{B}_{1}(\vec{f})(x)\right)>\lambda+\frac{1}{i}\right\} \bigcap\right. \\
& \left.\quad\left\{x \in \mathbb{R}^{2}: \mathscr{M}_{\mathcal{R}}\left(\vec{f}_{j}\right)(x)<u_{x, \vec{f}_{j}}(\vec{t})+\frac{1}{k}\right\}\right) .
\end{aligned}
$$

On the other hand, for any fixed $\vec{t} \in \mathbb{Q}_{+}^{4}$, we have

$$
\left\{x: d\left(\vec{t}, \mathcal{B}_{1}(\vec{f})(x)\right)>\lambda\right\}=\bigcup_{l=1}^{\infty} \bigcap_{\vec{s} \in \mathbb{Q}_{+}^{4} \cap\{\vec{s}:|\vec{s}-\vec{t}| \leq \lambda\}}\left\{x \in \mathbb{R}^{2}: \mathscr{M}_{\mathcal{R}}(\vec{f})(x)>u_{x, \vec{f}}(\vec{s})+\frac{1}{l}\right\} .
$$

Therefore, we get the measurability of $\left\{x \in \mathbb{R}^{2} ; \mathcal{B}_{1}\left(\overrightarrow{f_{j}}\right)(x) \nsubseteq \mathcal{B}_{1}(\vec{f})(x)_{(\lambda)}\right\}$ for any $j \geq 1$.

Now, we claim that for a.e. $x \in R_{\vec{\Lambda}}(\overrightarrow{0})$, there exists $\gamma(x) \in \mathbb{N} \backslash\{0\}$ such that

$$
u_{x, \vec{f}}(\vec{r})<\mathscr{M}_{\mathcal{R}}(\vec{f})(x)-\frac{1}{\gamma(x)}, \text { when } d\left(\vec{r}, \mathcal{B}_{1}(\vec{f})(x)\right)>\lambda \text {. }
$$

Actually, if (2.2) does not hold, then for a.e. $x \in R_{\vec{\Lambda}}(\overrightarrow{0})$, there exists a bounded sequence of $\left\{\overrightarrow{r_{k}}\right\}_{k=1}^{\infty}$ such that

$$
\lim _{k \rightarrow \infty} u_{x, \vec{f}}\left(\overrightarrow{r_{k}}\right)=\mathscr{M}_{\mathcal{R}}(\vec{f})(x) \text { and } d\left(\overrightarrow{r_{k}}, \mathcal{B}_{1}(\vec{f})(x)\right)>\lambda .
$$

Hence, we may choose a subsequence $\left\{\overrightarrow{s_{k}}\right\}_{k=1}^{\infty}$ of $\left\{\overrightarrow{r_{k}}\right\}_{k=1}^{\infty}$ such that $\overrightarrow{s_{k}} \rightarrow \vec{r}$ as $k \rightarrow \infty$. It follows that $\vec{r} \in \mathcal{B}_{1}(\vec{f})(x)$ and $d\left(\vec{r}, \mathcal{B}_{1}(\vec{f})(x)\right) \geq \lambda$, which is a contradiction. Therefore, (2.2) holds. Let

$$
\begin{aligned}
& A_{1, j}:=\left\{x \in \mathbb{R}^{2}:\left|\mathscr{M}_{\mathcal{R}}\left(\overrightarrow{f_{j}}\right)(x)-\mathscr{M}_{\mathcal{R}}(\vec{f})(x)\right| \geq(4 \gamma)^{-1}\right\}, \\
& A_{2, j}:=\left\{x \in \mathbb{R}^{2}:\left|u_{x, \vec{f}_{j}}(\vec{r})-u_{x, \vec{f}}(\vec{r})\right| \geq(2 \gamma)^{-1} \text { if } d\left(\vec{r}, \mathcal{B}_{1}(\vec{f})(x)\right)>\lambda\right\},
\end{aligned}
$$




$$
A_{3, j}:=\left\{x \in \mathbb{R}^{2}: u_{x, \vec{f}_{j}}(\vec{r})<\mathscr{M}_{\mathcal{R}}\left(\vec{f}_{j}\right)(x)-(4 \gamma)^{-1}, \text { if } d\left(\vec{r}, \mathcal{B}_{1}(\vec{f})(x)\right)>\lambda\right\} .
$$

Given $\epsilon \in(0,1)$, we get from (2.2) that there exists $\gamma=\gamma(\Lambda, \lambda, \epsilon) \in \mathbb{N} \backslash\{0\}$ and a measurable set $E_{0}$ with $\left|E_{0}\right|<\epsilon$ such that

$$
\begin{aligned}
R_{\vec{\Lambda}}(\overrightarrow{0}) & \subset\left\{x \in \mathbb{R}^{2}: u_{x, \vec{f}}(\vec{r})<\mathscr{M}_{\mathcal{R}}(\vec{f})(x)-\gamma^{-1}, \text { if } d\left(\vec{r}, \mathcal{B}_{1}(\vec{f})(x)\right)>\lambda\right\} \cup E_{0} \\
& \subset A_{1, j} \cup A_{2, j} \cup A_{3, j} \cup E_{0} .
\end{aligned}
$$

Let $\bar{A}$ be the set of all points $x$ such that $x$ is a Lebesgue point of all $f_{j}$. Note that $\left|\mathbb{R}^{2} \backslash \bar{A}\right|=0$. One can easily check that $A_{3, j} \cap \bar{A} \subset\left\{x \in \mathbb{R}^{2}: \mathcal{B}_{1}\left(\overrightarrow{f_{j}}\right)(x) \subset \mathcal{B}_{1}(\vec{f})(x)_{(\lambda)}\right\}$. This together with (2.3) yields that

$$
\left\{x \in R_{\vec{\Lambda}}(\overrightarrow{0}) ; \mathcal{B}_{1}\left(\vec{f}_{j}\right)(x) \nsubseteq \mathcal{B}_{1}(\vec{f})(x)_{(\lambda)}\right\} \subset A_{1, j} \cup A_{2, j} \cup E_{0} \cup\left(\mathbb{R}^{2} \backslash \bar{A}\right) .
$$

Since $f_{i, j} \rightarrow f_{i}$ in $L^{p_{i}}\left(\mathbb{R}^{2}\right)$ when $j \rightarrow \infty$, then there exists $N_{i}=N_{i}(\epsilon, \gamma) \in \mathbb{N}$ such that

$$
\left\|f_{i, j}-f_{i}\right\|_{L^{p_{i}\left(\mathbb{R}^{2}\right)}}<\frac{\epsilon}{\gamma} \text { and }\left\|f_{i, j}\right\|_{L^{p_{i}\left(\mathbb{R}^{2}\right)}} \leq\left\|f_{i}\right\|_{L^{p_{i}\left(\mathbb{R}^{2}\right)}}+1 \quad \forall j \geq N_{i} .
$$

Moreover, it holds that

$$
\begin{aligned}
& \left|\mathscr{M}_{\mathcal{R}}\left(\vec{f}_{j}\right)(x)-\mathscr{M}_{\mathcal{R}}(\vec{f})(x)\right| \\
& \leq \sup _{\substack{R \ni \mathcal{R} \\
R \in \mathcal{R}}} \frac{1}{|R|^{m}}\left|\prod_{i=1}^{m} \int_{R} f_{i, j}(y) d y-\prod_{i=1}^{m} \int_{R} f_{i}(y) d y\right| \\
& \leq \sum_{l=1}^{m} \sup _{\substack{R \ni x \\
R \in \mathcal{R}}} \frac{1}{|R|^{m}} \prod_{\mu=1}^{l-1} \int_{R} f_{\mu}(y) d y \prod_{\nu=l+1}^{m} \int_{R} f_{\nu, j}(y) d y \int_{R}\left|f_{l, j}(y)-f_{l}(y)\right| d y \\
& \leq \sum_{l=1}^{m} \mathscr{M}_{\mathcal{R}}\left(\vec{F}_{j}^{l}\right)(x),
\end{aligned}
$$

where $\vec{F}_{j}^{l}=\left(f_{1}, \ldots, f_{l-1}, f_{l, j}-f_{l}, f_{l+1, j}, \ldots, f_{m, j}\right)$. Let $N_{0}=\max _{1 \leq j \leq m} N_{j}$. Then, for any $j \geq N_{0}$, we get from (2.5) and (2.6) that

$$
\begin{aligned}
\left|A_{1, j}\right| & \leq(4 \gamma)^{p}\left\|\mathscr{M}_{\mathcal{R}}\left(\vec{f}_{j}\right)-\mathscr{M}_{\mathcal{R}}(\vec{f})\right\|_{L^{p}\left(\mathbb{R}^{2}\right)}^{p} \\
& \leq(4 \gamma m)^{p} \sum_{l=1}^{m} \prod_{\mu=1}^{l-1}\left\|f_{\mu}\right\|_{L^{p_{\mu}\left(\mathbb{R}^{2}\right)}}^{p} \prod_{\nu=l+1}^{m}\left\|f_{\nu, j}\right\|_{L^{p_{\nu}\left(\mathbb{R}^{d}\right)}}^{p}\left\|f_{l, j}-f_{l}\right\|_{L^{p_{l}\left(\mathbb{R}^{2}\right)}}^{p} \\
& \lesssim_{m, p_{1}, \ldots, p_{m}, p} \epsilon .
\end{aligned}
$$

Since

$$
\left|u_{x, \vec{f}_{j}}(\vec{r})-u_{x, \vec{f}}(\vec{r})\right| \leq \sum_{l=1}^{m} \mathscr{M}_{\mathcal{R}}\left(\vec{F}_{j}^{l}\right)(x) .
$$

Similarly, $\left|A_{2, j}\right| \lesssim_{m, p_{1}, \ldots, p_{m}, p} \epsilon$ for any $j \geq N_{0}$. This together with (2.4) and (2.7) yields (2.1).

For any fixed $h>0$ and $f_{i} \in L^{p_{i}}\left(\mathbb{R}^{2}\right)$ with $1<p_{i}<\infty$, define

$$
\left(f_{i}\right)_{h}^{l}(x)=\frac{\left(f_{i}\right)_{\tau(h)}^{l}(x)-f_{i}(x)}{h} \text { and }\left(f_{i}\right)_{\tau(h)}^{l}(x)=f_{i}\left(x+h \vec{e}_{l}\right), \quad l=1,2 .
$$

It is well known that for $l=1,2$ and $1<p_{i}<\infty,\left(f_{i}\right)_{\tau(h)}^{l} \rightarrow f_{i}$ in $L^{p_{i}}\left(\mathbb{R}^{2}\right)$ when $h \rightarrow 0$, and if $f_{i} \in W^{1, p_{i}}\left(\mathbb{R}^{2}\right)$ we have $\left(f_{i}\right)_{h}^{l} \rightarrow D_{l} f_{i}$ in $L^{p_{i}}\left(\mathbb{R}^{2}\right)$ when $h \rightarrow 0$ (see [18]). Let $A, B$ be two subsets 
of $\mathbb{R}^{2}$, we define the Hausdorff distance of $A$ and $B$ by

$$
\pi(A, B):=\inf \left\{\delta>0: A \subset B_{(\delta)} \text { and } B \subset A_{(\delta)}\right\} .
$$

Applying Lemma 2.3, we can get the following corollary.

Corollary 2.4. Let $f_{i} \in L^{p_{i}}\left(\mathbb{R}^{2}\right)$ with $1<p_{i}<\infty$. Then for all $\Lambda>0, \lambda>0, i=1,2,3$ and $l=1,2$, we have

$$
\lim _{h \rightarrow 0}\left|\left\{x \in R_{\vec{\Lambda}}(\overrightarrow{0}) ; \pi\left(\mathcal{B}_{i}(\vec{f})(x), \mathcal{B}_{i}(\vec{f})\left(x+h \vec{e}_{l}\right)\right)>\lambda\right\}\right|=0 .
$$

Proof. Fix $i \in\{1,2,3\}$. It suffices to show that

$$
\lim _{h \rightarrow 0} \mid\left\{x \in R_{\vec{\Lambda}}(\overrightarrow{0}): \mathcal{B}_{i}(\vec{f})(x) \nsubseteq \mathcal{B}_{i}(\vec{f})\left(x+h \vec{e}_{l}\right)_{(\lambda)} \text { or } \mathcal{B}_{i}(\vec{f})\left(x+h \vec{e}_{l}\right) \nsubseteq \mathcal{B}_{i}(\vec{f})(x)_{(\lambda)}\right\} \mid=0 .
$$

One can easily check that

$$
\mathcal{B}_{i}(\vec{f})\left(x+h \vec{e}_{l}\right)=\mathcal{B}_{i}\left(\vec{f}_{\tau(h)}^{l}\right)(x) \text { and } \mathcal{B}_{i}(\vec{f})(x)=\mathcal{B}_{i}\left(\vec{f}_{\tau(-h)}^{l}\right)\left(x+h \vec{e}_{l}\right) .
$$

Here $\vec{f}_{\tau(h)}^{\vec{b}}=\left(f_{1}\left(x+h \vec{e}_{l}\right), \ldots, f_{m}\left(x+h \vec{e}_{l}\right)\right)$. It follows that

$$
\begin{aligned}
& \left\{x \in R_{\vec{\Lambda}}(\overrightarrow{0}): \mathcal{B}_{i}(\vec{f})(x) \nsubseteq \mathcal{B}_{i}(\vec{f})\left(x+h \vec{e}_{l}\right)_{(\lambda)}\right\} \\
& =\left\{x \in R_{\vec{\Lambda}}(\overrightarrow{0}): \mathcal{B}_{i}\left(\vec{f}_{\tau(-h)}^{l}\right)\left(x+h \vec{e}_{l}\right) \nsubseteq \mathcal{B}_{i}(\vec{f})\left(x+h \vec{e}_{l}\right)_{(\lambda)}\right\} \\
& \subset\left\{x \in R_{\Lambda \overrightarrow{+} 1}(\overrightarrow{0}): \mathcal{B}_{i}\left(\vec{f}_{\tau(-h)}^{l}\right)(x) \nsubseteq \mathcal{B}_{i}(\vec{f})(x)_{(\lambda)}\right\}-h \vec{e}_{l}
\end{aligned}
$$

where $\Lambda \overrightarrow{+} 1=(\Lambda+1, \Lambda+1)$ and $|h| \leq 1$. Moreover,

$$
\left\{x \in R_{\vec{\Lambda}}(\overrightarrow{0}): \mathcal{B}_{i}(\vec{f})\left(x+h e_{l}\right) \nsubseteq \mathcal{B}_{i}(\vec{f})(x)_{(\lambda)}\right\}=\left\{x \in R_{\vec{\Lambda}}(\overrightarrow{0}): \mathcal{B}_{i}\left(\vec{f}_{\tau(h)}^{t}\right)(x)_{(\lambda)} \nsubseteq \mathcal{B}_{i}(\vec{f})(x)_{(\lambda)}\right\} .
$$

We note that $\left(f_{i}\right)_{\tau(h)}^{l} \rightarrow f_{i}$ in $L^{p_{i}}\left(\mathbb{R}^{2}\right)$ when $h \rightarrow 0$. By Lemma 2.3, it yields that

$$
\lim _{h \rightarrow 0}\left|\left\{x \in R_{\Lambda \overrightarrow{+} 1}(\overrightarrow{0}): \mathcal{B}_{i}\left(\vec{f}_{\tau(-h)}^{l}\right)(x) \nsubseteq \mathcal{B}_{i}(\vec{f})(x)_{(\lambda)}\right\}\right|=0
$$

and

$$
\lim _{h \rightarrow 0}\left|\left\{x \in R_{\vec{\Lambda}}(\overrightarrow{0}): \mathcal{B}_{i}\left(\vec{f}_{\tau(h)}^{l}\right)(x)_{(\lambda)} \nsubseteq \mathcal{B}_{i}(\vec{f})(x)_{(\lambda)}\right\}\right|=0 .
$$

Now, it is easy to see that (2.8) follows from (2.9) $-(2.12)$.

We now state some formulas for the derivatives of the multilinear strong maximal functions, which provide a foundation for our analysis in the continuity part of Theorem 1.1.

Lemma 2.5. Let $f_{i} \in W^{1, p_{i}}\left(\mathbb{R}^{2}\right)$ with $1<p_{i}<\infty$. Then for any $l=1,2$ and almost every $\left(x_{1}, x_{2}\right) \in \mathbb{R}^{2}$, we have

(i) For all $\vec{r} \in \mathcal{B}_{1}(\vec{f})\left(x_{1}, x_{2}\right)$ with $r_{1,1}+r_{1,2}>0, r_{2,1}+r_{2,2}>0$, it holds that

$$
\begin{aligned}
D_{l} \mathscr{M}_{\mathcal{R}} \vec{f}\left(x_{1}, x_{2}\right) & \frac{1}{m} \sum_{\mu=1}^{m} \frac{m}{\left(r_{1,1}+r_{1,2}\right)^{m}\left(r_{2,1}+r_{2,2}\right)^{m}} \prod_{j \neq \mu, 1 \leq j \leq m}^{m} \int_{x_{1}-r_{1,1}}^{x_{1}+r_{1,2}} \int_{x_{2}-r_{2,1}}^{x_{2}+r_{2,2}}\left|f_{j}\left(y_{1}, y_{2}\right)\right| d y_{1} d y_{2} \\
& \times \int_{x_{1}-r_{1,1}}^{x_{1}+r_{1,2}} \int_{x_{2}-r_{2,1}}^{x_{2}+r_{2,2}} D_{l}\left|f_{\mu}\left(y_{1}, y_{2}\right)\right| d y_{1} d y_{2}
\end{aligned}
$$


(ii) For all $\vec{r} \in \mathcal{B}_{1}(\vec{f})\left(x_{1}, x_{2}\right) \cup \mathcal{B}_{2}(\vec{f})\left(x_{1}, x_{2}\right)$ with $r_{1,1}+r_{1,2}>0, r_{2,1}=r_{2,2}=0$, we have

$$
\begin{aligned}
& D_{l} \mathscr{M}_{\mathcal{R}} \vec{f}\left(x_{1}, x_{2}\right) \\
& =\sum_{\mu=1}^{m} \frac{1}{\left(r_{1,1}+r_{1,2}\right)^{m}} \prod_{j \neq \mu, 1 \leq j \leq m}^{m} \int_{x_{1}-r_{1,1}}^{x_{1}+r_{1,2}}\left|f_{j}\left(y_{1}, x_{2}\right)\right| d y_{1} \int_{x_{1}-r_{1,1}}^{x_{1}+r_{1,2}} D_{l}\left|f_{\mu}\left(y_{1}, x_{2}\right)\right| d y_{1}
\end{aligned}
$$

(iii) For all $\vec{r} \in \mathcal{B}_{1}(\vec{f})\left(x_{1}, x_{2}\right) \cup \mathcal{B}_{3}(\vec{f})\left(x_{1}, x_{2}\right)$ with $r_{1,1}=r_{1,2}=0, r_{2,1}+r_{2,2}>0$, it holds

$$
\begin{aligned}
& D_{l} \mathscr{M}_{\mathcal{R}} \vec{f}\left(x_{1}, x_{2}\right) \\
& =\sum_{\mu=1}^{m} \frac{1}{\left(r_{2,1}+r_{2,2}\right)^{m}} \prod_{j \neq \mu, 1 \leq j \leq m}^{m} \int_{x_{2}-r_{2,1}}^{x_{2}+r_{2,2}}\left|f_{j}\left(x_{1}, y_{2}\right)\right| d y_{2} \int_{x_{2}-r_{2,1}}^{x_{2}+r_{2,2}} D_{l}\left|f_{\mu}\left(x_{1}, y_{2}\right)\right| d y_{2}
\end{aligned}
$$

(iv) If $\overrightarrow{0} \in \mathcal{B}_{i}(\vec{f})\left(x_{1}, x_{2}\right)$ for $i=1,2,3$, then,

$$
D_{l} \mathscr{M}_{\mathcal{R}} \vec{f}\left(x_{1}, x_{2}\right)=D_{l}|f|\left(x_{1}, x_{2}\right) .
$$

Proof. We may assume without loss of generality that all $f_{i} \geq 0$, since $\left|f_{i}\right| \in W^{1, p_{i}}\left(\mathbb{R}^{2}\right)$ if $f_{i} \in$ $W^{1, p_{i}}\left(\mathbb{R}^{2}\right)$. Fix $\Lambda>0$ and $l \in\{1,2\}$. Invoking Corollary 2.4, for any $i \in\{1,2,3\}$, we can choose a sequence $\left\{s_{i, k}\right\}_{k=1}^{\infty}, s_{i, k}>0$ and $s_{i, k} \rightarrow 0$ such that $\lim _{k \rightarrow \infty} \pi\left(\mathcal{B}_{i}(\vec{f})(x), \mathcal{B}_{i}(\vec{f})\left(x+s_{i, k} \vec{e}_{l}\right)\right)=0$ for a.e. $x \in R_{\vec{\Lambda}}(\overrightarrow{0})$. Then, Step 1 in the proof of Theorem 1.1 yields that $\mathscr{M}_{\mathcal{R}}(\vec{f}) \in W^{1, p}\left(\mathbb{R}^{2}\right)$ and

$$
\left\|\left(\mathscr{M}_{\mathcal{R}}(\vec{f})\right)_{s_{i, k}}^{l}-D_{l} \mathscr{M}_{\mathcal{R}}(\vec{f})\right\|_{L^{p}\left(\mathbb{R}^{2}\right)} \rightarrow 0 \text { as } k \rightarrow \infty
$$

We also see that

$$
\begin{gathered}
\left\|\left(f_{\mu}\right)_{s_{i, k}}^{l}-D_{l} f_{\mu}\right\|_{L^{p}\left(\mathbb{R}^{2}\right)} \rightarrow 0 \text { as } k \rightarrow \infty, \quad\left\|\left(f_{\mu}\right)_{\tau\left(s_{i, k}\right)}^{l}-f_{\mu}\right\|_{L^{p}\left(\mathbb{R}^{2}\right)} \rightarrow 0 \text { as } k \rightarrow \infty, \\
\left\|\mathcal{M}_{\mathcal{R}}\left(\left(f_{\mu}\right)_{s_{i, k}}^{l}-D_{l} f_{\mu}\right)\right\|_{L^{p}\left(\mathbb{R}^{2}\right)} \rightarrow 0 \text { as } k \rightarrow \infty, \quad\left\|\mathcal{M}_{\mathcal{R}}\left(\left(f_{\mu}\right)_{\tau\left(s_{i, k}\right)}^{l}-f_{\mu}\right)\right\|_{L^{p}\left(\mathbb{R}^{2}\right)} \rightarrow 0 \text { as } k \rightarrow \infty, \\
\left\|\widetilde{\mathcal{M}}_{j}\left(\left(f_{\mu}\right)_{s_{i, k}}^{l}-D_{l} f_{\mu}\right)\right\|_{L^{p}\left(\mathbb{R}^{2}\right)} \rightarrow 0 \text { as } k \rightarrow \infty \quad(j=1,2), \\
\left\|\widetilde{\mathcal{M}}_{j}\left(\left(f_{\mu}\right)_{\tau\left(s_{i, k}\right)}^{l}-f_{\mu}\right)\right\|_{L^{p}\left(\mathbb{R}^{2}\right)} \rightarrow 0 \text { as } k \rightarrow \infty \quad(j=1,2),
\end{gathered}
$$

where $\widetilde{\mathcal{M}}_{j}$ is the one dimensional uncentered Hardy-Littlewood maximal operator with respect to the variable $x_{j}(j=1,2)$. Furthermore, there exists a subsequence $\left\{h_{i, k}\right\}_{k=1}^{\infty}$ of $\left\{s_{i, k}\right\}_{k=1}^{\infty}$ and a measurable set $A_{i, 1} \subset R_{\vec{\Lambda}}(\overrightarrow{0})$ such that $\left|R_{\vec{\Lambda}}(\overrightarrow{0}) \backslash A_{i, 1}\right|=0$ and

(i) $\left(f_{\mu}\right)_{h_{i, k}}^{l}(x) \rightarrow D_{l} f_{\mu}(x),\left(f_{\mu}\right)_{\tau\left(h_{i, k}\right)}^{l}(x) \rightarrow f_{\mu}(x), \mathcal{M}_{\mathcal{R}}\left(\left(f_{\mu}\right)_{h_{i, k}}^{l}-D_{l} f_{\mu}\right) \rightarrow 0, \mathcal{M}_{\mathcal{R}}\left(\left(f_{\mu}\right)_{\tau\left(h_{i, k}\right)}^{l}-\right.$ $\left.f_{\mu}\right) \rightarrow 0, \widetilde{\mathcal{M}}_{j}\left(\left(f_{\mu}\right)_{h_{i, k}}^{l}-D_{l} f_{\mu}\right)(x) \rightarrow 0(j=1,2), \widetilde{\mathcal{M}}_{j}\left(\left(f_{\mu}\right)_{\tau\left(h_{i, k}\right)}^{l}-f_{\mu}\right) \rightarrow 0(j=1,2)$ and $\left(\mathscr{M}_{\mathcal{R}}(\vec{f})\right)_{h_{i, k}}^{l}(x) \rightarrow D_{l} \mathscr{M}_{\mathcal{R}}(\vec{f})(x)$ when $k \rightarrow \infty$ for any $x \in A_{i, 1}$;

(ii) $\left.\lim _{k \rightarrow \infty} \pi\left(\mathcal{B}_{i}(\vec{f})\right)(x), \mathcal{B}_{i}(\vec{f})\left(x+h_{i, k} \vec{e}_{l}\right)\right)=0$ for any $x \in A_{i, 1}$.

Let

$$
\begin{aligned}
A_{i, 2}:= & \bigcap_{k=1}^{\infty}\left\{x \in \mathbb{R}^{2}: \mathscr{M}_{\mathcal{R}}(\vec{f})\left(x+h_{i, k} \vec{e}_{l}\right) \geq u_{x+h_{i, k} \vec{e}_{l}, \vec{f}}(0,0,0,0)\right\}, \\
A_{i, 3}:= & \left\{x \in \mathbb{R}^{2}: \mathscr{M}_{\mathcal{R}}(\vec{f})(x)=u_{x, \vec{f}}(0,0,0,0) \text { if }(0,0,0,0) \in \mathcal{B}_{i}(\vec{f}(x)\},\right. \\
A_{i, 4}:= & \bigcap_{k=1}^{\infty}\left\{x \in \mathbb{R}^{2}: \mathscr{M}_{\mathcal{R}}(\vec{f})\left(x+h_{i, k} \vec{e}_{l}\right)=u_{x+h_{i, k} \vec{e}_{l}, \vec{f}}(0,0,0,0)\right. \\
& \text { if } \left.(0,0,0,0) \in \mathcal{B}_{i}(\vec{f})\left(x+h_{i, k} \vec{e}_{l}\right)\right\},
\end{aligned}
$$




$$
\begin{aligned}
A_{i, 5}:= & \bigcap_{k=1}^{\infty}\left\{x \in \mathbb{R}^{2}: \mathscr{M}_{\mathcal{R}}(\vec{f})\left(x+h_{i, k} \vec{e}_{l}\right)=u_{x+h_{i, k} \vec{e}_{l}, \vec{f}}\left(r_{1,1}, r_{1,2}, 0,0\right)\right. \\
& \text { if } \left.\left(r_{1,1}, r_{1,2}, 0,0\right) \in \mathcal{B}_{1}(\vec{f})\left(x+h_{i, k} \vec{e}_{l}\right) \text { and } r_{1,1}+r_{1,2}>0\right\}, \\
A_{i, 6}:= & \left\{x \in \mathbb{R}^{2}: \mathscr{M}_{\mathcal{R}}(\vec{f})(x)=u_{x, \vec{f}}\left(r_{1,1}, r_{1,2}, 0,0\right) \text { if }\left(r_{1,1}, r_{1,2}, 0,0\right) \in \mathcal{B}_{1}(\vec{f})(x)\right. \\
& \text { and } \left.r_{1,1}+r_{1,2}>0\right\}, \\
A_{i, 7}:= & \bigcap_{k=1}^{\infty}\left\{x \in \mathbb{R}^{2}: \mathscr{M}_{\mathcal{R}}(\vec{f})\left(x+h_{i, k} \vec{e}_{l}\right)=u_{x+h_{i, k} \vec{e}_{l}, \vec{f}}\left(0,0, r_{2,1}, r_{2,2}\right)\right. \\
& \text { if } \left.\left(0,0, r_{2,1}, r_{2,2}\right) \in \mathcal{B}_{1}(\vec{f})\left(x+h_{i, k} \vec{e}_{l}\right) \text { and } r_{2,1}+r_{2,2}>0\right\}, \\
A_{i, 8}:= & \left\{x \in \mathbb{R}^{2}: \mathscr{M}_{\mathcal{R}}(\vec{f})(x)=u_{x, \vec{f}}\left(0,0, r_{2,1}, r_{2,2}\right) \text { if }\left(0,0, r_{2,1}, r_{2,2}\right) \in \mathcal{B}_{1}(\vec{f})(x)\right. \\
& \text { and } \left.r_{2,1}+r_{2,2}>0\right\}, \\
A_{i, 9}:= & \bigcap_{k=1}^{\infty}\left\{x \in \mathbb{R}^{2}: \mathscr{M}_{\mathcal{R}}(\vec{f})\left(x+h_{i, k} \vec{e}_{l}\right)=u_{x+h_{i, k} \vec{e}_{l}, \vec{f}}(\vec{r}) \text { if } \vec{r} \in \mathcal{B}_{2}(\vec{f})\left(x+h_{i, k} \vec{e}_{l}\right)\right\} ; \\
A_{i, 10}:= & \left\{x \in \mathbb{R}^{2}: \mathscr{M}_{\mathcal{R}}(\vec{f})(x)=u_{x, \vec{f}}(\vec{r}) \text { if } \vec{r} \in \mathcal{B}_{2}(\vec{f})(x)\right\} ; \\
A_{i, 11}:= & \bigcap_{k=1}^{\infty}\left\{x \in \mathbb{R}^{2}: \mathscr{M}_{\mathcal{R}}(\vec{f})\left(x+h_{i, k} \vec{e}_{l}\right)=u_{x+h_{i, k} \vec{e}_{l}, \vec{f}}(\vec{r}) \text { if } \vec{r} \in \mathcal{B}_{3}(\vec{f})\left(x+h_{i, k} \vec{e}_{l}\right)\right\} ; \\
A_{i, 12}:= & \left\{x \in \mathbb{R}^{2}: \mathscr{M}_{\mathcal{R}}(\vec{f})(x)=u_{x, \vec{f}}(\vec{r}) \text { if } \vec{r} \in \mathcal{B}_{3}(f)(x)\right\} .
\end{aligned}
$$

Let $A_{i}=\bigcap_{k=1}^{12} A_{i, k}$ and $A=A_{1} \cup A_{2} \cup A_{3}$. Note that $\left|R_{\vec{\Lambda}}(\overrightarrow{0}) \backslash A\right|=0$. Let $x=\left(x_{1}, x_{2}\right) \in A$ be a Lebesgue point of all $f_{\mu}$ and $D_{l} f_{\mu}$ and $\vec{r}=\left(r_{1,1}, r_{1,2}, r_{2,1}, r_{2,2}\right) \in \mathcal{B}_{i}(\vec{f})(x)$. There exists $\vec{r}_{k}^{i}=$ $\left(r_{1,1, i, k}, r_{1,2, i, k}, r_{2,1, i, k}, r_{2,2, i, k}\right) \in \mathcal{B}_{i}(\vec{f})\left(x+h_{i, k} \vec{e}_{l}\right)$ such that $\lim _{k \rightarrow \infty}\left(r_{1,1, i, k}, r_{1,2, i, k}, r_{2,1, i, k}, r_{2,2, i, k}\right)=$ $\left(r_{1,1}, r_{1,2}, r_{2,1}, r_{2,2}\right)$. Furthermore, we assume that $x_{1}$ is a Lebesgue point of $D_{l} f_{\mu}\left(\cdot, x_{2}\right)$ for $i=2$,

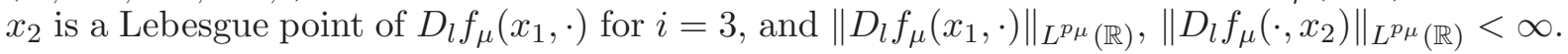

Case $\mathbf{A}\left(r_{1,1}+r_{1,2}>0\right.$ and $\left.r_{2,1}+r_{2,2}>0\right)$. In this case $\vec{r} \in \mathcal{B}_{1}(\vec{f})(x)$ and this happens when $x \in A_{1}$. Without loss of generality we may assume that all $r_{1,1,1, k}>0, r_{1,2,1, k}>0$, $r_{2,1,1, k}>0$ and $r_{2,2,1, k}>0$. Denote $\left[x_{1}-r_{1,1,1, k}, x_{1}+r_{1,2,1, k}\right] \times\left[x_{2}-r_{2,1,1, k}, x_{2}+r_{2,2,1, k}\right]$ by $R_{k}$ and $d y_{1} d y_{2}=d \vec{y}$. Then, noting $\vec{r}_{k} \in \mathcal{B}_{1}(\vec{f})\left(x+h_{1, k} \vec{e}_{l}\right)$ and using Lemma 2.2 (ix), we have

$$
\begin{aligned}
& D_{l} \mathscr{M}_{\mathcal{R}}(\vec{f})(x)=\lim _{k \rightarrow \infty} \frac{1}{h_{1, k}}\left(\mathscr{M}_{\mathcal{R}}(\vec{f})\left(x+h_{1, k} \vec{e}_{l}\right)-\mathscr{M}_{\mathcal{R}}(\vec{f})(x)\right) \\
& \left.\leq \lim _{k \rightarrow \infty} \frac{1}{h_{1, k}}\left(u_{x+h_{1, k} \vec{e}_{l}, \vec{f}} \vec{r}_{k}^{1}\right)-u_{x, \vec{f}}\left(\vec{r}_{k}^{1}\right)\right) \\
& =\lim _{k \rightarrow \infty} \sum_{\mu=1}^{m} \frac{1}{\left(r_{1,1,1, k}+r_{1,2,1, k}\right)^{m}\left(r_{2,1,1, k}+r_{2,2,1, k}\right)^{m}} \iint_{R_{k}}\left(f_{\mu}\right)_{h_{1, k}}^{l}\left(y_{1}, y_{2}\right) d y_{1} d y_{2} \\
& \quad \times \prod_{\nu=1}^{\mu-1} \iint_{R_{k}}\left(f_{\nu}\right)_{\tau\left(h_{1, k}\right)}^{l}\left(y_{1}, y_{2}\right) d y_{1} d y_{2} \prod_{w=\mu+1}^{m} \iint_{R_{k}} f_{w}\left(y_{1}, y_{2}\right) d y_{1} d y_{2} \\
& =\sum_{\mu=1}^{m} \frac{1}{\left(r_{1,1}+r_{1,2}\right)^{m}\left(r_{2,1}+r_{2,2}\right)^{m}} \int_{x_{1}-r_{1,1}}^{x_{1}+r_{1,2}} \int_{x_{2}-r_{2,1}}^{x_{2}+r_{2,2}} D_{l} f_{\mu}\left(y_{1}, y_{2}\right) d \vec{y} \\
& \quad \times \prod_{\nu=1}^{m-1} \int_{x_{1}-r_{1,1}}^{x_{1}+r_{1,2}} \int_{x_{2}-r_{2,1}}^{x_{2}+r_{2,2}} f_{\nu}\left(y_{1}, y_{2}\right) d \vec{y} \prod_{w=\mu+1}^{m} \int_{x_{1}-r_{1,1}}^{x_{1}+r_{1,2}} \int_{x_{2}-r_{2,1}}^{x_{2}+r_{2,2}} f_{w}\left(y_{1}, y_{2}\right) d \vec{y} .
\end{aligned}
$$

Here, we used the fact that $\lim _{k \rightarrow \infty} \vec{r}_{k}^{1}=\vec{r}$ and $\left(f_{\mu}\right)_{\tau\left(h_{1, k}\right)}^{l} \chi_{R_{k}} \rightarrow f_{\mu} \chi_{\left[x_{1}-r_{1,1}, x_{1}+r_{1,2}\right] \times\left[x_{2}-r_{2,1}, x_{2}+r_{2,2}\right]}$ and $\left(f_{\mu}\right)_{h_{1, k}}^{l} \chi_{R_{k}} \rightarrow D_{l} f_{\mu} \chi_{\left[x_{1}-r_{1,1}, x_{1}+r_{1,2}\right] \times\left[x_{2}-r_{2,1}, x_{2}+r_{2,2}\right]}$ in $L^{1}\left(\mathbb{R}^{2}\right)$ as $k \rightarrow \infty$. Then 


$$
\begin{aligned}
D_{l} \mathscr{M}_{\mathcal{R}} \vec{f}(x) \leq & \sum_{\mu=1}^{m} \frac{1}{\left(r_{1,1}+r_{1,2}\right)^{m}\left(r_{2,1}+r_{2,2}\right)^{m}} \int_{x_{1}-r_{1,1}}^{x_{1}+r_{1,2}} \int_{x_{2}-r_{2,1}}^{x_{2}+r_{2,2}} D_{l} f_{\mu}\left(y_{1}, y_{2}\right) d y_{1} d y_{2} \\
& \times \prod_{\nu \neq \mu, 1 \leq \nu \leq m} \int_{x_{1}-r_{1,1}}^{x_{1}+r_{1,2}} \int_{x_{2}-r_{2,1}}^{x_{2}+r_{2,2}} f_{\nu}\left(\left(y_{1}, y_{2}\right)\right) d y_{1} d y_{2} .
\end{aligned}
$$

On the other hand, using Lemma 2.2 (ix), we have

$$
\begin{aligned}
& D_{l} \mathscr{M}_{\mathcal{R}}(\vec{f})(x)=\lim _{k \rightarrow \infty} \frac{1}{h_{1, k}}\left(\mathscr{M}_{\mathcal{R}} \vec{f}\left(x+h_{1, k} \vec{e}_{l}\right)-\mathscr{M}_{\mathcal{R}}(\vec{f})(x)\right) \\
& \geq \lim _{k \rightarrow \infty} \frac{1}{h_{1, k}}\left(u_{x+h_{1, k} \vec{e}_{l}, \vec{f}}(\vec{r})-u_{x, \vec{f}}(\vec{r})\right) \\
& =\sum_{\mu=1}^{m} \frac{1}{\left(r_{1,1}+r_{1,2}\right)^{m}\left(r_{2,1}+r_{2,2}\right)^{m}} \int_{x_{1}-r_{1,1}}^{x_{1}+r_{1,2}} \int_{x_{2}-r_{2,1}}^{x_{2}+r_{2,2}} D_{l} f_{\mu}\left(y_{1}, y_{2}\right) d y_{1} d y_{2} \\
& \quad \times \prod_{1 \leq \nu \neq \mu \leq m} \int_{x_{1}-r_{1,1}}^{x_{1}+r_{1,2}} \int_{x_{2}-r_{2,1}}^{x_{2}+r_{2,2}} f_{\nu}\left(\left(y_{1}, y_{2}\right)\right) d y_{1} d y_{2} .
\end{aligned}
$$

Combining (2.19) with (2.18) yields (2.13) for a.e. $x \in R_{\vec{\Lambda}}(\overrightarrow{0}) \cap A_{1}$.

Case $\mathbf{B}\left(r_{1,1}+r_{1,2}>0\right.$ and $\left.r_{2,1}=r_{2,2}=0\right)$. We consider the following two cases.

(i) $\left(r_{1,1}, r_{1,2}, 0,0\right) \in \mathcal{B}_{2}(\vec{f})(x)$. This happens in the case $x \in A_{2}$. Without loss of generality we may assume that all $r_{1,1,2, k}, r_{1,2,2, k}>0$. We notice that $r_{2,1,2, k}=r_{2,2,2, k}=0$ for all $k \geq 1$. Then, noting $\vec{r}_{k} \in \mathcal{B}_{2}(\vec{f})\left(x+h_{2, k} \vec{e}_{l}\right)$ and using Lemma 2.2 (vii), we have

$$
\begin{aligned}
& D_{l} \mathscr{M}_{\mathcal{R}}(\vec{f})(x)=\lim _{k \rightarrow \infty} \frac{1}{h_{2, k}}\left(\mathscr{M}_{\mathcal{R}}(\vec{f})\left(x+h_{2, k} \vec{e}_{l}\right)-\mathscr{M}_{\mathcal{R}}(\vec{f})(x)\right) \\
& \leq \lim _{k \rightarrow \infty} \frac{1}{h_{2, k}}\left(u_{x+h_{2, k} \overrightarrow{e_{l}, \vec{f}}}\left(r_{1,1,2, k}, r_{1,2,2, k}, 0,0\right)-u_{x, \vec{f}}\left(r_{1,1,2, k}, r_{1,2,2, k}, 0,0\right)\right) \\
& \leq \lim _{k \rightarrow \infty} \frac{1}{h_{2, k}} \sum_{\mu=1}^{m} \frac{1}{\left(r_{1,1,2, k}+r_{1,2,2, k}\right)^{m}} \int_{x_{1}-r_{1,1,2, k}}^{x_{1}+r_{1,2,2, k}}\left(f_{\mu}\right)_{h_{2, k}}^{l}\left(y_{1}, x_{2}\right) d y_{1} \\
& \quad \times \prod_{\nu=1}^{\mu-1} \int_{x_{1}-r_{1,1,2, k}}^{x_{1}+r_{1,2,2, k}}\left(f_{\nu}\right)_{\tau\left(h_{2, k}\right)}^{l}\left(y_{1}, x_{2}\right) d y_{1} \prod_{w=\mu+1}^{m} \int_{x_{1}-r_{1,1,2, k}}^{x_{1}+r_{1,2,2, k}} f_{w}\left(y_{1}, x_{2}\right) d y_{1} \\
& \leq \sum_{\mu=1}^{m} \frac{1}{\left(r_{1,1}+r_{1,2}\right)^{m}} \int_{x_{1}-r_{1,1}}^{x_{1}+r_{1,2}} D_{l} f_{\mu}\left(y_{1}, x_{2}\right) d y_{1} \prod_{\nu \neq \mu, 1 \leq \nu \leq m}^{x_{1}+r_{1,2}} \int_{x_{1}-r_{1,1}} f_{\nu}\left(y_{1}, x_{2}\right) d y_{1} .
\end{aligned}
$$

Here we used the fact that $\lim _{k \rightarrow \infty} r_{1,1,2, k}=r_{1,1}, \lim _{k \rightarrow \infty} r_{1,2,2, k}=r_{1,2}$ and

$$
\left(f_{\mu}\right)_{h_{2, k}}^{l}\left(\cdot, x_{2}\right) \chi_{\left[x_{1}-r_{1,1,2, k}, x_{1}+r_{1,2,2, k}\right]} \rightarrow D_{l} f_{\mu}\left(\cdot, x_{2}\right) \chi_{\left[x_{1}-r_{1,1}, x_{1}+r_{1,2}\right]} \text { in } L^{1}(\mathbb{R}) \text { as } k \rightarrow \infty \text {. }
$$

Further more, using Lemma 2.2 (vii), we have

$$
\begin{aligned}
& D_{l} \mathscr{M}_{\mathcal{R}}(\vec{f})(x) \\
& \geq \lim _{k \rightarrow \infty} \frac{1}{h_{2, k}}\left(u_{x+h_{2, k} e_{l}, \vec{f}}\left(r_{1,1}, r_{1,2}, 0,0\right)-u_{x, \vec{f}}\left(r_{1,1}, r_{1,2}, 0,0\right)\right) \\
& \geq \lim _{k \rightarrow \infty} \sum_{\mu=1}^{m} \frac{1}{\left(r_{1,1,2, k}+r_{1,2,2, k}\right)^{m}} \int_{x_{1}-r_{1,1,2, k}}^{x_{1}+r_{1,2,2, k}}\left(f_{\mu}\right)_{h_{2, k}}^{l}\left(y_{1}, x_{2}\right) d y_{1} \\
& \quad \times \prod_{\nu=1}^{\mu-1} \int_{x_{1}-r_{1,1,2, k}}^{x_{1}+r_{1,2,2, k}}\left(f_{\nu}\right)_{\tau\left(h_{2, k}\right)}^{l}\left(\left(y_{1}, x_{2}\right)\right) d y_{1} \prod_{w=\mu+1}^{m} \int_{x_{1}-r_{1,1,2, k}}^{x_{1}+r_{1,2,2, k}} f_{w}\left(\left(y_{1}, x_{2}\right)\right) d y_{1}
\end{aligned}
$$




$$
\geq \sum_{\mu=1}^{m} \frac{1}{\left(r_{1,1}+r_{1,2}\right)^{m}} \int_{x_{1}-r_{1,1}}^{x_{1}+r_{1,2}} D_{l} f_{\mu}\left(y_{1}, x_{2}\right) d y_{1} \prod_{\nu \neq \mu, 1 \leq \nu \leq m} \int_{x_{1}-r_{1,1}}^{x_{1}+r_{1,2}} f_{\nu}\left(y_{1}, x_{2}\right) d y_{1} .
$$

(2.21) together with (2.20) yields (2.14) for a.e. $x \in R_{\vec{\Lambda}}(\overrightarrow{0}) \cap A_{2}$.

(ii) $\left(r_{1,1}, r_{1,2}, 0,0\right) \in \mathcal{B}_{1}(\vec{f})(x)$. This happens in the case $x \in A_{1}$. Assume that $r_{1,1,1, k}, r_{1,2,1, k}>$ 0 . As in the case $\mathrm{A}$, noting $x \in A_{1} \subset A_{1,5}$, we have

$$
\begin{aligned}
D_{l} \mathscr{M}_{\mathcal{R}}(\vec{f})(x) \leq & \lim _{k \rightarrow \infty} \sum_{\mu=1}^{m} \frac{1}{\left(r_{1,1,1, k}+r_{1,2,1, k}\right)^{m}} \int_{x-r_{1,1,1, k}}^{x+r_{1,2,1, k}}\left(f_{\mu}\right)_{h_{1, k}}^{l}\left(y_{1}, x_{2}\right) d y_{1} \\
& \times \prod_{\nu=1}^{\mu-1} \int_{x-r_{1,1,1, k}}^{x+r_{1,2,1, k}}\left(f_{\nu}\right)_{\tau\left(h_{1, k}\right)}^{l}\left(y_{1}, x_{2}\right) d y_{1} \prod_{w=\mu+1}^{m} \int_{x-r_{1,1,1, k}}^{x+r_{1,2,1, k}} f_{w}\left(y_{1}, x_{2}\right) d y_{1} .
\end{aligned}
$$

We claim that the limits of the right side will tend to

$$
\sum_{\mu=1}^{m} \frac{1}{\left(r_{1,1}+r_{1,2}\right)^{m}} \int_{x_{1}-r_{1,1}}^{x_{1}+r_{1,2}} D_{l} f_{\mu}\left(y_{1}, x_{2}\right) d y_{1} \prod_{\nu \neq \mu, 1 \leq \nu \leq m} \int_{x_{1}-r_{1,1}}^{x_{1}+r_{1,2}} f_{\nu}\left(y_{1}, x_{2}\right) d y_{1} .
$$

To see this, we only consider the limit of the following parts, since the same reasoning applies to the other terms.

$$
\frac{1}{r_{1,1,1, k}+r_{1,2,1, k}} \int_{x-r_{1,1,1, k}}^{x+r_{1,2,1, k}}\left(f_{\mu}\right)_{h_{1, k}}^{l}\left(y_{1}, x_{2}\right) d y_{1} .
$$

Now, we know from the property (i) for $x \in A_{1}$ that

$$
\begin{aligned}
& \left.\lim _{k \rightarrow \infty} \mid \frac{1}{r_{1,1,1, k}+r_{1,2,1, k}} \int_{x-r_{1,1,1, k}}^{x+r_{1,2,1, k}}\left(f_{\mu}\right)_{h_{1, k}}^{l}\left(y_{1}, x_{2}\right)-D_{l} f_{\mu}\left(y_{1}, x_{2}\right)\right) d y_{1} \mid \\
& \leq \lim _{k \rightarrow \infty} \widetilde{\mathcal{M}}_{1}\left(\left(f_{\mu}\right)_{h_{1, k}}^{l}-D_{l} f_{\mu}\right)\left(x_{1}, x_{2}\right) \\
& \left.\quad+\lim _{k \rightarrow \infty} \mid \frac{1}{r_{1,1,1, k}+r_{1,2,1, k}} \int_{x-r_{1,1,1, k}}^{x+r_{1,2,1, k}} D_{l} f_{\mu}\left(y_{1}, x_{2}\right)-D_{l} f_{\mu}\left(y_{1}, x_{2}\right)\right) d y_{1} \mid \\
& =0 .
\end{aligned}
$$

We see moreover that

$$
\begin{aligned}
& \lim _{k \rightarrow \infty}\left|\left(\frac{1}{r_{1,1,1, k}+r_{1,2,1, k}}-\frac{1}{r_{1,1}+r_{1,2}}\right) \int_{x_{1}-r_{1,1,1, k}}^{x_{1}+r_{1,2,1, k}} D_{l} f_{\mu}\left(y_{1}, x_{2}\right) d y_{1}\right| \\
& \leq \lim _{k \rightarrow \infty}\left(r_{1,1,1, k}+r_{1,2,1, k}\right)\left|\frac{1}{r_{1,1,1, k}+r_{1,2,1, k}}-\frac{1}{r_{1,1}+r_{1,2}}\right| \widetilde{\mathcal{M}}_{1}\left(D_{l} f_{\mu}\right)\left(x_{1}, x_{2}\right)=0 .
\end{aligned}
$$

Noting that $\left.\| D_{\ell} f_{\mu}\right)\left(\cdot, x_{2}\right) \|_{\left.L^{p}(\mathbb{R})\right)}<\infty$, we get

$$
\begin{aligned}
\lim _{k \rightarrow \infty}\left|\frac{1}{r_{1,1}+r_{1,2}} \int_{x_{1}-r_{1,1,1, k}}^{x_{1}+r_{1,2,1, k}} D_{l} f_{\mu}\left(y_{1}, x_{2}\right) d y_{1}-\frac{1}{r_{1,1}+r_{1,2}} \int_{x_{1}-r_{1,1}}^{x_{1}+r_{1,2}} D_{l} f_{\mu}\left(y_{1}, x_{2}\right) d y_{1}\right| \\
\leq \lim _{k \rightarrow \infty} \frac{\left(\left|r_{1,1,1, k}-r_{1,1}\right|+\left|r_{1,2,1, k}-r_{1,2}\right|\right)^{1 / p^{\prime}}}{r_{1,1}+r_{1,2}} \\
\left.\quad \times\left.\left(\int_{x_{1}-\max \left\{r_{1,1}, r_{1,1,1, k}\right\}}^{x_{1}-\min \left\{r_{1,1}, r_{1,1,1, k}\right\}}+\int_{x_{1}+\min \left\{r_{1,2}, r_{1,2,1, k}\right\}}^{x_{1}+\max \left\{r_{1,2}, r_{1,2,1, k}\right\}} \mid D_{l} f_{\mu}\left(y_{1}, x_{2}\right)\right)\right|^{p} d y_{1}\right)^{1 / p} \\
\leq C \lim _{k \rightarrow \infty} \frac{\left(\left|r_{1,1,1, k}-r_{1,1}\right|+\left|r_{1,2,1, k}-r_{1,2}\right|\right)^{1 / p^{\prime}}}{r_{1,1}+r_{1,2}}\left\|D_{l} f_{\mu}\left(\cdot, x_{2}\right)\right\|_{\left.L^{p}(\mathbb{R})\right)}=0 .
\end{aligned}
$$

From (2.22) to (2.25), it follows that 


$$
\lim _{k \rightarrow \infty} \frac{1}{r_{1,1,1, k}+r_{1,2,1, k}} \int_{x_{1}-r_{1,1,1, k}}^{x_{1}+r_{1,2,1, k}}\left(f_{\mu}\right)_{h_{1, k}}^{l}\left(y_{1}, x_{2}\right) d y_{1}=\frac{1}{r_{1,1}+r_{1,2}} \int_{x_{1}-r_{1,1}}^{x_{1}+r_{1,2}} D_{l} f_{\mu}\left(y_{1}, x_{2}\right) d y_{1},
$$

and hence we verified the claim.

On the other hand, noting $x \in A_{1} \subset A_{1,6}$, by the same reasoning as in the case $\mathrm{A}$, we get

$$
\begin{aligned}
D_{l} \mathscr{M}_{\mathcal{R}}(\vec{f})(x) \geq & \sum_{\mu=1}^{m} \frac{1}{\left(r_{1,1}+r_{1,2}\right)^{m}} \int_{x_{1}-r_{1,1},}^{x_{1}+r_{1,2}} D_{l} f_{\mu}\left(y_{1}, x_{2}\right) d y_{1} \prod_{\nu=1}^{\mu-1} \int_{x_{1}-r_{1,1}}^{x_{1}+r_{1,2}} f_{\nu}\left(y_{1}, x_{2}\right) d y_{1} \\
& \times \prod_{w=\mu+1}^{m} \int_{x_{1}-r_{1,1}}^{x_{1}+r_{1,2}} f_{w}\left(y_{1}, x_{2}\right) d y_{1}
\end{aligned}
$$

The above claim and (2.27) yield (2.14) for a.e. $x \in R_{\vec{\Lambda}}(\overrightarrow{0}) \cap A_{1}$.

Case $\mathbf{C}\left(r_{1,1}=r_{1,2}=0\right.$ and $\left.r_{2,1}+r_{2,2}>0\right)$. Similar argument as in Case B gives (2.15) for a.e. $x \in R_{\vec{\Lambda}}(\overrightarrow{0}) \cap\left(A_{1} \cup A_{2}\right)$.

Case $\mathbf{D}(\vec{r}=(0,0,0,0))$. We consider the following three cases:

(i) Assume that $(0,0,0,0) \in \mathcal{B}_{2}(\vec{f})(x)$. Then $x \in A_{2}$. The lower bound of $D_{l} \mathscr{M}_{\mathcal{R}}(\vec{f})(x)$ follows from

$$
\begin{aligned}
& D_{l} \mathscr{M}_{\mathcal{R}}(\vec{f})(x) \\
& =\lim _{k \rightarrow \infty} \frac{1}{h_{2, k}}\left(\mathscr{M}_{\mathcal{R}}(\vec{f})\left(x+h_{2, k} \vec{e}_{l}\right)-\mathscr{M}_{\mathcal{R}}(\vec{f})(x)\right) \\
& \geq \lim _{k \rightarrow \infty} \frac{1}{h_{2, k}}\left(\prod_{\mu=1}^{m} f_{\mu}\left(x+h_{2, k} \vec{e}_{l}\right)-\prod_{\mu=1}^{m} f_{\mu}(x)\right) \\
& \geq \sum_{\mu=1}^{m} \lim _{k \rightarrow \infty} \frac{1}{h_{2, k}}\left(f_{\mu}\left(x+h_{2, k} \vec{e}_{l}\right)-f_{\mu}(x)\right)\left(\prod_{\nu=1}^{\mu-1} f_{\nu}(x)\right)\left(\prod_{j=\mu+1}^{m} f_{j}\left(x+h_{2, k} \vec{e}_{l}\right)\right) \\
& \geq \sum_{\mu=1}^{m} D_{l} f_{\mu}(x)\left(\prod_{i \neq \mu, 1 \leq i \leq m} f_{i}(x)\right) .
\end{aligned}
$$

To get the upper bound of $D_{l} \mathscr{M}_{\mathcal{R}}(\vec{f})(x)$, note that $\lim _{k \rightarrow \infty} r_{1,1,2, k}=0, \lim _{k \rightarrow \infty} r_{1,2,2, k}=0$ and $r_{2,1,2, k}=r_{2,2,2, k}=0$ for all $k \geq 1$. If $r_{1,1, k}+r_{1,2, k}=0$ for infinitely many $k$, then by Lemma 2.2 (iv). one obtains that

$$
\begin{aligned}
D_{l} \mathscr{M}_{\mathcal{R}}(\vec{f})(x) & =\lim _{k \rightarrow \infty} \frac{1}{h_{2, k}}\left(\mathscr{M}_{\mathcal{R}}(\vec{f})\left(x+h_{2, k} \vec{e}_{l}\right)-\mathscr{M}_{\mathcal{R}}(\vec{f})(x)\right) \\
& \leq \lim _{k \rightarrow \infty} \frac{1}{h_{2, k}}\left(\prod_{\mu=1}^{m} f_{\mu}\left(x+h_{2, k} \vec{e}_{l}\right)-\prod_{\mu=1}^{m} f_{\mu}(x)\right) \\
& \leq \sum_{\mu=1}^{m} D_{l} f_{\mu}(x)\left(\prod_{\nu \neq \mu, 1 \leq \nu \leq m} f_{\nu}(x)\right) .
\end{aligned}
$$

If there exists $k_{0} \in \mathbb{N}$ such that $r_{1,1,2, k}+r_{1,2,2, k}>0$ when $k \geq k_{0}$. Then (2.20) gives that

$$
D_{l} \mathscr{M}_{\mathcal{R}}(\vec{f})(x) \leq \sum_{\mu=1}^{m} \lim _{k \rightarrow \infty} \frac{1}{r_{1,1,2, k}+r_{1,2,2, k}} \int_{x_{1}-r_{1,1,2, k}}^{x_{1}+r_{1,2,2, k}}\left(f_{\mu}\right)_{h_{2, k}}^{l}\left(y_{1}, x_{2}\right) d y_{1}\left(\prod_{\nu=1}^{\mu-1} \frac{1}{r_{1,1,2, k}+r_{1,2,2, k}}\right.
$$




$$
\left.\times \int_{x_{1}-r_{1,2,2, k}}^{x_{1}+r_{1,2,2, k}} f_{\nu}\left(y_{1}, x_{2}\right) d y_{1}\right)\left(\prod_{j=\nu+1}^{m} \frac{1}{r_{1,1,2, k}+r_{1,2,2, k}} \int_{x_{1}-r_{1,1,2, k}}^{x_{1}+r_{1,2,2, k}}\left(f_{j}\right)_{\tau\left(h_{2, k}\right)}^{l}\left(y_{1}, x_{2}\right) d y_{1}\right) .
$$

Since $x_{1}$ is a Lebesgue point for $D_{l} f_{\mu}\left(\cdot, x_{2}\right)$, we have

$$
\begin{aligned}
& \lim _{k \rightarrow \infty}\left|\frac{1}{r_{1,1,2, k}+r_{1,2,2, k}} \int_{x_{1}-r_{1,1,2, k}}^{x_{1}+r_{1,2,2, k}}\left(f_{\mu}\right)_{h_{2, k}}^{l}\left(y_{1}, x_{2}\right) d y_{1}-D_{l} f_{\mu}\left(x_{1}, x_{2}\right)\right| \\
& \left.\leq \lim _{k \rightarrow \infty} \frac{1}{r_{1,1,2, k}+r_{1,2,2, k}} \int_{x_{1}-r_{1,1,2, k}}^{x_{1}+r_{1,2,2, k}} \mid\left(f_{\mu}\right)_{h_{2, k}}^{l}\left(y_{1}, x_{2}\right)-D_{l} f_{\mu}\left(y_{1}, x_{2}\right)\right) \mid d y_{1} \\
& \leq \lim _{k \rightarrow \infty} \widetilde{\mathcal{M}}_{1}\left(\left(f_{\mu}\right)_{h_{2, k}}^{l}-D_{l} f_{\mu}\right)(x) \\
& \left.\quad+\lim _{k \rightarrow \infty} \frac{1}{r_{1,1,2, k}+r_{1,2,2, k}} \int_{x_{1}-r_{1,1,2, k}}^{x_{1}+r_{1,2,2, k}} \mid D_{l} f_{\mu}\left(y_{1}, x_{2}\right)-D_{l} f_{\mu}\left(y_{1}, x_{2}\right)\right) \mid d y_{1}=0 .
\end{aligned}
$$

Similarly, it holds that $\lim _{k \rightarrow \infty} \frac{1}{r_{1,1,2, k}+r_{1,2,2, k}} \int_{x_{1}-r_{1,1,2, k}}^{x_{1}+r_{1,2, k}}\left(f_{\mu}\right)_{\tau\left(h_{2, k}\right)}^{l}\left(y_{1}, x_{2}\right) d y_{1}=f_{\mu}\left(x_{1}, x_{2}\right)$.

We get from (2.30) and (2.31) that

$$
D_{l} \mathscr{M}_{\mathcal{R}}(\vec{f})(x) \leq \sum_{\mu=1}^{m} D_{l} f_{\mu}\left(x_{1}, x_{2}\right)\left(\prod_{1 \leq \nu \neq \mu \leq m} f_{\nu}\left(x_{1}, x_{2}\right)\right)
$$

(2.32) together with (2.28)-(2.29) yields (2.16) in the case $\overrightarrow{0} \in \mathcal{B}_{2}(\vec{f})(x)$ for a.e. $x \in R_{\vec{\Lambda}}(\overrightarrow{0})$.

(ii) Assume that $(0,0,0,0) \in \mathcal{B}_{3}(\vec{f})(x)$. We can get (2.16) for almost $x \in R_{\vec{\Lambda}}(\overrightarrow{0})$ similarly.

(iii) Assume that $(0,0,0,0) \in \mathcal{B}_{1}(\vec{f})(x)$. In the case $x \in A_{1}$. Note that

$$
\begin{aligned}
D_{l} \mathscr{M}_{\mathcal{R}}(\vec{f})(x) & =\lim _{k \rightarrow \infty} \frac{1}{h_{1, k}}\left(\mathscr{M}_{\mathcal{R}}(\vec{f})\left(x+h_{1, k} \vec{e}_{l}\right)-\mathscr{M}_{\mathcal{R}}(\vec{f})(x)\right) \\
& \geq \sum_{\mu=1}^{m} D_{l} f_{\mu}(x)\left(\prod_{\nu \neq \mu, 1 \leq \nu \leq m} f_{\nu}(x)\right) .
\end{aligned}
$$

Below we estimate the upper bound of $D_{l} \mathscr{M}_{\mathcal{R}}(\vec{f})(x)$. We consider the following four cases:

(a) If $\left(r_{1,1, k}, r_{1,2, k}, r_{2,1, k}, r_{2,2, k}\right)=(0,0,0,0)$ for infinitely many $k$, then

$$
\begin{aligned}
D_{l} \mathscr{M}_{\mathcal{R}}(\vec{f})(x) & =\lim _{k \rightarrow \infty} \frac{1}{h_{1, k}}\left(\mathscr{M}_{\mathcal{R}}(\vec{f})\left(x+h_{1, k} \vec{e}_{l}\right)-\mathscr{M}_{\mathcal{R}}(\vec{f})(x)\right) \\
& \leq \sum_{\mu=1}^{m} \lim _{k \rightarrow \infty} \frac{1}{h_{1, k}}\left(f_{\mu}\left(x+h_{1, k} \vec{e}_{l}\right)-f_{\mu}(x)\right)\left(\prod_{\nu=1}^{\mu-1} f_{\nu}(x)\right)\left(\prod_{j=\mu+1}^{m} f_{j}\left(x+h_{1, k} \vec{e}_{l}\right)\right) \\
& \leq \sum_{\mu=1}^{m} D_{l} f_{\mu}\left(x_{1}, x_{2}\right)\left(\prod_{i \neq \mu, 1 \leq i \leq m} f_{i}\left(x_{1}, x_{2}\right)\right) .
\end{aligned}
$$

This leads to the desired results.

(b) Denote $\left[x_{1}-r_{1,1,1, k}, x_{1}+r_{1,2,1, k}\right] \times\left[x_{2}-r_{2,1,1, k}, x_{2}+r_{2,2,2, k}\right]$ by $R_{k}$. If there exists $k_{0} \in \mathbb{N}$ such that $r_{1,1,1, k}+r_{1,2,1, k}>0$ and $r_{2,1,1, k}+r_{2,2,1, k}>0$ when $k \geq k_{0}$. Then (2.17) gives that

$$
\begin{aligned}
D_{l} & \mathscr{M}_{\mathcal{R}}(\vec{f})(x) \leq \sum_{\mu=1}^{m} \lim _{k \rightarrow \infty} \frac{1}{\left(r_{1,1,1, k}+r_{1,2,1, k}\right)\left(r_{2,1,1, k}+r_{2,2,1, k}\right)} \iint_{R_{k}}\left(f_{\mu}\right)_{h_{1, k}}^{l}\left(y_{1}, y_{2}\right) d y_{1} d y_{2} \\
& \times\left(\prod_{\nu=1}^{\mu-1} \frac{1}{\left(r_{1,1,1, k}+r_{1,2,1, k}\right)\left(r_{2,1,1, k}+r_{2,2,1, k}\right)} \iint_{R_{k}} f_{\nu}\left(y_{1}, y_{2}\right) d y_{1} d y_{2}\right)
\end{aligned}
$$




$$
\times\left(\prod_{j=\mu+1}^{m} \frac{1}{\left(r_{1,1,1, k}+r_{1,2,1, k}\right)\left(r_{2,1,1, k}+r_{2,2,1, k}\right)} \iint_{R_{k}}\left(f_{j}\right)_{\tau\left(h_{1, k}\right)}^{l}\left(y_{1}, y_{2}\right) d y_{1} d y_{2} .\right.
$$

Since $\left(x_{1}, x_{2}\right)$ is a Lebesgue point for $D_{l} f_{\mu}$, then

$$
\begin{aligned}
& \lim _{k \rightarrow \infty}\left|\frac{1}{\left(r_{1,1,1, k}+r_{1,2,1, k}\right)\left(r_{2,1,1, k}+r_{2,2,1, k}\right)} \iint_{R_{k}}\left(f_{\mu}\right)_{h_{1, k}}^{l}\left(y_{1}, y_{2}\right) d y_{1} d y_{2}-D_{l} f_{\mu}\left(x_{1}, x_{2}\right)\right| \\
& \leq \lim _{k \rightarrow \infty} \mathcal{M}_{\mathcal{R}}\left(\left(f_{\mu}\right)_{h_{1, k}}^{l}-D_{l} f_{\mu}\right)\left(x_{1}, x_{2}\right) \\
& \quad+\lim _{k \rightarrow \infty}\left|\frac{1}{\left(r_{1,1,1, k}+r_{1,2,1, k}\right)\left(r_{2,1,1, k}+r_{2,2,1, k}\right)} \iint_{R_{k}} D_{l} f_{\mu}\left(y_{1}, y_{2}\right)-D_{l} f_{\mu}\left(x_{1}, x_{2}\right) d y_{1} d y_{2}\right| \\
& =0 .
\end{aligned}
$$

Similarly, we have

$$
\lim _{k \rightarrow \infty}\left|\frac{1}{\left(r_{1,1,1, k}+r_{1,1,2, k}\right)\left(r_{1,2,1, k}+r_{1,2,2, k}\right)} \iint_{R_{k}}\left(f_{\mu}\right)_{\tau\left(h_{1, k}\right)}^{l}\left(y_{1}, y_{2}\right) d y_{1} d y_{2}-f_{\mu}\left(x_{1}, x_{2}\right)\right|=0 .
$$

(2.34) together with (2.35)-(2.36) yields the desired estimate.

(c) If there exists $k_{0} \in \mathbb{N}$ such that $r_{1,1,1, k}+r_{1,2,1, k}>0$ when $k \geq k_{0}$ and $r_{2,1,1, k}=r_{2,2,1, k}=0$ for infinitely many $k$. Then we may have

$$
D_{l} \mathscr{M}_{\mathcal{R}}(\vec{f})(x) \leq \sum_{\mu=1}^{m} D_{l} f_{\mu}\left(x_{1}, x_{2}\right)\left(\prod_{\nu \neq \mu, 1 \leq \nu \leq m} f_{\nu}\left(x_{1}, x_{2}\right)\right) .
$$

This shows the desired upper bounds.

(d) If there exists $k_{0} \in \mathbb{N}$ such that $r_{2,1,1, k}+r_{2,2,1, k}>0$ when $k \geq k_{0}$ and $r_{1,1,1, k}=r_{1,2,1, k}=0$ for infinitely many $k$, we can get the upper bounds by the arguments similar to those used in the case (c).

(2.33) together with (a)-(d) yields (2.16) for almost every $x \in R_{\vec{\Lambda}}(\overrightarrow{0})$. Since $\Lambda$ is arbitrary. This proves Lemma 2.5 .

2.2. Proof of Theorem 1.1. We divide the proof into three steps:

Step 1: The boundedness part. Let $1<p_{1}, \ldots, p_{m}, p<\infty$ and $1 / p=\sum_{i=1}^{m} 1 / p_{i}$. Let $\vec{f}=$ $\left(f_{1}, \ldots, f_{m}\right)$ with each $f_{i} \in W^{1, p_{i}}\left(\mathbb{R}^{d}\right)$. For a function $u$ and $y \in \mathbb{R}^{d}$ we define $u_{h}(x)=u(x+h)$. According to [18, Section 7.11] we know that $u \in W^{1, p}\left(\mathbb{R}^{d}\right)$ for $1<p<\infty$ if and only if $u \in L^{p}\left(\mathbb{R}^{d}\right)$ and $\lim \sup _{h \rightarrow 0}\left\|u_{h}-u\right\|_{L^{p}\left(\mathbb{R}^{d}\right)} /|h|<\infty$. Therefore, we have

$$
\limsup _{h \rightarrow 0} \frac{\left\|\left(f_{i}\right)_{h}-f_{i}\right\|_{L^{p}\left(\mathbb{R}^{d}\right)}}{|h|}<\infty, \quad \forall 1 \leq i \leq m .
$$

On the other hand, for any fixed $h \in \mathbb{R}^{d}$ and $x \in \mathbb{R}^{d}$, we have

$$
\begin{aligned}
\left|\left(\mathscr{M}_{\mathcal{R}}(\vec{f})\right)_{h}(x)-\mathscr{M}_{\mathcal{R}}(\vec{f})(x)\right| \leq & \sup _{\substack{R \ni x \\
R \in \mathcal{R}}} \frac{1}{|R|^{m}}\left|\prod_{i=1}^{m} \int_{R}\right| f_{i}(y+h)\left|d y-\prod_{i=1}^{m} \int_{R}\right| f_{i}(y)|d y| \\
\leq & \sum_{i=1}^{m} \sup _{\substack{R \ni \mathcal{R} \\
R \in \mathcal{R}}} \frac{1}{|R|^{m}} \int_{R}\left|f_{i}(y+h)-f_{i}(y)\right| d y \\
& \times\left(\prod_{\mu=1}^{i-1} \int_{R}\left|f_{\mu}(y)\right| d y\right)\left(\prod_{\nu=\mu+1}^{m} \int_{R}\left|f_{i}(y+h)\right| d y\right) \\
\leq & \sum_{i=1}^{m} \mathscr{M}_{\mathcal{R}}\left(\vec{f}_{h}^{i}\right)(x),
\end{aligned}
$$


where $\vec{f}_{h}^{\vec{i}}=\left(f_{1}, \ldots, f_{i-1},\left(f_{i}\right)_{h}-f_{i},\left(f_{i+1}\right)_{h}, \ldots,\left(f_{m}\right)_{h}\right)$. (2.38) together with (1.2) yields that

$$
\begin{aligned}
& \left\|\left(\mathscr{M}_{\mathcal{R}}(\vec{f})\right)_{h}-\mathscr{M}_{\mathcal{R}}(\vec{f})\right\|_{L^{p}\left(\mathbb{R}^{d}\right)} \leq \sum_{i=1}^{m}\left\|\mathscr{M}_{\mathcal{R}}\left(\vec{f}_{h}^{\imath}\right)\right\|_{L^{p}\left(\mathbb{R}^{d}\right)} \\
& \lesssim m, d, p_{1}, \ldots, p_{m} \\
& \sum_{i=1}^{m}\left\|\left(f_{i}\right)_{h}-f_{i}\right\|_{L^{p_{i}\left(\mathbb{R}^{d}\right)}} \prod_{\mu \neq i, 1 \leq \mu \leq m}\left\|f_{\mu}\right\|_{L^{p_{\mu}\left(\mathbb{R}^{d}\right)}} .
\end{aligned}
$$

We get from (2.39) and (2.37) that $\limsup _{h \rightarrow 0} \frac{\left\|\left(\mathscr{M}_{\mathcal{R}}(\vec{f})\right)_{h}-\mathscr{M}_{\mathcal{R}}(\vec{f})\right\|_{L^{p}\left(\mathbb{R}^{d}\right)}}{|h|}<\infty$. This together with the fact that $\mathscr{M}_{\mathcal{R}}(\vec{f}) \in L^{p}\left(\mathbb{R}^{d}\right)$ yields that $\mathscr{M}_{\mathcal{R}}(\vec{f}) \in W^{1, p}\left(\mathbb{R}^{d}\right)$.

Step 2: Pointwise estimate for $\mathscr{M}_{\mathcal{R}}(\vec{f})$. Let $s_{k}(k=1,2, \ldots)$ be an enumeration of positive rational numbers. We can write

$$
\mathscr{M}_{\mathcal{R}}(\vec{f})(x)=\sup _{\vec{r} \in\left(\left\{s_{k}\right\}_{k=1}^{\infty}\right)^{2 d}} \frac{1}{\left|R_{\vec{r}}(x)\right|^{m}} \prod_{i=1}^{m} \int_{R_{\vec{r}}(x)}\left|f_{i}(y)\right| d y,
$$

where $\vec{r}=\left(r_{1}^{-}, \ldots, r_{d}^{-} ; r_{1}^{+}, \ldots, r_{d}^{+}\right)$and $R_{\vec{r}}(x)=\left(x_{1}-r_{1}^{-}, x_{1}+r_{1}^{+}\right) \times \cdots \times\left(x_{d}-r_{d}^{-}, x_{d}+r_{d}^{+}\right)$. Fixing $k \geq 1$, we let $E_{k}=\left\{\vec{r}=\left(r_{1}^{-}, \ldots, r_{d}^{-} ; r_{1}^{+}, \ldots, r_{d}^{+}\right) \in \mathbb{R}_{+}^{2 d} ; r_{i}^{-}, r_{i}^{+} \in\left\{s_{1}, \ldots, s_{k}\right\}, i=1,2, \ldots, d\right\}$. For $k \in\{1,2, \ldots\}$, we define the operator $T_{k}$ by

$$
T_{k}(\vec{f})(x)=\max _{\vec{r} \in E_{k}} \frac{1}{\left|R_{\vec{r}}(x)\right|^{m}} \prod_{i=1}^{m} \int_{R_{\vec{r}}(x)}\left|f_{i}(y)\right| d y .
$$

For any $h \in \mathbb{R}^{d}$, we can write

$$
\begin{aligned}
\left|T_{k}(\vec{f})(x+h)-T_{k}(\vec{f})(x)\right| \leq & \sum_{i=1}^{m} \max _{\vec{r} \in E_{k}} \frac{1}{\left|R_{\vec{r}}(x)\right|^{m}} \int_{R_{\vec{r}}(x)}\left|f_{i}(y+h)-f_{i}(y)\right| d y \\
& \times\left(\prod_{\mu=1}^{i-1} \int_{R_{\vec{r}}(x)}\left|f_{\mu}(y)\right| d y\right)\left(\prod_{\nu=\mu+1}^{m} \int_{R_{\vec{r}}(x)}\left|f_{i}(y+h)\right| d y\right) .
\end{aligned}
$$

This yields that

$$
\left|D_{l}\left(T_{k}(\vec{f})\right)(x)\right| \leq \sum_{i=1}^{m} T_{k}\left(\vec{f}_{i}^{l}\right)(x) \leq \sum_{i=1}^{m} \mathscr{M}_{\mathcal{R}}\left(\vec{f}_{i}^{\grave{l}}\right)(x), \quad \text { for a.e. } x \in \mathbb{R}^{d} .
$$

Here $\vec{f}_{i}^{l}=\left(f_{1}, \ldots, f_{i-1}, D_{l} f_{i}, f_{i+1}, \ldots, f_{m}\right)$. For all $k \geq 1$, by (2.40) and (1.2), it holds that

$$
\begin{aligned}
&\left\|T_{k}(\vec{f})\right\|_{1, p} \leq\left\|T_{k}(\vec{f})\right\|_{L^{p}\left(\mathbb{R}^{d}\right)}+\sum_{l=1}^{d}\left\|D_{l} T_{k}(\vec{f})\right\|_{L^{p}\left(\mathbb{R}^{d}\right)} \\
& \leq\left\|\mathscr{M}_{\mathcal{R}}(\vec{f})\right\|_{L^{p}\left(\mathbb{R}^{d}\right)}+\sum_{l=1}^{d} \sum_{i=1}^{m}\left\|\mathscr{M}_{\mathcal{R}}\left(\vec{f}_{i}^{l}\right)\right\|_{L^{p}\left(\mathbb{R}^{d}\right)} \\
& \lesssim m, p_{1}, \ldots, p_{m} \\
& \prod_{i=1}^{m}\left\|f_{i}\right\|_{1, p_{i}} .
\end{aligned}
$$

This yields that $\left\{T_{k}(\vec{f})\right\}_{k}$ is a bounded sequence in $W^{1, p}\left(\mathbb{R}^{d}\right)$ which converges to $\mathscr{M}_{\mathcal{R}}(\vec{f})$ pointwisely. The weak compactness of Sobolev spaces implies that $\left\{D_{l}\left(T_{k}(\vec{f})\right)\right\}_{k}$ converges to $D_{l}\left(\mathscr{M}_{\mathcal{R}}(\vec{f})\right)$ weakly in $L^{p}\left(\mathbb{R}^{d}\right)$. This together with (2.40) implies that

$$
\left|D_{l} \mathscr{M}_{\mathcal{R}}(\vec{f})(x)\right| \leq \sum_{i=1}^{m} \mathscr{M}_{\mathcal{R}}\left(\vec{f}_{i}^{l}\right)(x), \quad \text { for a.e. } x \in \mathbb{R}^{d} .
$$


Combining this with (1.2) yields that

$$
\begin{aligned}
&\left\|\nabla \mathscr{M}_{\mathcal{R}}(\vec{f})\right\|_{L^{p}\left(\mathbb{R}^{d}\right)} \leq \sum_{l=1}^{d}\left\|D_{l} \mathscr{M}_{\mathcal{R}}(\vec{f})\right\|_{L^{p}\left(\mathbb{R}^{d}\right)} \leq \sum_{l=1}^{d} \sum_{i=1}^{m}\left\|\mathscr{M}_{\mathcal{R}}\left(\vec{f}_{i}^{l}\right)\right\|_{L^{p}\left(\mathbb{R}^{d}\right)} \\
& \lesssim m, d, p_{1}, \ldots, p_{m} \\
& \sum_{i=1}^{m} \sum_{l=1}^{d}\left\|D_{l} f_{i}\right\|_{L^{p_{i}\left(\mathbb{R}^{d}\right)}} \prod_{j \neq i, 1 \leq j \leq m}\left\|f_{j}\right\|_{L^{p_{j}\left(\mathbb{R}^{d}\right)}} .
\end{aligned}
$$

Therefore, it holds that

$$
\left\|\mathscr{M}_{\mathcal{R}}(\vec{f})\right\|_{1, p}=\left\|\mathscr{M}_{\mathcal{R}}(\vec{f})\right\|_{L^{p}\left(\mathbb{R}^{d}\right)}+\left\|\nabla \mathscr{M}_{\mathcal{R}}(\vec{f})\right\|_{L^{p}\left(\mathbb{R}^{d}\right)} \leq C_{m, d, p_{1}, \ldots, p_{m}} \prod_{i=1}^{m}\left\|f_{i}\right\|_{1, p_{i}} .
$$

Step 3: The continuity part. For convenience, we only prove the case $d=2$ and the case $d>2$ is analogous and more complex, we leave the details to the interested reader. Let $\vec{f}=\left(f_{1}, \ldots, f_{m}\right)$ with each $f_{i} \in W^{1, p_{i}}\left(\mathbb{R}^{2}\right)$ for $1<p_{i}<\infty$. Let $\vec{f}_{j}=\left(f_{1, j}, \ldots, f_{m, j}\right)$ such that $f_{i, j} \rightarrow f_{i}$ in $W^{1, p_{i}}\left(\mathbb{R}^{2}\right)$ when $j \rightarrow \infty$. Let $1<p<\infty$ and $1 / p=\sum_{i=1}^{m} 1 / p_{i}$. It follows from (2.6) that $\left\|\mathscr{M}_{\mathcal{R}}\left(\vec{f}_{j}\right)-\mathscr{M}_{\mathcal{R}}(\vec{f})\right\|_{L^{p}\left(\mathbb{R}^{2}\right)} \rightarrow 0$ when $j \rightarrow \infty$. Thus, it suffices to show that, for any $l=1,2, \ldots, d$, it holds that

$$
\left\|D_{l} \mathscr{M}_{\mathcal{R}}\left(\vec{f}_{j}\right)-D_{l} \mathscr{M}_{\mathcal{R}}(\vec{f})\right\|_{L^{p}\left(\mathbb{R}^{2}\right)} \rightarrow 0 \quad \text { when } j \rightarrow \infty
$$

Without loss of generality we may assume that all $f_{i, j} \geq 0$ and $f_{i} \geq 0$.

Given $\epsilon>0$ and $l=1,2$, letting $\vec{f}_{l}^{i}=\left(f_{1}, \ldots, f_{i-1}, D_{l} f_{i}, f_{i+1}, \ldots, f_{m}\right)$, there exists $\Lambda>0$ such that $\sum_{i=1}^{m}\left\|\mathscr{M}_{\mathcal{R}}\left(\overrightarrow{f_{l}^{i}}\right)\right\|_{p, B_{1}}<\epsilon$ with $B_{1}=\mathbb{R}^{2} \backslash R_{\vec{\Lambda}}(\overrightarrow{0})$. Here $\vec{\Lambda}=(\Lambda, \Lambda)$. By the absolute continuity, there exists $\eta>0$ such that $\sum_{i=1}^{m}\left\|\mathscr{M}_{\mathcal{R}}\left(\vec{f}_{l}^{i}\right)\right\|_{p, A}<\epsilon$ whenever $A$ is a measurable subset of $R_{\vec{\Lambda}}(\overrightarrow{0})$ such that $|A|<\eta$. As we already observed, for a.e. $x \in \mathbb{R}^{2}$, we notice that:

(i) $u_{x, \vec{f}_{l}^{\vec{i}}}$ is continuous on $\overline{\mathbb{R}}_{+}^{4}$ and $\lim _{\substack{\left(r_{1,1}, r_{1,2}, r_{2,1}, r_{2,2}\right) \in \overline{\mathbb{R}}_{+}^{4} \\ r_{1,1}+r_{1,2}+r_{1,2}+r_{2,2} \rightarrow \infty}} u_{x, \vec{f}_{l}^{2}}\left(r_{1,1}, r_{1,2}, r_{2,1}, r_{2,2}\right)=0$;

(ii) $u_{x, \vec{f}_{l}^{i}}\left(r_{1,1}, r_{1,2}, 0,0\right)$ is continuous on $\overline{\mathbb{R}}_{+}^{2}$ and $\lim _{\substack{\left(r_{1,1}, r_{1,2}\right) \in \overline{\mathbb{R}}_{+}^{2} \\ r_{1,1}+r_{1,2} \rightarrow \infty}} u_{x, \vec{f}_{l}^{i}}\left(r_{1,1}, r_{1,2}, 0,0\right)=0$;

(iii) $u_{x, \vec{f}_{l}^{i}}\left(0,0, r_{2,1}, r_{2,2}\right)$ is continuous on $\overline{\mathbb{R}}_{+}^{2}$ and $\lim _{\substack{\left(r_{2,1}, r_{2,2}\right) \in \overline{\mathbb{R}}_{+}^{2} \\ r_{2,1}+r_{2,2} \rightarrow \infty}} u_{x, \vec{f}_{l}^{i}}\left(0,0, r_{2,1}, r_{2,2}\right)=0$.

Then, it follows that for a.e. $x \in \mathbb{R}^{2}$, the function $\sum_{i=1}^{m} u_{x, \vec{f}_{l}^{\imath}}(\cdot, \cdot, \cdot, \cdot)$ is uniformly continuous on $\overline{\mathbb{R}}_{+}^{4}$; the function $\sum_{i=1}^{m} u_{x, \vec{f}_{l}^{i}}(\cdot, \cdot, 0,0)$ is uniformly continuous on $\overline{\mathbb{R}}_{+}^{2}$; the function $\sum_{i=1}^{m} u_{x, \vec{f}_{l}^{\imath}}(0,0, \cdot, \cdot)$ is uniformly continuous on $\overline{\mathbb{R}}_{+}^{2}$. Hence, we can find $\delta_{x}>0$ such that

(iv) If $\left|\overrightarrow{r_{1}}-\overrightarrow{r_{2}}\right|<\delta_{x}$, then $\left|\sum_{i=1}^{m} u_{x, \vec{f}_{l}^{i}}\left(\overrightarrow{r_{1}}\right)-\sum_{i=1}^{m} u_{x, \overrightarrow{f_{l}^{i}}}\left(\overrightarrow{r_{2}}\right)\right|<\left|R_{\vec{\Lambda}}(\overrightarrow{0})\right|^{-1 / p} \epsilon$;

(v) If $\left|r_{1,1,1}-r_{2,1,1}\right|+\left|r_{1,1,2}-r_{2,1,2}\right|<\delta_{x}$, then

$$
\left|\sum_{i=1}^{m} u_{x, \vec{f}_{l}^{\imath}}\left(r_{1,1,1}, r_{1,1,2}, 0,0\right)-\sum_{i=1}^{m} u_{x, \vec{f}_{l}^{\imath}}\left(r_{2,1,1}, r_{2,1,2}, 0,0\right)\right|<\left|R_{\vec{\Lambda}}(\overrightarrow{0})\right|^{-1 / p} \epsilon ;
$$

(vi) If $\left|r_{1,2,1}-r_{2,2,1}\right|+\left|r_{1,2,2}-r_{2,2,2}\right|<\delta_{x}$, then

$$
\left|\sum_{i=1}^{m} u_{x, \vec{f}_{l}^{\imath}}\left(0,0, r_{1,2,1}, r_{1,2,2}\right)-\sum_{i=1}^{m} u_{x, \vec{f}_{l}^{i}}\left(0,0, r_{2,2,1}, r_{2,2,2}\right)\right|<\left|R_{\vec{\Lambda}}(\overrightarrow{0})\right|^{-1 / p} \epsilon .
$$


Now we can write

$$
R_{\vec{\Lambda}}(\overrightarrow{0})=\left(\bigcup_{i=1}^{\infty}\left\{x \in R_{\vec{\Lambda}}(\overrightarrow{0}): \delta_{x}>\frac{1}{i}\right\}\right) \bigcup \mathcal{N}
$$

where $|\mathcal{N}|=0$. It follows that there exists $\delta>0$ such that

$$
\begin{aligned}
& \mid\left\{x \in R_{\vec{\Lambda}}(\overrightarrow{0}):\left|\sum_{i=1}^{m} u_{x, \vec{f}_{l}^{i}}\left(r_{1,1,1}, r_{1,1,2}, 0,0\right)-\sum_{i=1}^{m} u_{x, \vec{f}_{l}^{i}}\left(r_{2,1,1}, r_{2,1,2}, 0,0\right)\right| \geq\left|R_{\vec{\Lambda}}(\overrightarrow{0})\right|^{-1 / p} \epsilon\right. \\
& \left.\quad \text { for some } r_{1,1,1}, r_{1,1,2}, r_{2,1,1}, r_{2,1,2} \text { with }\left|r_{1,1,1}-r_{2,1,1}\right|+\left|r_{1,1,2}-r_{2,1,2}\right|<\delta\right\} \mid \\
& =:\left|B_{2,2}\right|<\frac{\eta}{2} ;
\end{aligned}
$$

$$
\begin{aligned}
& \mid\left\{x \in R_{\vec{\Lambda}}(\overrightarrow{0}):\left|\sum_{i=1}^{m} u_{x, \vec{f}_{l}^{\imath}}\left(0,0, r_{1,2,1}, r_{1,2,2}\right)-\sum_{i=1}^{m} u_{x, \vec{f}_{l}^{i}}\left(0,0, r_{2,2,1}, r_{2,2,2}\right)\right| \geq\left|R_{\vec{\Lambda}}(\overrightarrow{0})\right|^{-1 / p} \epsilon\right. \\
& \left.\quad \text { for some } r_{1,2,1}, r_{1,2,2}, r_{2,2,1}, r_{2,2,2} \text { with }\left|r_{1,2,1}-r_{2,2,1}\right|+\left|r_{1,2,2}-r_{2,2,2}\right|<\delta\right\} \mid \\
& =:\left|B_{2,3}\right|<\frac{\eta}{2} .
\end{aligned}
$$

Applying Lemma 2.3, there exists $j_{1} \in \mathbb{N}$ such that for $i=1,2,3$

$$
\left|\left\{x \in R_{\vec{\Lambda}}(\overrightarrow{0}) ; \mathcal{B}_{i}\left(\vec{f}_{j}\right)(x) \nsubseteq \mathcal{B}_{i}(\vec{f})(x)_{(\delta)}\right\}\right|=:\left|B^{i, j}\right|<\frac{\eta}{2} \text { when } j \geq j_{1}
$$

Let $\vec{f}_{l}, j=\left(f_{1, j}, \ldots, f_{i-1, j}, D_{l} f_{i, j}, f_{i+1, j}, \ldots, f_{m, j}\right)$. Fix $i=1,2,3$. Invoking Lemma 2.5, for a.e. $x \in \mathbb{R}^{2}, j \geq j_{1}$, and for any $\overrightarrow{r_{1}} \in \mathcal{B}_{i}\left(\overrightarrow{f_{j}}\right)(x)$ and $\overrightarrow{r_{2}} \in \mathcal{B}_{i}(\vec{f})(x)$ with $i=1,2,3$, we have

$$
\begin{aligned}
& \left|D_{l} \mathscr{M}_{\mathcal{R}}\left(\overrightarrow{f_{j}}\right)(x)-D_{l} \mathscr{M}_{\mathcal{R}}(\vec{f})(x)\right| \\
& =\left|\sum_{i=1}^{m} u_{x, \overrightarrow{f_{l}}, j}\left(\overrightarrow{r_{1}}\right)-\sum_{i=1}^{m} u_{x, \overrightarrow{f_{l}^{i}}}\left(\overrightarrow{r_{2}}\right)\right| \\
& \leq \sum_{i=1}^{m}\left|u_{x, \vec{f}_{l}^{i}, j}\left(\overrightarrow{r_{1}}\right)-u_{x, \vec{f}_{l}^{i}}\left(\overrightarrow{r_{1}}\right)\right|+\left|\sum_{i=1}^{m} u_{x, \vec{f}_{l}^{i}}\left(\overrightarrow{r_{1}}\right)-\sum_{i=1}^{m} u_{x, \overrightarrow{f_{l}^{i}}}\left(\overrightarrow{r_{2}}\right)\right| .
\end{aligned}
$$

If $x \notin B_{1} \cup B_{2, i} \cup B^{i, j}$, we choose $\overrightarrow{r_{1}} \in \mathcal{B}_{i}\left(\overrightarrow{f_{j}}\right)(x)$ and $\overrightarrow{r_{2}} \in \mathcal{B}_{i}(\vec{f})(x)$ such that $\left|\overrightarrow{r_{1}}-\overrightarrow{r_{2}}\right|<\delta$ and

$$
\left|\sum_{i=1}^{m} u_{x, \vec{f}_{l}^{i}}\left(\overrightarrow{r_{1}}\right)-\sum_{i=1}^{m} u_{x, \vec{f}_{l}^{i}}\left(\overrightarrow{r_{2}}\right)\right|<\left|R_{\vec{\Lambda}}(\overrightarrow{0})\right|^{-1 / p} \epsilon
$$

On the other hand, for any $\overrightarrow{r_{1}} \in \mathcal{B}_{i}\left(\overrightarrow{f_{j}}\right)(x)$ and $\overrightarrow{r_{2}} \in \mathcal{B}_{i}(\vec{f})(x)$, one may obtain that

$$
\left|\sum_{i=1}^{m} u_{x, \vec{f}_{l}^{i}}\left(\overrightarrow{r_{1}}\right)-\sum_{i=1}^{m} u_{x, \vec{f}_{l}^{i}}\left(\overrightarrow{r_{2}}\right)\right| \leq 2 \sum_{i=1}^{m} \mathscr{M}_{\mathcal{R}}\left(\vec{f}_{l}^{\vec{i}}\right)(x), \quad \text { for a.e. } x \in \mathbb{R}^{2} \text {. }
$$

To get the estimate of $\sum_{i=1}^{m}\left|u_{x, \overrightarrow{f_{l}}}, j\left(\overrightarrow{r_{1}}\right)-u_{x, \vec{f}_{l}^{i}}\left(\overrightarrow{r_{1}}\right)\right|$, we consider the following cases: 
Case 1. For simplicity, we denote $\iint_{R_{0}}=\int_{x_{1}-r_{1,1}}^{x_{1}+r_{1,2}} \int_{x_{2}-r_{2,1}}^{x_{2}+r_{2,2}}$. If $\overrightarrow{r_{1}}=\left(r_{1,1}, r_{1,2}, r_{2,1}, r_{2,2}\right) \in \overline{\mathbb{R}}_{+}^{4}$ with $r_{1,1}+r_{1,2}>0$ and $r_{2,1}+r_{2,2}>0$. Then

$$
\begin{aligned}
& \left|u_{x, \overrightarrow{f_{l}}, j}\left(\overrightarrow{r_{1}}\right)-u_{x, \vec{f}_{l}^{i}}\left(\overrightarrow{r_{1}}\right)\right| \\
& =\prod_{w=1}^{2}\left(r_{w, 1}+r_{w, 2}\right)^{-m} \mid\left(\prod_{\mu=1}^{i-1} \iint_{R_{0}} f_{\mu, j}\left(y_{1}, y_{2}\right) d y_{1} d y_{2}\right)\left(\iint_{R_{0}} D_{l} f_{i, j}\left(y_{1}, y_{2}\right) d y_{1} d y_{2}\right) \\
& \times\left(\prod_{\nu=i+1}^{m} \iint_{R_{0}} f_{\nu, j}\left(y_{1}, y_{2}\right) d y_{1} d y_{2}\right) \\
& -\left(\prod_{\mu=1}^{i-1} \iint_{R_{0}} f_{\mu}\left(y_{1}, y_{2}\right) d y_{1} d y_{2}\right)\left(\iint_{R_{0}} D_{l} f_{i}\left(y_{1}, y_{2}\right) d y_{1} d y_{2}\right)\left(\prod_{\nu=i+1}^{m} \iint_{R_{0}} f_{\nu}\left(y_{1}, y_{2}\right) d y_{1} d y_{2}\right) \\
& \leq \sum_{\mu=1}^{i-1} \prod_{w=1}^{2}\left(r_{w, 1}+r_{w, 2}\right)^{-m}\left(\prod_{\ell=1}^{\mu-1} \iint_{R_{0}} f_{\ell}\left(y_{1}, y_{2}\right) d y_{1} y_{2}\right) \iint_{R_{0}}\left|f_{\mu, j}-f_{\mu}\right|\left(y_{1}, y_{2}\right) d y_{1} d y_{2} \\
& \times\left(\prod_{\kappa=\mu+1}^{i-1} \iint R_{0} f_{\kappa, j}\left(y_{1}, y_{2}\right) d y_{1} d y_{2}\right) \iint_{R_{0}} D_{l} f_{i, j}\left(y_{1}, y_{2}\right) d y_{1} d y_{2} \\
& \times\left(\prod_{\tau=i+1}^{m} \iint R_{0} f_{\tau, j}\left(y_{1}, y_{2}\right) d y_{1} d y_{2}\right)+\sum_{\nu=i+1}^{m} \prod_{w=1}^{2}\left(r_{w, 1}+r_{w, 2}\right)^{-m}\left(\prod_{\ell=1}^{i-1} \iint_{R_{0}} f_{\ell}\left(y_{1}, y_{2}\right) d y_{1} d y_{2}\right) \\
& \times \iint_{R_{0}} D_{l} f_{i}\left(y_{1}, y_{2}\right) d y_{1} d y_{2}\left(\prod_{\kappa=i+1}^{\nu-1} \iint_{R_{0}} f_{\kappa}\left(y_{1}, y_{2}\right) d y_{1} d y_{2}\right) \iint_{R_{0}}\left|f_{\nu, j}-f_{\nu}\right|\left(y_{1}, y_{2}\right) d y_{1} d y_{2} \\
& \times\left(\prod_{\tau=\nu+1}^{m} \iint_{R_{0}} f_{\tau, j}\left(y_{1}, y_{2}\right) d y_{1} d y_{2}\right)+\prod_{w=1}^{2}\left(r_{w, 1}+r_{w, 2}\right)^{-m}\left(\prod_{\kappa=1}^{i-1} \iint R_{0} f_{\kappa}\left(y_{1}, y_{2}\right) d y_{1} d y_{2}\right) \\
& \times \iint_{R_{0}}\left|D_{l} f_{i, j}-D_{l} f_{i}\right|\left(y_{1}, y_{2}\right) d y_{1} d y_{2}\left(\prod_{\tau=i+1}^{m} \iint R_{0} f_{\tau, j}\left(y_{1}, y_{2}\right) d y_{1} d y_{2}\right) \\
& \leq \sum_{\mu=1}^{i-1} \mathscr{M}_{\mathcal{R}}\left(\vec{F}_{\mu, j}^{l}\right)(x)+\sum_{\nu=i+1}^{m} \mathscr{M}_{\mathcal{R}}\left(\vec{G}_{\nu, j}^{l}\right)(x)+\mathscr{M}_{\mathcal{R}}\left(\vec{H}_{i, j}^{l}\right)(x)=: \mathcal{G}_{i, j}^{l}(x),
\end{aligned}
$$

where $\vec{F}_{\mu, j}^{l}=\left(f_{1}, \ldots, f_{\mu-1}, f_{\mu, j}-f_{\mu}, f_{\mu+1, j}, \ldots, f_{i-1, j}, D_{l} f_{i, j}, f_{i+1, j}, \ldots, f_{m, j}\right)$, and $\vec{G}_{\nu, j}^{l}, \vec{H}_{i, j}^{l}$ are defined by $\vec{G}_{\nu, j}^{l}=\left(f_{1}, \ldots, f_{i-1}, D_{l} f_{i}, f_{i+1}, \ldots, f_{\nu-1}, f_{\nu, j}-f_{\nu}, f_{\nu+1, j}, \ldots, f_{m, j}\right)$ and $\vec{H}_{i, j}^{l}=$ $\left(f_{1}, \ldots, f_{i-1}, D_{l} f_{i, j}-D_{l} f_{i}, f_{i+1, j}, \ldots, f_{m, j}\right)$.

Case 2. If $\overrightarrow{r_{1}}=(0,0,0,0)$, then

$$
\begin{aligned}
\left|u_{x, \vec{f}_{l}, j}\left(\overrightarrow{r_{1}}\right)-u_{x, \overrightarrow{f_{l}^{i}}}\left(\overrightarrow{r_{1}}\right)\right| \leq & \sum_{\mu=1}^{i-1}\left(\prod_{\ell=1}^{\mu-1} f_{\ell}(x)\right)\left(f_{\mu, j}-f_{\mu}\right)(x)\left(\prod_{\kappa=\mu+1}^{i-1} f_{\kappa, j}(x)\right) D_{l} f_{i, j}(x)\left(\prod_{\tau=i+1}^{m} f_{\tau, j}(x)\right) \\
& +\sum_{\nu=i+1}^{m}\left(\prod_{\ell=1}^{i-1} f_{\ell}(x)\right) D_{l} f_{i}(x)\left(\prod_{\kappa=i+1}^{\nu-1} f_{\kappa}(x)\right)\left(f_{\nu, j}-f_{\nu}\right)(x)\left(\prod_{\tau=\nu+1}^{m} f_{\tau, j}(x)\right) \\
& +\left(\prod_{\kappa=1}^{i-1} f_{\kappa}(x)\right)\left(D_{l} f_{i, j}-D_{l} f_{i}\right)(x)\left(\prod_{\tau=i+1}^{m} f_{\tau, j}(x)\right) .
\end{aligned}
$$


Case 3. If $\overrightarrow{r_{1}}=\left(0,0, r_{2,1}, r_{2,2}\right) \in \overline{\mathbb{R}}_{+}^{4}$ for $r_{2,1}+r_{2,2}>0$, then

$$
\begin{aligned}
& \left|u_{x, \overrightarrow{f_{l}}, j}\left(\overrightarrow{r_{1}}\right)-u_{x, \vec{f}_{l}^{i}}\left(\overrightarrow{r_{1}}\right)\right| \\
& =\frac{1}{\left(r_{2,1}+r_{2,2}\right)^{m}} \mid\left(\prod_{\mu=1}^{i-1} \int_{x_{2}-r_{2,1}}^{x_{2}+r_{2,2}} f_{\mu, j}\left(x_{1}, y_{2}\right) d y_{2}\right) \\
& \times\left(\int_{x_{2}-r_{2,1}}^{x_{2}+r_{2,2}} D_{l} f_{i, j}\left(x_{1}, y_{2}\right) d y_{2}\right)\left(\prod_{\nu=i+1}^{m} \int_{x_{2}-r_{2,1}}^{x_{2}+r_{2,2}} f_{\nu, j}\left(x_{1}, y_{2}\right) d y_{2}\right) \\
& -\left(\prod_{\mu=1}^{i-1} \int_{x_{2}-r_{2,1}}^{x_{2}+r_{2,2}} f_{\mu}\left(x_{1}, y_{2}\right) d y_{2}\right) \\
& \times\left(\int_{x_{2}-r_{2,1}}^{x_{2}+r_{2,2}} D_{l} f_{i}\left(x_{1}, y_{2}\right) d y_{2}\right)\left(\prod_{\nu=i+1}^{m} \int_{x_{2}-r_{2,1}}^{x_{2}+r_{2,2}} f_{\nu}\left(x_{1}, y_{2}\right) d y_{2}\right) \mid \\
& \left.\leq \sum_{\mu=1}^{i-1} \frac{1}{\left(r_{2,1}+r_{2,2}\right)^{m}}\left(\prod_{\ell=1}^{\mu-1} \int_{x_{2}-r_{2,1}}^{x_{2}+r_{2,2}} f_{\ell}\left(x_{1}, y_{2}\right) d y_{2}\right) \int_{x_{2}-r_{2,1}}^{x_{2}+r_{2,2}} \mid f_{\mu, j}-f_{\mu}\right) \mid\left(x_{1}, y_{2}\right) d y_{2} \\
& \times\left(\prod_{\kappa=\mu+1}^{i-1} \int_{x_{2}-r_{2,1}}^{x_{2}+r_{2,2}} f_{\kappa, j}\left(x_{1}, y_{2}\right) d y_{2}\right) \int_{x_{2}-r_{2,1}}^{x_{2}+r_{2,2}} D_{l} f_{i, j}\left(x_{1}, y_{2}\right) d y_{2} \\
& \times\left(\prod_{\tau=i+1}^{m} \int_{x_{2}-r_{2,1}}^{x_{2}+r_{2,2}} f_{\tau, j}\left(x_{1}, y_{2}\right) d y_{2}\right) \\
& +\sum_{\nu=i+1}^{m} \frac{1}{\left(r_{2,1}+r_{2,2}\right)^{m}}\left(\prod_{\ell=1}^{i-1} \int_{x_{2}-r_{2,1}}^{x_{2}+r_{2,2}} f_{\ell}\left(x_{1}, y_{2}\right) d y_{2}\right) \int_{x_{2}-r_{2,1}}^{x_{2}+r_{2,2}} D_{l} f_{i}\left(x_{1}, y_{2}\right) d y_{2} \\
& \times\left(\prod_{\kappa=i+1}^{\nu-1} \int_{x_{2}-r_{2,1}}^{x_{2}+r_{2,2}} f_{\kappa}\left(x_{1}, y_{2}\right) d y_{2}\right) \int_{x_{2}-r_{2,1}}^{x_{2}+r_{2,2}}\left|f_{\nu, j}-f_{\nu}\right|\left(x_{1}, y_{2}\right) d y_{2} \\
& \times\left(\prod_{\tau=\nu+1}^{m} \int_{x_{2}-r_{2,1}}^{x_{2}+r_{2,2}} f_{\tau, j}\left(x_{1}, y_{2}\right) d y_{2}\right) \\
& +\frac{1}{\left(r_{2,1}+r_{2,2}\right)^{m}}\left(\prod_{\kappa=1}^{i-1} \int_{x_{2}-r_{2,1}}^{x_{2}+r_{2,2}} f_{\kappa}\left(x_{1}, y_{2}\right) d y_{2}\right) \int_{x_{2}-r_{2,1}}^{x_{2}+r_{2,2}}\left|D_{l} f_{i, j}-D_{l} f_{i}\right|\left(x_{1}, y_{2}\right) d y_{2} \\
& \times\left(\prod_{\tau=i+1}^{m} \int_{x_{2}-r_{2,1}}^{x_{2}+r_{2,2}} f_{\tau, j}\left(x_{1}, y_{2}\right) d y_{2}\right) \text {. }
\end{aligned}
$$

Case 4. If $\overrightarrow{r_{1}}=\left(r_{1,1}, r_{1,2}, 0,0\right) \in \overline{\mathbb{R}}_{+}^{4}$ with $r_{1,1}+r_{1,2}>0$. Then, similarly as in Case 3 , we can obtain

$$
\begin{aligned}
& \left|u_{x, \vec{f}_{l}^{i, j}}\left(\overrightarrow{r_{1}}\right)-u_{x, \overrightarrow{f_{l}}}\left(\overrightarrow{r_{1}}\right)\right| \\
& \left.\leq \sum_{\mu=1}^{i-1} \frac{1}{\left(r_{1,1}+r_{1,2}\right)^{m}}\left(\prod_{\ell=1}^{\mu-1} \int_{x_{1}-r_{1,1}}^{x_{1}+r_{1,2}} f_{\ell}\left(y_{1}, x_{2}\right) d y_{1}\right) \int_{x_{1}-r_{1,1}}^{x_{1}+r_{1,2}} \mid f_{\mu, j}-f_{\mu}\right) \mid\left(y_{1}, x_{2}\right) d y_{1} \\
& \quad \times\left(\prod_{\kappa=\mu+1}^{i-1} \int_{x_{1}-r_{1,1}}^{x_{1}+r_{1,2}} f_{\kappa, j}\left(y_{1}, x_{2}\right) d y_{1}\right) \int_{x_{1}-r_{1,1}}^{x_{1}+r_{1,2}} D_{l} f_{i, j}\left(y_{1}, x_{2}\right) d y_{1} \\
& \quad \times\left(\prod_{\tau=i+1}^{m} \int_{x_{1}-r_{1,1}}^{x_{1}+r_{1,2}} f_{\tau, j}\left(y_{1}, x_{2}\right) d y_{1}\right)
\end{aligned}
$$




$$
\begin{aligned}
& +\sum_{\nu=i+1}^{m} \frac{1}{\left(r_{1,1}+r_{1,2}\right)^{m}}\left(\prod_{\ell=1}^{i-1} \int_{x_{1}-r_{1,1}}^{x_{1}+r_{1,2}} f_{\ell}\left(y_{1}, x_{2}\right) d y_{1}\right) \int_{x_{1}-r_{1,1}}^{x_{1}+r_{1,2}} D_{l} f_{i}\left(y_{1}, x_{2}\right) d y_{1} \\
& \times\left(\prod_{\kappa=i+1}^{\nu-1} \int_{x_{1}-r_{1,1}}^{x_{1}+r_{1,2}} f_{\kappa}\left(y_{1}, x_{2}\right) d y_{1}\right) \int_{x_{1}-r_{1,1}}^{x_{1}+r_{1,2}}\left|f_{\nu, j}-f_{\nu}\right|\left(y_{1}, x_{2}\right) d y_{1} \\
& \times\left(\prod_{\tau=\nu+1}^{m} \int_{x_{1}-r_{1,1}}^{x_{1}+r_{1,2}} f_{\tau, j}\left(y_{1}, x_{2}\right) d y_{1}\right) \\
& +\frac{1}{\left(r_{1,1}+r_{1,2}\right)^{m}}\left(\prod_{\kappa=1}^{i-1} \int_{x_{1}-r_{1,1}}^{x_{1}+r_{1,2}} f_{\kappa}\left(y_{1}, x_{2}\right) d y_{1}\right) \int_{x_{1}-r_{1,1}}^{x_{1}+r_{1,2}}\left|D_{l} f_{i, j}-D_{l} f_{i}\right|\left(y_{1}, x_{2}\right) d y_{1} \\
& \times\left(\prod_{\tau=i+1}^{m} \int_{x_{1}-r_{1,1}}^{x_{1}+r_{1,2}} f_{\tau, j}\left(y_{1}, x_{2}\right) d y_{1}\right) .
\end{aligned}
$$

Together with the above cases, we obtain

$$
\sum_{i=1}^{m}\left|u_{x, \vec{f}_{l}, j}\left(\overrightarrow{r_{1}}\right)-u_{x, \vec{f}_{l}^{i}}\left(\overrightarrow{r_{1}}\right)\right| \leq \sum_{i=1}^{m} \mathcal{G}_{l}^{i, j}(x)=: \mathcal{G}_{l}^{j}(x), \quad \text { for any } \overrightarrow{r_{1}} \in[0, \infty)^{4} \text {. }
$$

Note that

$$
\lim _{j \rightarrow \infty}\left\|\mathcal{G}_{l}^{i, j}\right\|_{L^{p}\left(\mathbb{R}^{d}\right)}=0
$$

It follows that there exists $j_{2} \in \mathbb{N}$ such that

$$
\left\|\mathcal{G}_{l}^{j}\right\|_{L^{p}\left(\mathbb{R}^{2}\right)}<\epsilon, \quad \forall j \geq j_{2} .
$$

Observe from (2.43)-(2.46) that $\left|B_{2, i} \cup B^{i, j}\right|<\eta$ for all $j \geq j_{1}$ and $i=1,2,3$. These facts together with (2.47)-(2.51) imply that

$$
\begin{aligned}
& \left\|D_{l} \mathscr{M}_{\mathcal{R}}\left(\overrightarrow{f_{j}}\right)-D_{l} \mathscr{M}_{\mathcal{R}}(\vec{f})\right\|_{L^{p}\left(\mathbb{R}^{2}\right)} \\
& \leq\left\|\mathcal{G}_{l}^{j}\right\|_{L^{p}\left(\mathbb{R}^{2}\right)}+\left\|2 \sum_{i=1}^{m} \mathscr{M}_{\mathcal{R}}\left(\overrightarrow{f_{l}^{\imath}}\right)\right\|_{p, B_{1}}+\left\|2 \sum_{i=1}^{m} \mathscr{M}_{\mathcal{R}}\left(\overrightarrow{f_{l}^{\imath}}\right)\right\|_{p, B_{2,1} \cup B^{1, j}}+\left\|2 \sum_{i=1}^{m} \mathscr{M}_{\mathcal{R}}\left(\vec{f}_{l}^{\vec{\imath}}\right)\right\|_{p, B_{2,2} \cup B^{2, j}} \\
& \quad+\left\|2 \sum_{i=1}^{m} \mathscr{M}_{\mathcal{R}}\left(\overrightarrow{f_{l}^{\imath}}\right)\right\|_{p, B_{2,3} \cup B^{3, j}}+\left\|\left|R_{\vec{\Lambda}}(\overrightarrow{0})\right|^{-1 / p} \epsilon\right\|_{p,\left(B_{1} \cup\left(\cup_{i=1}^{3} B_{2, i} \cup B^{i, j}\right)\right)^{c}} \leq 10 \epsilon
\end{aligned}
$$

for all $j \geq \max \left\{j_{1}, j_{2}\right\}$, which leads to (2.42).

\section{Properties on Besov and Triebel-Lizorkin spaces}

This section will be devoted to give the proofs of Theorems 1.2 and 1.3. In what follows, we let $\Delta_{\zeta} f$ denote the difference of $f$, i.e. $\Delta_{\zeta} f(x)=f(x+\zeta)-f(x)$ for all $x, \zeta \in \mathbb{R}^{d}$. We also let $\mathfrak{R}_{d}=\left\{\zeta \in \mathbb{R}^{d} ; 1 / 2<|\zeta| \leq 1\right\}$.

To prove Theorems 1.2 and 1.3, we need the following characterizations of homogeneous Triebel-Lizorkin spaces $\dot{F}_{s}^{p, q}\left(\mathbb{R}^{d}\right)$ and homogeneous Besov spaces $\dot{B}_{s}^{p, q}\left(\mathbb{R}^{d}\right)$.

Lemma 3.1. ([56]). (i) Let $0<s<1,1<p<\infty, 1<q \leq \infty$ and $1 \leq r<\min (p, q)$. Then

$$
\|f\|_{\dot{F}_{s}^{p, q}\left(\mathbb{R}^{d}\right)} \sim\left\|\left(\sum_{k \in \mathbb{Z}} 2^{k s q}\left(\int_{\mathfrak{R}_{d}}\left|\Delta_{2^{-k} \zeta} f\right|^{r} d \zeta\right)^{q / r}\right)^{1 / q}\right\|_{L^{p}\left(\mathbb{R}^{d}\right)}
$$


(ii) Let $0<s<1,1 \leq p<\infty, 1 \leq q \leq \infty$ and $1 \leq r \leq p$. Then

$$
\|f\|_{\dot{B}_{s}^{p, q}\left(\mathbb{R}^{d}\right)} \sim\left(\sum_{k \in \mathbb{Z}} 2^{k s q}\left\|\left(\int_{\mathfrak{R}_{d}}\left|\Delta_{2^{-k} \zeta} f\right|^{r} d \zeta\right)^{1 / r}\right\|_{L^{p}\left(\mathbb{R}^{d}\right)}^{q}\right)^{1 / q} .
$$

Proof of Theorem 1.2. Note that $\|f\|_{B_{s}^{p, q}\left(\mathbb{R}^{d}\right)} \sim\|f\|_{\dot{B}_{s}^{p, q}\left(\mathbb{R}^{d}\right)}+\|f\|_{L^{p}\left(\mathbb{R}^{d}\right)}$ for $s>0$ and $1<p, q<$ $\infty$. For a measurable function $g: \mathbb{R}^{d} \times \mathbb{Z} \times \mathfrak{R}_{d} \rightarrow \mathbb{R}$, we define

$$
\|g\|_{p, q}:=\left(\sum_{k \in \mathbb{Z}} 2^{k s q}\left(\int_{\mathfrak{R}_{d}} \int_{\mathbb{R}^{d}}|g(x, k, \zeta)|^{p} d x d \zeta\right)^{q / p}\right)^{1 / q} .
$$

Using (3.1) with $r=p$ and Fubini's theorem, we have

$$
\|f\|_{\dot{B}_{s}^{p, q}\left(\mathbb{R}^{d}\right)} \sim\left\|\Delta_{2^{-k} \zeta} f\right\|_{p, q}
$$

Let $0<s<1$ and $1<p_{1}, \ldots, p_{m}, p, q<\infty$ with $1 / p=\sum_{i=1}^{m} 1 / p_{i}$. Let $\vec{f}=\left(f_{1}, \ldots, f_{m}\right)$ with each $f_{j} \in B_{s}^{p_{j}, q}\left(\mathbb{R}^{d}\right)$. Fix $\zeta \in \mathbb{R}^{d}$, it is clear that

$$
\mathscr{M}_{\mathcal{R}}(\vec{f})(x+\zeta)=\sup _{\substack{R \ni x+\zeta \\ R \in \mathcal{R}}} \frac{1}{|R|^{m}} \prod_{i=1}^{m} \int_{R}\left|f_{i}(y)\right| d y=\sup _{\substack{R \ni x \\ R \in \mathcal{R}}} \frac{1}{|R|^{m}} \prod_{i=1}^{m} \int_{R}\left|f_{i}(y+\zeta)\right| d y .
$$

One can easily check that

$$
\left|\Delta_{2^{-k} \zeta}\left(\mathscr{M}_{\mathcal{R}}(\vec{f})\right)(x)\right| \leq \sum_{l=1}^{m} \mathscr{M}_{\mathcal{R}}\left(\vec{f}_{l}^{k, \zeta}\right)(x)
$$

where $\vec{f}_{l}^{k, \zeta}=\left(f_{1}, \ldots, f_{l-1}, \Delta_{2^{k} \zeta} f_{l}, f_{l+1}^{k, \zeta}, \ldots, f_{m}^{k, \zeta}\right)$ and $f_{j}^{k, \zeta}(x)=f_{j}\left(x+2^{-k} \zeta\right)$ for all $l+1 \leq j \leq m$. Then we get from (3.2)-(3.3) and Minkowski's inequality that

$$
\begin{aligned}
& \left\|\mathscr{M}_{\mathcal{R}}(\vec{f})\right\|_{\dot{B}_{s}^{p, q}\left(\mathbb{R}^{d}\right)} \\
& \lesssim\left(\sum_{k \in \mathbb{Z}} 2^{k s q}\left(\int_{\mathfrak{R}_{d}} \int_{\mathbb{R}^{d}}\left|\Delta_{2^{-k} \zeta} \mathscr{M}_{\mathcal{R}}(\vec{f})(x)\right|^{p} d x d \zeta\right)^{q / p}\right)^{1 / q} \\
& \lesssim \sum_{l=1}^{m}\left(\sum_{k \in \mathbb{Z}} 2^{k s q}\|\| \mathscr{M}_{\mathcal{R}}\left(\vec{f}_{l}^{k, \zeta}\right)\left\|_{L^{p}\left(\mathbb{R}^{d}\right)}\right\|_{L^{p}\left(\Re_{d}\right)}^{q}\right)^{1 / q} \\
& \lesssim \sum_{l=1}^{m}\left(\sum_{k \in \mathbb{Z}} 2^{k s q}\left\|\prod_{i=1}^{l-1}\right\| f_{i}\left\|_{L^{p_{i}\left(\mathbb{R}^{d}\right)}}\right\| \Delta_{2^{-k} \zeta} f_{l}\left\|_{L^{p_{l}\left(\mathbb{R}^{d}\right)}} \prod_{j=l+1}^{m}\right\| f_{j}^{k, \zeta}\left\|_{L^{p_{j}}\left(\mathbb{R}^{d}\right)}\right\|_{L^{p}\left(\mathfrak{R}_{d}\right)}^{q}\right)^{1 / q} \\
& \lesssim \sum_{l=1}^{m} \prod_{i \neq l, 1 \leq i \leq m}\left\|f_{i}\right\|_{L^{p_{i}\left(\mathbb{R}^{d}\right)}}\left(\sum_{k \in \mathbb{Z}} 2^{k s q}\|\| \Delta_{2^{-k} \zeta} f_{l}\left\|_{L^{p_{l}\left(\mathbb{R}^{d}\right)}}\right\|_{L^{p_{l}\left(\Re_{d}\right)}}^{q}\right)^{1 / q} \\
& \lesssim \sum_{l=1}^{m} \prod_{i \neq l, 1 \leq i \leq m}\left\|f_{i}\right\|_{L^{p_{i}\left(\mathbb{R}^{d}\right)}}\left\|f_{l}\right\|_{\dot{B}_{s}^{p_{l}, q}\left(\mathbb{R}^{d}\right)} .
\end{aligned}
$$

(3.4) together with (1.2) implies that

$$
\left\|\mathscr{M}_{\mathcal{R}}(\vec{f})\right\|_{B_{s}^{p, q}\left(\mathbb{R}^{d}\right)} \leq C \prod_{i=1}^{m}\left\|f_{i}\right\|_{B_{s}^{p_{i}, q}\left(\mathbb{R}^{d}\right)} .
$$

This completes the proof of the boundedness part. 
We now prove the continuity part. Let $\vec{f}_{j}=\left(f_{1, j}, \ldots, f_{m, j}\right)$ and $f_{i, j} \rightarrow f_{i}$ in $B_{s}^{p_{i}, q}\left(\mathbb{R}^{d}\right)$ as $j \rightarrow \infty$. It is known that $f_{i, j} \rightarrow f_{i}$ in $\dot{B}_{s}^{p_{i}, q}\left(\mathbb{R}^{d}\right)$ and in $L^{p_{i}}\left(\mathbb{R}^{d}\right)$ as $j \rightarrow \infty$. One can check that

$$
\left|\mathscr{M}_{\mathcal{R}}\left(\vec{f}_{j}\right)-\mathscr{M}_{\mathcal{R}}(\vec{f})\right| \leq \sum_{l=1}^{m} \mathscr{M}_{\mathcal{R}}\left(\vec{f}^{l}\right)
$$

Here $\vec{f}^{l}=\left(f_{1}, \ldots, f_{l-1}, f_{l, j}-f_{l}, f_{l+1, j}, \ldots, f_{m, j}\right)$. It follows from (3.5) that $\mathscr{M}_{\mathcal{R}}\left(\vec{f}_{j}\right) \rightarrow \mathscr{M}_{\mathcal{R}}(\vec{f})$ in $L^{p}\left(\mathbb{R}^{d}\right)$ as $j \rightarrow \infty$. Therefore, it suffices to show that $\mathscr{M}_{\mathcal{R}}\left(\vec{f}_{j}\right) \rightarrow \mathscr{M}_{\mathcal{R}}(\vec{f})$ in $\dot{B}_{s}^{p, q}\left(\mathbb{R}^{d}\right)$ as $j \rightarrow \infty$. We will prove this claim by contradiction.

Without loss of generality, we may assume that there exists $c>0$ such that

$$
\left\|\mathscr{M}_{\mathcal{R}}\left(\vec{f}_{j}\right)-\mathscr{M}_{\mathcal{R}}(\vec{f})\right\|_{\dot{B}_{s}^{p, q}\left(\mathbb{R}^{d}\right)}>c, \quad \text { for every } j .
$$

It is obvious that $\left\|\Delta_{2^{-k} \zeta}\left(\mathscr{M}_{\mathcal{R}}\left(\vec{f}_{j}\right)-\mathscr{M}_{\mathcal{R}}(\vec{f})\right)\right\|_{L^{p}\left(\mathbb{R}^{d}\right)} \rightarrow 0$ as $j \rightarrow \infty$ for every $(k, \zeta) \in \mathbb{Z} \times \mathfrak{R}_{d}$. By (3.3) , for every $(x, k, \zeta) \in \mathbb{R}^{d} \times \mathbb{Z} \times \Re_{d}$, we have

$$
\begin{aligned}
& \left|\Delta_{2^{-k} \zeta}\left(\mathscr{M}_{\mathcal{R}}\left(\overrightarrow{f_{j}}\right)-\mathscr{M}_{\mathcal{R}}(\vec{f})\right)(x)\right| \\
& \leq\left|\Delta_{2^{-k} \zeta}\left(\mathscr{M}_{\mathcal{R}}\left(\vec{f}_{j}\right)\right)(x)\right|+\left|\Delta_{2^{-k} \zeta}\left(\mathscr{M}_{\mathcal{R}}(\vec{f})\right)(x)\right| \\
& \leq \sum_{l=1}^{m}\left|\mathscr{M}_{\mathcal{R}}\left(\vec{f}_{l, j}^{k, \zeta}\right)(x)-\mathscr{M}_{\mathcal{R}}\left(\vec{f}_{l}^{k, \zeta}\right)(x)\right|+2 \sum_{l=1}^{m} \mathscr{M}_{\mathcal{R}}\left(\vec{f}_{l}^{k, \zeta}\right)(x) .
\end{aligned}
$$

Here $\vec{f}_{l}^{k, \zeta}$ is given as in (3.3) and $\vec{f}_{l, j}^{k, \zeta}=\left(f_{1, j}, \ldots, f_{l-1, j}, \Delta_{2-k} f_{l, j}, f_{l+1, j}^{k, \zeta}, \ldots, f_{m, j}^{k, \zeta}\right)$ with $f_{i, j}^{k, \zeta}(x)=$ $f_{i, j}\left(x+2^{-k} \zeta\right)$ for all $l+1 \leq i \leq m$. From the third inequality to the last one in (3.4), we obtain

$$
\begin{aligned}
\left\|\sum_{l=1}^{m} \mathscr{M}_{\mathcal{R}}\left(\vec{f}_{l}^{k, \zeta}\right)\right\|_{p, q} & \lesssim\left(\sum_{k \in \mathbb{Z}} 2^{k s q}\left(\int_{\mathfrak{R}_{d}} \int_{\mathbb{R}^{d}}\left|\sum_{l=1}^{m} \mathscr{M}_{\mathcal{R}}\left(\vec{f}_{l}^{k, \zeta}\right)(x)\right|^{p} d x d \zeta\right)^{q / p}\right)^{1 / q} \\
& \lesssim \sum_{l=1}^{m}\left(\sum_{k \in \mathbb{Z}} 2^{k s q}\|\| \mathscr{M}_{\mathcal{R}}\left(\vec{f}_{l}^{k, \zeta}\right)\left\|_{L^{p}\left(\mathbb{R}^{d}\right)}\right\|_{L^{p}\left(\Re_{d}\right)}^{q}\right)^{1 / q} \\
& \lesssim \sum_{l=1}^{m} \prod_{i \neq l, 1 \leq i \leq m}\left\|f_{i}\right\|_{L^{p_{i}\left(\mathbb{R}^{d}\right)}\left\|f_{l}\right\|_{\dot{B}_{s}^{p_{l}, q}\left(\mathbb{R}^{d}\right)}}
\end{aligned}
$$

One can also verify that

$$
\left|\mathscr{M}_{\mathcal{R}}\left(\vec{f}_{l, j}^{k, \zeta}\right)-\mathscr{M}_{\mathcal{R}}\left(\vec{f}_{l}^{k, \zeta}\right)\right| \leq \sum_{\mu=1}^{l-1} \mathscr{M}_{\mathcal{R}}\left(I_{\mu, j}^{\vec{k}, \zeta}\right)+\sum_{\nu=l+1}^{m} \mathscr{M}_{\mathcal{R}}\left(J_{\nu, j}^{\vec{k}, \zeta}\right)+\mathscr{M}_{\mathcal{R}}\left(K_{i, j}^{\vec{k}, \zeta}\right),
$$

where

$$
\begin{gathered}
I_{\mu, j}^{\overrightarrow{k, \zeta}}=\left(f_{1}, \ldots, f_{\mu-1}, f_{\mu, j}-f_{\mu}, f_{\mu+1, j}, \ldots, f_{l-1, j}, \Delta_{2^{-k} \zeta} f_{l, j}, f_{l+1, j}^{k, \zeta}, \ldots, f_{m, j}^{k, \zeta}\right), \\
J_{\nu, j}^{\vec{k}, \zeta}=\left(f_{1}, \ldots, f_{l-1}, \Delta_{2^{k} \zeta} f_{l}, f_{l+1}^{k, \zeta}, \ldots, f_{\nu-1}^{k, \zeta}, f_{\nu, j}^{k, \zeta}-f_{\nu}^{k, \zeta}, f_{\nu+1, j}^{k, \zeta}, \ldots, f_{m, j}^{k, \zeta}\right), \\
K_{i, j}^{\vec{k}, \zeta}=\left(f_{1}, \ldots, f_{l-1}, \Delta_{2^{-k} \zeta}\left(f_{l, j}-f_{l}\right), f_{l+1, j}^{k, \zeta}, \ldots, f_{m, j}^{k, \zeta}\right) .
\end{gathered}
$$

By (3.7) and (3.8), one can deduce that

$$
\left\|\sum_{l=1}^{m}\left|\mathscr{M}_{\mathcal{R}}\left(\vec{f}_{l, j}^{k, \zeta}\right)-\mathscr{M}_{\mathcal{R}}\left(\vec{f}_{l}^{k, \zeta}\right)\right|\right\|_{p, q} \rightarrow 0 \text { as } j \rightarrow \infty .
$$

Thus, we can extract a subsequence such that $\sum_{j=1}^{\infty}\left\|\sum_{l=1}^{m}\left|\mathscr{M}_{\mathcal{R}}\left(\vec{f}_{l, j}^{k, \zeta}\right)-\mathscr{M}_{\mathcal{R}}\left(\vec{f}_{l}^{k, \zeta}\right)\right|\right\|_{p, q}<\infty$. 
Let

$$
H(x, k, \zeta)=\sum_{j=1}^{\infty}\left|\sum_{l=1}^{m} \mathscr{M}_{\mathcal{R}}\left(\vec{f}_{l, j}^{k, \zeta}\right)(x)-\mathscr{M}_{\mathcal{R}}\left(\vec{f}_{l}^{k, \zeta}\right)(x)\right|+2 \sum_{l=1}^{m} \mathscr{M}_{\mathcal{R}}\left(\vec{f}_{l}^{k, \zeta}\right)(x) .
$$

It is easily to check that $\|H\|_{p, q}<\infty$. By (3.6), we get

$$
\left|\Delta_{2^{-k} \zeta}\left(\mathscr{M}_{\mathcal{R}}\left(\vec{f}_{j}\right)-\mathscr{M}_{\mathcal{R}}(\vec{f})\right)(x)\right| \leq H(x, k, \zeta) \text { for a.e. }(x, k, \zeta) \in \mathbb{R}^{d} \times \mathbb{Z} \times \Re_{d} .
$$

Since $\|H\|_{p, q}<\infty$, we have $\int_{\mathbb{R}^{d}} \mid H(x, k, \zeta)^{p} d x<\infty$ for a.e. $(k, \zeta) \in \mathbb{Z} \times \mathfrak{R}_{d}$. By (3.9) and the dominated convergence theorem, for a.e. $(k, \zeta) \in \mathbb{Z} \times \mathfrak{R}_{d}$, it holds that

$$
\lim _{j \rightarrow \infty} \int_{\mathbb{R}^{d}}\left|\Delta_{2^{-k} \zeta}\left(\mathscr{M}_{\mathcal{R}}\left(\vec{f}_{j}\right)-\mathscr{M}_{\mathcal{R}}(\vec{f})\right)(x)\right|^{p} d x=0 .
$$

Using (3.9) and the fact $\|H\|_{p, q}<\infty$ again, we have

$$
\int_{\mathbb{R}^{d}}\left|\Delta_{2^{-k} \zeta}\left(\mathscr{M}_{\mathcal{R}}\left(\vec{f}_{j}\right)-\mathscr{M}_{\mathcal{R}}(\vec{f})\right)(x)\right|^{p} d x \leq \int_{\mathbb{R}^{d}} H(x, k, \zeta)^{p} d x, \quad \text { for a.e }(k, \zeta) \in \mathbb{Z} \times \Re_{d}
$$

and

$$
\int_{\mathfrak{R}_{d}} \int_{\mathbb{R}^{d}} H(x, k, \zeta)^{p} d x d \zeta<\infty \quad \text { for every } k \in \mathbb{Z} .
$$

It follows from (3.10)-(3.12) and the dominated convergence theorem that

$$
\lim _{j \rightarrow \infty}\left(\int_{\Re_{d}} \int_{\mathbb{R}^{d}}\left|\Delta_{2^{-k} \zeta}\left(\mathscr{M}_{\mathcal{R}}\left(\overrightarrow{f_{j}}\right)-\mathscr{M}_{\mathcal{R}}(\vec{f})\right)(x)\right|^{p} d x d \zeta\right)^{1 / p}=0
$$

For every $k \in \mathbb{Z}$, by (3.9) and the fact $\|H\|_{p, q}<\infty$ again, we have

$$
\left(\int_{\mathfrak{R}_{d}} \int_{\mathbb{R}^{d}}\left|\Delta_{2^{-k} \zeta}\left(\mathscr{M}_{\mathcal{R}}\left(\vec{f}_{j}\right)-\mathscr{M}_{\mathcal{R}}(\vec{f})\right)(x)\right|^{p} d x d \zeta\right)^{1 / p} \leq\left(\int_{\mathfrak{R}_{d}} \int_{\mathbb{R}^{d}} H(x, k, \zeta)^{p} d x d \zeta\right)^{1 / p}
$$

and

$$
\left(\sum_{k \in \mathbb{Z}} 2^{k s q}\left(\int_{\mathfrak{R}_{d}} \int_{\mathbb{R}^{d}} H(x, k, \zeta)^{p} d x d \zeta\right)^{q / p}\right)^{1 / q}<\infty .
$$

Using (3.14)-(3.15) and the dominated convergence theorem again, one may obtain

$$
\begin{aligned}
\| \Delta_{2^{-k} \zeta} & \left(\mathscr{M}_{\mathcal{R}}\left(\overrightarrow{f_{j}}\right)-\mathscr{M}_{\mathcal{R}}(\vec{f})\right) \|_{p, q} \\
= & \left(\sum_{k \in \mathbb{Z}} 2^{k s q}\left(\int_{\mathfrak{R}_{d}} \int_{\mathbb{R}^{d}}\left|\Delta_{2^{-k} \zeta}\left(\mathscr{M}_{\mathcal{R}}\left(\overrightarrow{f_{j}}\right)-\mathscr{M}_{\mathcal{R}}(\vec{f})\right)(x)\right|^{p} d x d \zeta\right)^{q / p}\right)^{1 / q} \rightarrow 0 \text { as } j \rightarrow \infty .
\end{aligned}
$$

By (3.2), this yields that $\left\|\mathscr{M}_{\mathcal{R}}\left(\overrightarrow{f_{j}}\right)-\mathscr{M}_{\mathcal{R}}(\vec{f})\right\|_{\dot{B}_{s}^{p, q}\left(\mathbb{R}^{d}\right)} \rightarrow 0$ as $j \rightarrow \infty$, which gives a contradiction. The proof of Theorem 1.2 is finished.

Proof of Theorem 1.3. Given an operator $T$ acting on functions in $\mathbb{R}$, we denote by $T^{j}$, $j=1,2, \ldots, d$, the operator defined on functions in $\mathbb{R}^{d}$ by letting $T$ act on the $j$-th variable while keeping the remaining variables fixed, namely

$$
T^{j} f(x)=T\left(f\left(x_{1}, x_{2}, \ldots, x_{j-1}, \cdot, x_{j+1}, \ldots, x_{d}\right)\right)\left(x_{j}\right) \text { for } x \in \mathbb{R}^{d} .
$$

We also define the operator $\mathcal{T}$ by $\mathcal{T} f(x)=T^{1} \circ T^{2} \circ \ldots \circ T^{d} f(x)$. We need the following lemma.

Lemma 3.2. If $T$ is bounded on $L^{p}\left(\mathbb{R}, \ell^{q}\left(L^{r}\left(\Re_{d}\right)\right)\right)$ for some $1<p, q, r<\infty$, then the operator $\mathcal{T}$ is bounded on $L^{p}\left(\mathbb{R}^{d}, \ell^{q}\left(L^{r}\left(\Re_{d}\right)\right)\right)$. 
Proof. For all $j=1, \ldots, d$, we shall prove the following inequality

$$
\left\|\left(\sum_{i \in \mathbb{Z}}\left\|T^{j} f_{i, \zeta}\right\|_{L^{r}\left(\mathfrak{R}_{d}\right)}^{q}\right)^{1 / q}\right\|_{L^{p}\left(\mathbb{R}^{d}\right)} \leq\|T\|\left\|\left(\sum_{i \in \mathbb{Z}}\left\|f_{i, \zeta}\right\|_{L^{r}\left(\mathfrak{R}_{d}\right)}^{q}\right)^{1 / q}\right\|_{L^{p}\left(\mathbb{R}^{d}\right)} .
$$

Here $\|T\|$ represents the operator norm of $T$ on $L^{p}\left(\mathbb{R}, \ell^{q}\left(L^{r}\left(\mathfrak{R}_{d}\right)\right)\right)$. We only prove (3.16) for $j=1$ and the other cases are analogous. We may write

$$
\begin{aligned}
& \left\|\left(\sum_{i \in \mathbb{Z}}\left\|T^{1} f_{i, \zeta}\right\|_{L^{r}\left(\Re_{d}\right)}^{q}\right)^{1 / q}\right\|_{L^{p}\left(\mathbb{R}^{d}\right)}^{p} \\
& =\int_{\mathbb{R}^{d}}\left(\sum_{i \in \mathbb{Z}}\left\|T^{1} f_{i, \zeta}\right\|_{L^{r}\left(\Re_{d}\right)}^{q}\right)^{p / q} d x \\
& =\int_{\mathbb{R}^{d-1}}\left(\int_{\mathbb{R}}\left(\sum_{i \in \mathbb{Z}}\left(\int_{\mathfrak{R}_{d}}\left|T\left(f_{i, \zeta}\left(\cdot, x_{2}, \ldots, x_{d}\right)\right)\left(x_{1}\right)\right|^{r} d \zeta\right)^{q / r}\right)^{p / q} d x_{1}\right) d x_{2} \ldots d x_{d} \\
& \leq\|T\|^{p} \int_{\mathbb{R}^{d-1}}\left(\int_{\mathbb{R}}\left(\sum_{i \in \mathbb{Z}}\left\|f_{i, \zeta}\left(x_{1}, x_{2}, \ldots, x_{d}\right)\right\|_{L^{r}\left(\Re_{d}\right)}^{q}\right)^{p / q} d x_{1}\right) d x_{2} \ldots d x_{d} \\
& =\|T\|^{p}\left\|\left(\sum_{i \in \mathbb{Z}}\left\|f_{i, \zeta}\right\|_{L^{r}\left(\Re_{d}\right)}^{q}\right)^{1 / q}\right\|_{L^{p}\left(\mathbb{R}^{d}\right)}^{p},
\end{aligned}
$$

which leads to (3.16) for $j=1$. (3.16) together with the definition of $\mathcal{T}$ yields that

$$
\left\|\left(\sum_{i \in \mathbb{Z}}\left\|\mathcal{T} f_{i, \zeta}\right\|_{L^{r}\left(\Re_{d}\right)}^{q}\right)^{1 / q}\right\|_{L^{p}\left(\mathbb{R}^{d}\right)} \leq\|T\|^{d}\left\|\left(\sum_{i \in \mathbb{Z}}\left\|f_{i, \zeta}\right\|_{L^{r}\left(\Re_{d}\right)}^{q}\right)^{1 / q}\right\|_{L^{p}\left(\mathbb{R}^{d}\right)} .
$$

This proves Lemma 3.2 .

The following vector-valued inequalities of the one dimensional uncentered Hardy-Littlewood maximal function will be very useful in the proof of Theorem 1.3 .

Lemma 3.3. ([56]). For any $1<p, q, r<\infty$, it holds that

$$
\left\|\left(\sum_{j \in \mathbb{Z}}\left\|\mathcal{M} f_{j, \zeta}\right\|_{L^{r}\left(\Re_{d}\right)}^{q}\right)^{1 / q}\right\|_{L^{p}(\mathbb{R})} \lesssim_{p, q, r}\left\|\left(\sum_{j \in \mathbb{Z}}\left\|f_{j, \zeta}\right\|_{L^{r}\left(\Re_{d}\right)}^{q}\right)^{1 / q}\right\|_{L^{p}(\mathbb{R})} .
$$

Applying Lemmas 3.2 and 3.3, we can get the following

Lemma 3.4. For any $1<p, q, r<\infty$, it holds that

$$
\left\|\left(\sum_{j \in \mathbb{Z}}\left\|\mathcal{M}_{\mathcal{R}} f_{j, \zeta}\right\|_{L^{r}\left(\Re_{d}\right)}^{q}\right)^{1 / q}\right\|_{L^{p}\left(\mathbb{R}^{d}\right)} \lesssim_{p, q, r}\left\|\left(\sum_{j \in \mathbb{Z}}\left\|f_{j, \zeta}\right\|_{L^{r}\left(\Re_{d}\right)}^{q}\right)^{1 / q}\right\|_{L^{p}\left(\mathbb{R}^{d}\right)} .
$$

Proof. For $j=1, \ldots, d$, we define the operator $M^{j}$ by

$$
M^{j} f\left(x_{1}, x_{2}, \ldots, x_{d}\right)=\sup _{a<x_{j}<b} \frac{1}{b-a} \int_{a}^{b}\left|f\left(x_{1}, \ldots, x_{j-1}, y, x_{j+1}, \ldots, x_{d}\right)\right| d y .
$$

One can easily check that

$$
\begin{gathered}
M^{j} f(x)=\mathcal{M}\left(f\left(x_{1}, x_{2}, \ldots, x_{j-1}, \cdot, x_{j+1}, \ldots, x_{d}\right)\left(x_{j}\right),\right. \\
\mathcal{M}_{\mathcal{R}} f(x) \leq M^{1} \circ M^{2} \circ \cdots \circ M^{d} f(x) .
\end{gathered}
$$

Using (3.17)-(3.18) and Lemmas 3.2 3.3, for all $1<p, q, r<\infty$, we can get

$$
\left\|\left(\sum_{j \in \mathbb{Z}}\left\|\mathcal{M}_{\mathcal{R}} f_{j, \zeta}\right\|_{L^{r}\left(\Re_{d}\right)}^{q}\right)^{1 / q}\right\|_{L^{p}\left(\mathbb{R}^{d}\right)} \lesssim p, q, r\left\|\left(\sum_{j \in \mathbb{Z}}\left\|f_{j, \zeta}\right\|_{L^{r}\left(\Re_{d}\right)}^{q}\right)^{1 / q}\right\|_{L^{p}\left(\mathbb{R}^{d}\right)}
$$


Then Lemma 3.4 is proved.

Proof of Theorem 1.3. Let $0<s<1$ and $1<p_{1}, \ldots, p_{m}, p, q<\infty$ with $1 / p=\sum_{i=1}^{m} 1 / p_{i}$. Let $\vec{f}=\left(f_{1}, \ldots, f_{m}\right)$ with each $f_{j} \in F_{s}^{p_{j}, q}\left(\mathbb{R}^{d}\right)$. One can easily check that (3.3) also holds. We get from (3.3) that

$$
\begin{aligned}
& \left|\Delta_{2^{-k} \zeta}\left(\mathscr{M}_{\mathcal{R}}(\vec{f})\right)(x)\right| \\
& \leq \sum_{l=1}^{m} \mathcal{M}_{\mathcal{R}}\left(\Delta_{2^{-k} \zeta} f_{l}\right)(x) \prod_{\mu=1}^{l-1} \mathcal{M}_{\mathcal{R}} f_{\mu}(x) \prod_{\nu=l+1}^{m} \mathcal{M}_{\mathcal{R}}\left(f_{\nu}^{k, \zeta}\right)(x) \\
& =\sum_{l=1}^{m} \mathcal{M}_{\mathcal{R}}\left(\Delta_{2^{-k} \zeta} f_{l}\right)(x) \prod_{\mu=1}^{l-1} \mathcal{M}_{\mathcal{R}} f_{\mu}(x) \prod_{\nu=l+1}^{m} \mathcal{M}_{\mathcal{R}}\left(\Delta_{2^{-k} \zeta} f_{\nu}+f_{\nu}\right)(x) \\
& \leq \sum_{\emptyset \neq \tau \subset \tau_{m}} \prod_{\mu \in \tau} \mathcal{M}_{\mathcal{R}}\left(\Delta_{2^{-k} \zeta} f_{\mu}\right)(x) \prod_{\nu \in \tau^{\prime}} \mathcal{M}_{\mathcal{R}} f_{\nu}(x),
\end{aligned}
$$

where $\tau_{m}=\{1,2, \ldots, m\}$ and $\tau^{\prime}=\tau_{m} \backslash \tau$ for $\tau \subset \tau_{m}$.

Thus, Lemma 3.1 (i), (3.19) and the Minkowski inequality yield that

$$
\begin{aligned}
& \left\|\mathscr{M}_{\mathcal{R}}(\vec{f})\right\|_{\dot{F}_{s}^{p, q}\left(\mathbb{R}^{d}\right)} \\
& \lesssim\left\|\left(\sum_{k \in \mathbb{Z}} 2^{k s q}\left(\int_{\mathfrak{R}_{d}}\left|\Delta_{2^{-k} \zeta}\left(\mathscr{M}_{\mathcal{R}}(\vec{f})\right)\right| d \zeta\right)^{q}\right)^{1 / q}\right\|_{L^{p}\left(\mathbb{R}^{d}\right)} \\
& \lesssim \sum_{\emptyset \neq \tau \subset \tau_{m}}\left\|\left(\sum_{k \in \mathbb{Z}} 2^{k s q}\left(\int_{\mathfrak{R}_{d}} \prod_{\mu \in \tau} \mathcal{M}_{\mathcal{R}}\left(\Delta_{2^{-k} \zeta} f_{\mu}\right) \prod_{\nu \in \tau^{\prime}} \mathcal{M}_{\mathcal{R}} f_{\nu} d \zeta\right)^{q}\right)^{1 / q}\right\|_{L^{p}\left(\mathbb{R}^{d}\right)} .
\end{aligned}
$$

We shall prove the following estimate.

$$
\begin{aligned}
& \left\|\left(\sum_{k \in \mathbb{Z}} 2^{k s q}\left(\int_{\mathfrak{R}_{d}} \prod_{\mu \in \tau} \mathcal{M}_{\mathcal{R}}\left(\Delta_{2^{-k} \zeta} f_{\mu}\right) \prod_{\nu \in \tau^{\prime}} \mathcal{M}_{\mathcal{R}} f_{\nu} d \zeta\right)^{q}\right)^{1 / q}\right\|_{L^{p}\left(\mathbb{R}^{d}\right)} \\
& =\left\|\left(\sum_{k \in \mathbb{Z}} 2^{k s q}\left(\int_{\mathfrak{R}_{d}} \prod_{\mu \in \tau} \mathcal{M}_{\mathcal{R}}\left(\Delta_{2^{-k} \zeta} f_{\mu}\right) d \zeta\right)^{q}\right)^{1 / q} \prod_{\nu \in \tau^{\prime}} \mathcal{M}_{\mathcal{R}} f_{\nu}\right\|_{L^{p}\left(\mathbb{R}^{d}\right)} \\
& \lesssim \prod_{\mu \in \tau}\left\|f_{\mu}\right\|_{F_{s}^{p_{\mu}, q}\left(\mathbb{R}^{d}\right)} \prod_{\nu \in \tau^{\prime}}\left\|f_{\nu}\right\|_{F_{s}^{p_{\nu}, q}\left(\mathbb{R}^{d}\right)}
\end{aligned}
$$

Let $1 / p_{\tau}=\sum_{\mu \in \tau} 1 / p_{\mu}$. Then, using Hölder's inequality and the $L^{p}$ bounds for $\mathcal{M}_{\mathcal{R}}$ we have

$$
\begin{aligned}
& \left\|\left(\sum_{k \in \mathbb{Z}} 2^{k s q}\left(\int_{\mathfrak{R}_{d}} \prod_{\mu \in \mathcal{\tau}} \mathcal{M}_{\mathcal{R}}\left(\Delta_{2^{-k} \zeta} f_{\mu}\right) d \zeta\right)^{q}\right)^{1 / q} \prod_{\nu \in \tau^{\prime}} \mathcal{M}_{\mathcal{R}} f_{\nu}\right\|_{L^{p}\left(\mathbb{R}^{d}\right)} \\
& \leq\left\|\left(\sum_{k \in \mathbb{Z}} 2^{k s q}\left(\int_{\mathfrak{R}_{d}} \prod_{\mu \in \tau} \mathcal{M}_{\mathcal{R}}\left(\Delta_{2^{-k} \zeta} f_{\mu}\right) d \zeta\right)^{q}\right)^{1 / q}\right\|\left\|_{L^{p_{\tau}\left(\mathbb{R}^{d}\right)}}\right\| \prod_{\nu \in \tau^{\prime}} \mathcal{M}_{\mathcal{R}} f_{\nu} \|_{L^{p_{\tau^{\prime}}\left(\mathbb{R}^{d}\right)}} \\
& \leq\left\|\left(\sum_{k \in \mathbb{Z}} 2^{k s q} \prod_{\mu \in \tau}\left\|\mathcal{M}_{\mathcal{R}}\left(\Delta_{2^{-k} \zeta} f_{\mu}\right)\right\|_{L^{p_{\mu} / p_{\tau}\left(\mathfrak{R}_{d}\right)}}^{q}\right)^{1 / q}\right\|_{L^{p_{\tau}\left(\mathbb{R}^{d}\right)}} \prod_{\nu \in \tau^{\prime}}\left\|\mathcal{M}_{\mathcal{R}} f_{\nu}\right\|_{L^{p_{\nu}\left(\mathbb{R}^{d}\right)}} \\
& \leq\left\|\prod_{\mu \in \tau}\left(\sum_{k \in \mathbb{Z}} 2^{k s q}\left\|\mathcal{M}_{\mathcal{R}}\left(\Delta_{2^{-k} \zeta} f_{\mu}\right)\right\|_{L^{p_{\mu} / p_{\tau}\left(\Re_{d}\right)}}^{p_{\mu} q / p_{\tau}}\right)^{p_{\tau} / p_{\mu} q}\right\|_{L^{p_{\tau}\left(\mathbb{R}^{d}\right)}} \prod_{\nu \in \tau^{\prime}}\left\|f_{\nu}\right\|_{L^{p_{\nu}\left(\mathbb{R}^{d}\right)}} \\
& \leq \prod_{i \in \tau}\left\|\left(\sum_{k \in \mathbb{Z}} 2^{k s q}\left\|\mathcal{M}_{\mathcal{R}}\left(\Delta_{2^{-k} \zeta} f_{\mu}\right)\right\|_{L^{p_{\mu} / p_{\tau}\left(\Re_{d}\right)}}^{p_{\mu} q / p_{\tau}}\right)^{p_{\tau} / p_{\mu} q}\right\|_{L^{p_{\mu}\left(\mathbb{R}^{d}\right)}} \prod_{\nu \in \tau^{\prime}}\left\|f_{\nu}\right\|_{L^{p_{\nu}\left(\mathbb{R}^{d}\right)}}
\end{aligned}
$$




$$
\begin{aligned}
& =\prod_{\mu \in \tau}\left\|\left(\sum_{k \in \mathbb{Z}} 2^{k\left(p_{\tau} s / p_{\mu}\right)\left(p_{\mu} q / p_{\tau}\right)}\left\|\mathcal{M}_{\mathcal{R}}\left(\Delta_{2^{-k} \zeta} f_{\mu}\right)\right\|_{L^{p_{\mu} / p_{\tau}\left(\Re_{d}\right)}}^{p_{\mu} q p_{\tau}}\right)^{p_{\tau} / p_{\mu} q}\right\|_{L^{p_{\mu}\left(\mathbb{R}^{d}\right)}} \prod_{\nu \in \tau^{\prime}}\left\|f_{\nu}\right\|_{L^{p_{\nu}}\left(\mathbb{R}^{d}\right)} \\
& \lesssim \prod_{\mu \in \tau}\left\|\left(\sum_{k \in \mathbb{Z}} 2^{k\left(p_{\tau} s / p_{\mu}\right)\left(p_{\mu} q / p_{\tau}\right)}\left\|\Delta_{2^{-k} \zeta} f_{\mu}\right\|_{L^{p_{\mu}} / p_{\tau}\left(\mathfrak{R}_{d}\right)}^{p_{\mu} q p_{\tau}}\right)^{p_{\tau} / p_{\mu} q}\right\|_{L^{p_{\mu}\left(\mathbb{R}^{d}\right)}} \prod_{\nu \in \tau^{\prime}}\left\|f_{\nu}\right\|_{L^{p_{\nu}}\left(\mathbb{R}^{d}\right)} \\
& \lesssim \prod_{\mu \in \tau}\left\|f_{\mu}\right\|_{\dot{F}_{p_{\tau} s / p_{\mu}}^{p_{\mu}, p_{\mu} q / p_{\tau}}\left(\mathbb{R}^{d}\right)} \prod_{\nu \in \tau^{\prime}}\left\|f_{i}\right\|_{L^{p_{\nu}\left(\mathbb{R}^{d}\right)}} \\
& \leq \prod_{\mu \in \tau}\left\|f_{\mu}\right\|_{F_{p_{\tau} s / p_{\mu}}^{p_{\mu}, p_{\mu} q / p_{\tau}}\left(\mathbb{R}^{d}\right)} \prod_{\nu \in \tau^{\prime}}\left\|f_{\nu}\right\|_{L^{p_{\nu}}\left(\mathbb{R}^{d}\right)} \\
& \leq \prod_{\mu \in \tau}\left\|f_{\mu}\right\|_{F_{s}^{p_{\mu}, q}\left(\mathbb{R}^{d}\right)} \prod_{\nu \in \tau^{\prime}}\left\|f_{\nu}\right\|_{F_{s}^{p_{\nu}, q}\left(\mathbb{R}^{d}\right)} .
\end{aligned}
$$

In the last estimate, we have used $p_{\mu}>p_{\tau}$ and the inclusion property of Triebel-Lizorkin spaces. In the 6th estimate, we used Lemma 3.4. Thus, (3.21) holds. It follows from (3.20)-(3.21) that

$$
\left\|\mathscr{M}_{\mathcal{R}}(\vec{f})\right\|_{F_{s}^{p, q}\left(\mathbb{R}^{d}\right)} \leq C \prod_{i=1}^{m}\left\|f_{i}\right\|_{F_{s}^{p_{i}, q}\left(\mathbb{R}^{d}\right)} .
$$

This completes the proof of the boundedness part.

Below we prove the continuity part. Let $f_{i, j} \rightarrow f_{i}$ in $F_{s}^{p_{i}, q}\left(\mathbb{R}^{d}\right)$ as $j \rightarrow \infty$. It is known that that $f_{i, j} \rightarrow f_{i}$ in $\dot{F}_{s}^{p_{i}, q}\left(\mathbb{R}^{d}\right)$ and in $L^{p_{i}}\left(\mathbb{R}^{d}\right)$ as $j \rightarrow \infty$. By $(3.5)$, it follows that $\mathscr{M}_{\mathcal{R}}\left(\vec{f}_{j}\right) \rightarrow \mathscr{M}_{\mathcal{R}}(\vec{f})$ in $L^{p}\left(\mathbb{R}^{d}\right)$ as $j \rightarrow \infty$. Therefore, it suffices to show that $\mathscr{M}_{\mathcal{R}}\left(\vec{f}_{j}\right) \rightarrow \mathscr{M}_{\mathcal{R}}(\vec{f})$ in $\dot{F}_{s}^{p, q}\left(\mathbb{R}^{d}\right)$ as $j \rightarrow \infty$. Again, we will prove this claim by contradiction. Without loss of generality we may assume that, for every $j$, there exists $c>0$ such that

$$
\left\|\mathscr{M}_{\mathcal{R}}\left(\vec{f}_{j}\right)-\mathscr{M}_{\mathcal{R}}(\vec{f})\right\|_{\dot{F}_{s}^{p, q}\left(\mathbb{R}^{d}\right)}>c .
$$

For a measurable function $g: \mathbb{R}^{d} \times \mathbb{Z} \times \mathfrak{R}_{d} \rightarrow \mathbb{R}$, we define

$$
\|g\|_{E_{p, q}^{s}}:=\left(\int_{\mathbb{R}^{d}}\left(\sum_{k \in \mathbb{Z}} 2^{k s q}\left(\int_{\mathfrak{R}_{d}}|g(x, k, \zeta)| d \zeta\right)^{q}\right)^{p / q} d x\right)^{1 / p} .
$$

By Lemma 3.1, we see that if $1 \leq r<\min (p, q)$, then $\|f\|_{\dot{F}_{s}^{p, q}\left(\mathbb{R}^{d}\right)} \sim\left\|\Delta_{2^{-k} \zeta} f\right\|_{E_{p, q}^{s}}$. By (3.6) and (3.8), we get

$$
\begin{aligned}
& \left|\Delta_{2^{-k} \zeta}\left(\mathscr{M}_{\mathcal{R}}\left(\vec{f}_{j}\right)-\mathscr{M}_{\mathcal{R}}(\vec{f})\right)\right| \\
& \leq \sum_{l=1}^{m}\left(\sum_{\mu=1}^{l-1} \mathscr{M}_{\mathcal{R}}\left(I_{\mu, j}^{\vec{k}, \zeta}\right)+\sum_{\nu=l+1}^{m} \mathscr{M}_{\mathcal{R}}\left(J_{\nu, j}^{\vec{k}, \zeta}\right)+\mathscr{M}_{\mathcal{R}}\left(K_{i, j}^{\vec{k}, \zeta}\right)\right)+2 \sum_{l=1}^{m} \mathscr{M}_{\mathcal{R}}\left(\vec{f}_{l}^{k, \zeta}\right),
\end{aligned}
$$

where $\vec{f}_{l}^{k, \zeta}$ is given as in (3.19) and $I_{\mu, j}^{\vec{k}, \zeta}, J_{\nu, j}^{\vec{k}, \zeta}$ and $K_{i, j}^{\vec{k}, \zeta}$ are given as in (3.8).

Notice that

$$
\begin{aligned}
\mathscr{M}_{\mathcal{R}}\left(I_{\mu, j}^{\overrightarrow{k, \zeta}}\right) \leq & \prod_{i=1}^{\mu-1} \mathcal{M}_{\mathcal{R}} f_{i} \mathcal{M}_{\mathcal{R}}\left(f_{\mu, j}-f_{\mu}\right) \prod_{\ell=\mu+1}^{l-1} \mathcal{M}_{\mathcal{R}} f_{\ell, j} \mathcal{M}_{\mathcal{R}}\left(\Delta_{2^{-k} \zeta} f_{l, j}\right) \prod_{w=l+1}^{m} \mathcal{M}_{\mathcal{R}} f_{w, j}^{k, \zeta} \\
= & \prod_{i=1}^{\mu-1} \mathcal{M}_{\mathcal{R}} f_{i} \mathcal{M}_{\mathcal{R}}\left(f_{\mu, j}-f_{\mu}\right) \prod_{\ell=\mu+1}^{l-1} \mathcal{M}_{\mathcal{R}} f_{\ell, j} \mathcal{M}_{\mathcal{R}}\left(\Delta_{2^{-k} \zeta} f_{l, j}\right) \\
& \times \prod_{w=l+1}^{m} \mathcal{M}_{\mathcal{R}}\left(\Delta_{2^{-k} \zeta} f_{w, j}+f_{w, j}\right)
\end{aligned}
$$




$$
\begin{aligned}
\leq & \prod_{i=1}^{\mu-1} \mathcal{M}_{\mathcal{R}} f_{i} \mathcal{M}_{\mathcal{R}}\left(f_{\mu, j}-f_{\mu}\right) \prod_{\ell=\mu+1}^{l-1} \mathcal{M}_{\mathcal{R}} f_{\ell, j} \mathcal{M}_{\mathcal{R}}\left(\Delta_{2^{-k} \zeta} f_{l, j}\right) \\
& \times \prod_{w=l+1}^{m}\left(\mathcal{M}_{\mathcal{R}}\left(\Delta_{2^{-k} \zeta} f_{w, j}\right)+\mathcal{M}_{\mathcal{R}} f_{w, j}\right) .
\end{aligned}
$$

This together with the arguments similar to those used in deriving (3.21) yields that

$$
\left\|\mathscr{M}_{\mathcal{R}}\left(I_{\mu, j}^{\vec{k}, \zeta}\right)\right\|_{E_{p, q}^{s}} \lesssim\left\|f_{\mu, j}-f_{\mu}\right\|_{F_{s}^{p_{\mu}, q}\left(\mathbb{R}^{d}\right)} \prod_{i=1}^{\mu-1}\left\|f_{i}\right\|_{F_{s}^{p_{i}, q}\left(\mathbb{R}^{d}\right)} \prod_{w=\mu+1}^{m}\left\|f_{w, j}\right\|_{F_{s}^{p_{w}, q}\left(\mathbb{R}^{d}\right)}
$$

Similarly, we can conclude that

$$
\begin{gathered}
\left\|\mathscr{M}_{\mathcal{R}}\left(J_{\nu, j}^{\vec{k}, \zeta}\right)\right\|_{E_{p, q}^{s}} \lesssim\left\|f_{\nu, j}-f_{\nu}\right\|_{F_{s}^{p_{\nu}, q}\left(\mathbb{R}^{d}\right)} \prod_{i=1}^{\nu-1}\left\|f_{i}\right\|_{F_{s}^{p_{i}, q}\left(\mathbb{R}^{d}\right)} \prod_{w=\nu+1}^{m}\left\|f_{w, j}\right\|_{F_{s}^{p_{w}, q}\left(\mathbb{R}^{d}\right)} ; \\
\left\|\mathscr{M}_{\mathcal{R}}\left(K_{i, j}^{\vec{k}, \zeta}\right)\right\|_{E_{p, q}^{s}} \lesssim\left\|f_{l, j}-f_{l}\right\|_{F_{s}^{p_{l}, q}\left(\mathbb{R}^{d}\right)} \prod_{\ell=1}^{l-1}\left\|f_{\ell}\right\|_{F_{s}^{p_{\ell}, q}\left(\mathbb{R}^{d}\right)} \prod_{w=l+1}^{m}\left\|f_{w, j}\right\|_{F_{s}^{p_{w}, q}\left(\mathbb{R}^{d}\right)} \\
\left\|\mathscr{M}_{\mathcal{R}}\left(\vec{f}_{l}^{k, \zeta}\right)\right\|_{E_{p, q}^{s}} \lesssim \prod_{\ell=1}^{m}\left\|f_{\ell}\right\|_{F_{s}^{p_{\ell}, q}\left(\mathbb{R}^{d}\right)} .
\end{gathered}
$$

It follows from (3.24)-(3.27) that

$$
\left\|\sum_{\mu=1}^{l-1} \mathscr{M}_{\mathcal{R}}\left(I_{\mu, j}^{\overrightarrow{k, \zeta}}\right)+\sum_{\nu=l+1}^{m} \mathscr{M}_{\mathcal{R}}\left(J_{\nu, j}^{\vec{k}, \zeta}\right)+\mathscr{M}_{\mathcal{R}}\left(K_{i, j}^{\vec{k}, \zeta}\right)\right\|_{E_{p, q}^{s}} \rightarrow 0 \text { as } j \rightarrow \infty .
$$

Therefore, one can extract a subsequence, we still denote it by $j$, such that

$$
\sum_{j=1}^{\infty}\left\|\sum_{\mu=1}^{l-1} \mathscr{M}_{\mathcal{R}}\left(I_{\mu, j}^{\vec{k}, \zeta}\right)+\sum_{\nu=l+1}^{m} \mathscr{M}_{\mathcal{R}}\left(J_{\nu, j}^{\vec{k}, \zeta}\right)+\mathscr{M}_{\mathcal{R}}\left(K_{i, j}^{\vec{k}, \zeta}\right)\right\|_{E_{p, q}^{s}}<\infty
$$

Let

$$
\begin{aligned}
G(x, k, \zeta)= & \sum_{l=1}^{m} \sum_{j=1}^{\infty} \sum_{\mu=1}^{l-1} \mathscr{M}_{\mathcal{R}}\left(I_{\mu, j}^{\vec{k}, \zeta}\right)(x)+\sum_{\nu=l+1}^{m} \mathscr{M}_{\mathcal{R}}\left(J_{\nu, j}^{\vec{k}, \zeta}\right)(x) \\
& +\mathscr{M}_{\mathcal{R}}\left(K_{i, j}^{\vec{k}, \zeta}\right)(x)+2 \sum_{l=1}^{m} \mathscr{M}_{\mathcal{R}}\left(\vec{f}_{l}^{k, \zeta}\right)(x) .
\end{aligned}
$$

We get from (3.27) and (3.28) that $\|G\|_{E_{p, q}^{s}}<\infty$. Furthermore by (3.23), one obtains that

$$
\left|\Delta_{2^{-k} \zeta}\left(\mathscr{M}_{\mathcal{R}}\left(\vec{f}_{j}\right)-\mathscr{M}_{\mathcal{R}}(\vec{f})\right)(x)\right| \leq G(x, k, \zeta) \text { for every }(x, k, \zeta) \in \mathbb{R}^{d} \times \mathbb{Z} \times \Re_{d} .
$$

(3.29) together with the dominated convergence theorem leads to

$$
\int_{\mathfrak{R}_{d}}\left|\Delta_{2^{-k} \zeta}\left(\mathscr{M}_{\mathcal{R}}\left(\vec{f}_{j}\right)-\mathscr{M}_{\mathcal{R}}(\vec{f})\right)(x)\right| d \zeta \rightarrow 0 \text { as } j \rightarrow \infty \text { for every }(x, k, \zeta) \in \mathbb{R}^{d} \times \mathbb{Z} \times \mathfrak{R}_{d}
$$

Since it holds that $\|G\|_{E_{p, q}^{s}}<\infty$, we immediately deduce that

$$
\left(\sum_{k \in \mathbb{Z}} 2^{k s q}\left(\int_{\Re_{d}} G(x, k, \zeta) d \zeta\right)^{q}\right)^{1 / q}<\infty, \quad \text { for a.e. } x \in \mathbb{R}^{d}
$$


Using (3.29), we obtain

$$
\int_{\mathfrak{R}_{d}}\left|\Delta_{2^{-k} \zeta}\left(\mathscr{M}_{\mathcal{R}}\left(\vec{f}_{j}\right)-\mathscr{M}_{\mathcal{R}}(\vec{f})\right)(x)\right| d \zeta \leq \int_{\mathfrak{R}_{d}} G(x, k, \zeta) d \zeta, \quad \text { for a.e. } x \in \mathbb{R}^{d} \text { and } k \in \mathbb{Z} .
$$

(3.30)-(3.32) and the dominated convergence theorem give

$$
\left(\sum_{k \in \mathbb{Z}} 2^{k s q}\left(\int_{\mathfrak{R}_{d}}\left|\Delta_{2^{-k} \zeta}\left(\mathscr{M}_{\mathcal{R}}\left(\vec{f}_{j}\right)-\mathscr{M}_{\mathcal{R}}(\vec{f})\right)(x)\right| d \zeta\right)^{q}\right)^{1 / q} \rightarrow 0 \text { as } j \rightarrow \infty \text {, for a.e. } x \in \mathbb{R}^{d}
$$

By 3.29 again, for a.e. $x \in \mathbb{R}^{d}$, it is true that

$$
\left(\sum_{k \in \mathbb{Z}} 2^{k s q}\left(\int_{\mathfrak{R}_{d}}\left|\Delta_{2^{-k} \zeta}\left(\mathscr{M}_{\mathcal{R}}\left(\vec{f}_{j}\right)-\mathscr{M}_{\mathcal{R}}(\vec{f})\right)(x)\right| d \zeta\right)^{q}\right)^{1 / q} \leq\left(\sum_{k \in \mathbb{Z}} 2^{k s q}\left(\int_{\mathfrak{R}_{d}} G(x, k, \zeta) d \zeta\right)^{q}\right)^{1 / q}
$$

It follows from (3.33)-(3.34), $\|G\|_{E_{p, q}^{s}}<\infty$ and the dominated convergence theorem that

$$
\lim _{j \rightarrow \infty}\left\|\Delta_{2^{-k} \zeta}\left(\mathscr{M}_{\mathcal{R}}\left(\vec{f}_{j}\right)-\mathscr{M}_{\mathcal{R}}(\vec{f})\right)\right\|_{E_{p, q}^{s}}=0,
$$

which yields $\left\|\mathscr{M}_{\mathcal{R}}\left(\vec{f}_{j}\right)-\mathscr{M}_{\mathcal{R}}(\vec{f})\right\|_{\dot{F}_{s}^{p, q}\left(\mathbb{R}^{d}\right)} \rightarrow 0$ as $j \rightarrow \infty$ and leads to a contradiction.

\section{Property of $p$-QUASICONTINUITY}

Proof. We will divide the proof of Theorem 1.5 into three steps.

Step 1: A weak type inequality for the Sobolev capacity. Let us begin with a capacity inequality that can be used in studying the pointwise behaviour of Sobolev functions by the standard methods (see [15]). Let $\vec{f}=\left(f_{1}, \ldots, f_{m}\right)$ with each $f_{i} \in W^{1, p_{i}}\left(\mathbb{R}^{d}\right)$ for $1<p_{i}<\infty$. Let $1<p<\infty$ and $1 / p=\sum_{i=1}^{m} 1 / p_{i}$. For $\lambda>0$, we set

$$
O_{\lambda}=\left\{x \in \mathbb{R}^{d} ; \mathscr{M}_{\mathcal{R}}(\vec{f})(x)>\lambda\right\} .
$$

Note that $O_{\lambda}$ is an open set. We get from Theorem 1.1 that

$$
\begin{aligned}
C_{p}\left(O_{\lambda}\right)^{1 / p} & \leq \frac{1}{\lambda}\left(\int_{\mathbb{R}^{d}}\left(\left|\mathscr{M}_{\mathcal{R}}(\vec{f})(x)\right|^{p}+\left|\nabla \mathscr{M}_{\mathcal{R}}(\vec{f})(x)\right|^{p}\right) d x\right)^{1 / p} \\
& \leq \frac{1}{\lambda}\left\|\mathscr{M}_{\mathcal{R}}(\vec{f})\right\|_{1, p} \lesssim_{m, d, p_{1}, \ldots, p_{m}} \prod_{i=1}^{m} \frac{\left\|f_{i}\right\|_{1, p_{i}}}{\lambda} .
\end{aligned}
$$

Step 2: The continuity of $\mathscr{M}_{\mathcal{R}}(\vec{f})$. To prove the $p$-quasicontinuity of $\mathscr{M}_{\mathcal{R}}(\vec{f})$, we first prove that $\mathscr{M}_{\mathcal{R}}(\vec{f}) \in \mathcal{C}\left(\mathbb{R}^{d}\right)$ if $\vec{f}=\left(f_{1}, \ldots, f_{m}\right)$ with each $f_{i} \in \mathcal{C}_{0}^{\infty}\left(\mathbb{R}^{d}\right)$. We can write

$$
\mathscr{M}_{\mathcal{R}}(\vec{f})(x)=\sup _{\vec{r} \in \mathbb{R}_{+}^{2 d}} \prod_{i=1}^{m} \frac{1}{\left|E_{\vec{r}}(x)\right|} \int_{E_{\vec{r}}(x)}\left|f_{i}(y)\right| d y,
$$

where $\vec{r}=\left(r_{1}^{-}, \ldots, r_{d}^{-} ; r_{1}^{+}, \ldots, r_{d}^{+}\right)$and $E_{\vec{r}}(x)=\left(x-r_{1}^{-}, x+r_{1}^{+}\right) \times \cdots \times\left(x-r_{d}^{-}, x+r_{d}^{+}\right)$. For fixed $x, h \in \mathbb{R}^{d}$, we have

$$
\begin{aligned}
& \left|\mathscr{M}_{\mathcal{R}}(\vec{f})(x+h)-\mathscr{M}_{\mathcal{R}}(\vec{f})(x)\right| \\
& \leq \sum_{i=1}^{m} \sup _{\vec{r} \in \mathbb{R}_{+}^{2 d}} \frac{1}{\left|E_{\vec{r}}(x)\right|^{m}} \int_{E_{\vec{r}}(x)}\left|f_{i}(y+h)-f_{i}(y)\right| d y \\
& \quad \times\left(\prod_{\mu=1}^{i-1} \int_{E_{\vec{r}}(x)}\left|f_{\mu}(y)\right| d y\right)\left(\prod_{\nu=i+1}^{m} \int_{E_{\vec{r}}(x+h)}\left|f_{\nu}(y)\right| d y\right) .
\end{aligned}
$$


For fixed $\vec{r} \in \mathbb{R}_{+}^{2 d}$ and $i=1, \ldots, m$, by Hölder's inequality, we obtain

$$
\begin{aligned}
& \frac{1}{\left|E_{\vec{r}}(x)\right|^{m}} \int_{E_{\vec{r}}(x)}\left|f_{i}(y+h)-f_{i}(y)\right| d y\left(\prod_{\mu=1}^{i-1} \int_{E_{\vec{r}}(x)}\left|f_{\mu}(y)\right| d y\right)\left(\prod_{\nu=i+1}^{m} \int_{E_{\vec{r}}(x+h)}\left|f_{\nu}(y)\right| d y\right) \\
& \leq 2\left|E_{\vec{r}}(x)\right|^{-1 / p} \prod_{i=1}^{m}\left\|f_{i}\right\|_{L^{p_{i}\left(\mathbb{R}^{d}\right)}} .
\end{aligned}
$$

It follows that given $\epsilon>0$, there exists a constant $0<\delta_{\epsilon}<+\infty$ such that

$$
\frac{1}{\left|E_{\vec{r}}(x)\right|^{m}} \int_{E_{\vec{r}}(x)}\left|f_{i}(y+h)-f_{i}(y)\right| d y\left(\prod_{\mu=1}^{i-1} \int_{E_{\vec{r}}(x)}\left|f_{\mu}(y)\right| d y\right)\left(\prod_{\nu=i+1}^{m} \int_{E_{\vec{r}}(x+h)}\left|f_{\nu}(y)\right| d y\right)<\epsilon,
$$

when $\left|E_{\vec{r}}(x)\right|>\delta_{\epsilon}$. On the other hand, for any $x, h \in \mathbb{R}^{d}$ and $\vec{r} \in \mathbb{R}_{+}^{2 d}$ with $\left|E_{\vec{r}}(x)\right| \leq \delta_{\epsilon}$, by the mean value theorem for differentials, we have

$$
\frac{1}{\left|E_{\vec{r}}(x)\right|} \int_{E_{\vec{r}}(x)}\left|f_{i}(y+h)-f_{i}(y)\right| d y \leq C\left(f_{i}\right)|h|
$$

and there exists $M_{i}>0$ such that $\left|f_{i}(x)\right| \leq M_{i}$ for all $x \in \mathbb{R}^{d}$ and $i=1, \ldots, m$. Then we have

$$
\begin{aligned}
& \frac{1}{\left|E_{\vec{r}}(x)\right|^{m}} \int_{E_{\vec{r}}(x)}\left|f_{i}(y+h)-f_{i}(y)\right| d y\left(\prod_{\mu=1}^{i-1} \int_{E_{\vec{r}}(x)}\left|f_{\mu}(y)\right| d y\right)\left(\prod_{\nu=i+1}^{m} \int_{E_{\vec{r}}(x+h)}\left|f_{\nu}(y)\right| d y\right) \\
& \leq C\left(f_{i}\right) \prod_{\mu \neq i, 1 \leq \mu \leq m} M_{\mu}|h| .
\end{aligned}
$$

Therefore, for the above $\epsilon>0$ and fixed $x \in \mathbb{R}^{d}$, there exists $\gamma=\gamma(\epsilon)>0$, if $|h|<\gamma$, then

$$
\left|\mathscr{M}_{\mathcal{R}}(\vec{f})(x+h)-\mathscr{M}_{\mathcal{R}}(\vec{f})(x)\right| \leq C(\vec{f}) \epsilon .
$$

Thus, it holds that $\mathscr{M}_{\mathcal{R}}(\vec{f}) \in \mathcal{C}\left(\mathbb{R}^{d}\right)$.

Step 3: The p-quasicontinuity of $\mathscr{M}_{\mathcal{R}}(\vec{f})$. Suppose that $f_{i} \in W^{1, p_{i}}\left(\mathbb{R}^{d}\right)$, we can choose a sequence of functions $\left\{f_{i, k}\right\}_{k \geq 1} \subset \mathcal{C}_{0}^{\infty}\left(\mathbb{R}^{d}\right)$ such that $f_{i, k} \rightarrow f_{i}$ in $W^{1, p_{i}}\left(\mathbb{R}^{d}\right)$. This yields that there exists a large $K_{0} \in \mathbb{N}$ such that

$$
\left\|f_{i, k}-f_{i}\right\|_{1, p_{i}} \leq 2^{-2 k}, \quad \forall k \geq K_{0} \text { and } 1 \leq i \leq m .
$$

Fix $k \geq K_{0}$. Let $\vec{f}_{k}=\left(f_{1, k}, \ldots, f_{m, k}\right)$ and

$$
E_{k}=\left\{x \in \mathbb{R}^{d}:\left|\mathscr{M}_{\mathcal{R}}\left(\vec{f}_{k}\right)(x)-\mathscr{M}_{\mathcal{R}}(\vec{f})(x)\right|>2^{-k}\right\} .
$$

By (2.6), we have

$$
\left|\mathscr{M}_{\mathcal{R}}\left(\vec{f}_{k}\right)(x)-\mathscr{M}_{\mathcal{R}}(\vec{f})(x)\right| \leq \sum_{l=1}^{m} \mathscr{M}_{\mathcal{R}}\left(\vec{F}_{k}^{l}\right)(x),
$$

where $\vec{F}_{k}^{l}(x)=\left(f_{1}, \ldots, f_{l-1}, f_{l, k}-f_{l}, f_{l+1, k}, \ldots, f_{m, k}\right)$. Then, by (4.1)-(4.3), we have

$$
\begin{aligned}
\left(C_{p}\left(E_{k}\right)\right)^{1 / p} & \lesssim_{m, d, p_{1}, \ldots, p_{m}} 2^{k} \sum_{l=1}^{m} \prod_{\mu=1}^{l-1}\left\|f_{\mu}\right\|_{1, p_{\mu}}\left\|f_{l, k}-f_{l}\right\|_{1, p_{l}} \prod_{\nu=l+1}^{m}\left\|f_{\nu, k}\right\|_{1, p_{\nu}} \\
& \lesssim_{m, d, p_{1}, \ldots, p_{m}} 2^{-k} .
\end{aligned}
$$

Let $G_{k}=\bigcup_{i=k}^{\infty} E_{i}$ with $k \geq K_{0}$. Then by subadditivity and (4.4), it holds that

$$
C_{p}\left(G_{k}\right) \leq \sum_{i=k}^{\infty} C_{p}\left(E_{i}\right) \lesssim_{m, d, p_{1}, \ldots, p_{m}} \sum_{i=k}^{\infty} 2^{-i p} \lesssim_{m, d, p_{1}, \ldots, p_{m}} \frac{2^{(1-k) p}}{2^{p}-1}, \quad \forall k \geq K_{0},
$$


which leads to $\lim _{k \rightarrow \infty} C_{p}\left(G_{k}\right)=0$. On the other hand, for $x \in \mathbb{R}^{d} \backslash G_{k}$,

$$
\left|\mathscr{M}_{\mathcal{R}}\left(\vec{f}_{k}\right)(x)-\mathscr{M}_{\mathcal{R}}(\vec{f})(x)\right| \leq 2^{-k} \quad \forall k \geq K_{0} .
$$

This implies that $\left\{\mathscr{M}_{\mathcal{R}}\left(\vec{f}_{k}\right)\right\}$ converges to $\mathscr{M}_{\mathcal{R}}(\vec{f})$ uniformly in $\mathbb{R}^{d} \backslash G_{k}$. By Step 2, we see that $\mathscr{M}_{\mathcal{R}}\left(\vec{f}_{k}\right) \in \mathcal{C}\left(\mathbb{R}^{d}\right)$. It follows that $\mathscr{M}_{\mathcal{R}}(\vec{f})$ is continuous in $\mathbb{R}^{d} \backslash G_{k}$. We notice that $\mathscr{M}_{\mathcal{R}}\left(\vec{f}_{K_{0}}\right)(x)<$ $\infty$ for all $x \in \mathbb{R}^{d}$. This together with (4.5) implies that $\mathscr{M}_{\mathcal{R}}(\vec{f})$ is finite in $\mathbb{R}^{d} \backslash G_{k}$. Hence, $\mathscr{M}_{\mathcal{R}}(\vec{f})$ is $q$-quasicontinuous.

\section{Approximate differentiability of $\mathscr{M}_{\mathcal{R}}$}

This section is devoted to proving Theorem 1.6. Let us recall some definitions and present some useful lemmas.

Let $f$ be a real-valued function defined on a set $E \subset \mathbb{R}^{d}$. We say that $f$ is approximately differentiable at $x_{0} \in E$ if there is a vector $L=\left(L_{1}, L_{2}, \ldots, L_{d}\right) \in \mathbb{R}^{d}$ such that for any $\epsilon>0$ the set

$$
A_{\epsilon}=\left\{x \in \mathbb{R}^{d}: \frac{\left|f(x)-f\left(x_{0}\right)-L\left(x-x_{0}\right)\right|}{\left|x-x_{0}\right|}<\epsilon\right\}
$$

has $x_{0}$ as a density point. If this is the case, then $x_{0}$ is a density point of $E$ and $L$ is uniquely determined. The vector $L$ is called the approximate differential of $f$ at $x_{0}$ and is denoted by $\nabla f\left(x_{0}\right)$. Note that every function $f \in W^{1,1}\left(\mathbb{R}^{d}\right)$ is approximately differentiable a.e. It was pointed out in [22] that $M f$ is approximately differentiable a.e. under the assumption that $f \in W^{1,1}\left(\mathbb{R}^{d}\right)$. However, it is unknown that whether $f \in W^{1,1}\left(\mathbb{R}^{d}\right)$ implies the weak differentiability of $M f$ when $d \geq 2$. The relationship between approximate differentiability and weak differentiability is still not clear.

To prove Theorem 1.6, we need the following lemma, which provides several characterizations of a.e. approximate differentiability of a function.

Lemma 5.1. ([54]) Let $f: E \rightarrow \mathbb{R}$ be measurable, $E \subset \mathbb{R}^{d}$. Then the following conditions are equivalent:

(i) $f$ is approximately differentiable a.e.

(ii) For any $\epsilon>0$, there is a closed set $F \subset E$ and a locally Lipschitz function $g: \mathbb{R}^{d} \rightarrow \mathbb{R}$ such that $f=g$ on $x \in F$ and $|E \backslash F|<\epsilon$.

(iii) For any $\epsilon>0$, there is a closed set $F \subset E$ and a function $g \in \mathcal{C}^{1}\left(\mathbb{R}^{d}\right)$ such that $f=g$ on $x \in F$ and $|E \backslash F|<\epsilon$.

Lemma 5.2. Let $\vec{f}=\left(f_{1}, \ldots, f_{m}\right)$ with each $f_{j} \in L^{1}\left(\mathbb{R}^{d}\right)$. Let $\vec{\varepsilon}=\left(\varepsilon_{1}, \ldots, \varepsilon_{d}\right)$ with each $\varepsilon_{i}>0$. The truncated multilinear strong maximal operator $\mathscr{M}_{\mathcal{R}}^{\vec{\varepsilon}}$ is defined by

$$
\mathscr{M}_{\mathcal{R}}^{\overrightarrow{\mathcal{R}}}(\vec{f})(x)=\sup _{\substack{\left(r_{1}^{-}, \ldots, r_{d}^{-} ; r_{1}^{+}, \ldots, r_{d}^{+}\right) \in \mathbb{R}_{+}^{2 d} \\ r_{i}^{+}+r_{i}^{-} \geq \varepsilon_{i}, i=1,2, \ldots, d}} \prod_{i=1}^{m} \frac{1}{\left|E_{\vec{r}}(x)\right|} \int_{E_{\vec{r}}(x)}\left|f_{i}(y)\right| d y,
$$

where $x=\left(x_{1}, \ldots, x_{d}\right), \vec{r}=\left(r_{1}^{-}, \ldots, r_{d}^{-} ; r_{1}^{+}, \ldots, r_{d}^{+}\right)$and $E_{\vec{r}}(x)=\left(x_{1}-r_{1}^{-}, x_{1}+r_{1}^{+}\right) \times \cdots \times\left(x_{d}-\right.$ $\left.r_{d}^{-}, x_{d}+r_{d}^{+}\right)$. Then $\mathscr{M}_{\mathcal{R}}^{\vec{\varepsilon}}(\vec{f})$ is Lipschitz continuous for every $\vec{\varepsilon} \in \mathbb{R}_{+}^{d}$.

Proof. Fix $\vec{\varepsilon}=\left(\varepsilon_{1}, \ldots, \varepsilon_{d}\right) \in \mathbb{R}_{+}^{d}$. We set $\varepsilon_{0}=\min _{1 \leq i \leq d} \varepsilon_{i}$. Fix $\vec{r}=\left(r_{1}^{-}, \ldots, r_{d}^{-} ; r_{1}^{+}, \ldots, r_{d}^{+}\right) \in$ $\mathbb{R}_{+}^{2 d}$ with $r_{i}^{+}+r_{i}^{-} \geq \varepsilon_{i}$ for $1 \leq i \leq d$. It is obvious that $r_{i}^{+}+r_{i}^{-} \geq \varepsilon_{0}$ for all $1 \leq i \leq d$.

Notice that for any $r \geq a \geq 0, b \geq 0$ and $\delta \geq 1$, it is true that

$$
\left(\frac{r}{r+b}\right)^{\delta} \geq\left(\frac{a}{a+b}\right)^{\delta} \geq 1-\delta \frac{b}{a}
$$


Let $x=\left(x_{1}, \ldots, x_{d}\right) \in \mathbb{R}^{d}$ and $y=\left(y_{1}, \ldots, y_{d}\right) \in \mathbb{R}^{d}$. We note that

$$
E_{\vec{r}}(x) \subset E_{\vec{r}^{\prime}}(y)
$$

where $\vec{r}^{\prime}=\left(r_{1}^{-}+\left|y_{1}-x_{1}\right|, \ldots, r_{d}^{-}+\left|y_{d}-x_{d}\right| ; r_{1}^{+}+\left|y_{1}-x_{1}\right|, \ldots, r_{d}^{+}+\left|y_{d}-x_{d}\right|\right)$. (5.2) gives that

$$
\left(\frac{\left|E_{\vec{r}}(x)\right|}{\left|E_{\vec{r}^{\prime}}(y)\right|}\right)^{m}=\left(\prod_{j=1}^{d} \frac{r_{j}^{-}+r_{j}^{+}}{r_{j}^{-}+r_{j}^{+}+\left|y_{j}-x_{j}\right|}\right)^{m} \geq\left(\frac{\varepsilon_{0}}{\varepsilon_{0}+|x-y|}\right)^{m d} \geq 1-\frac{m d}{\varepsilon_{0}}|x-y| .
$$

We get from (5.2) and (5.3) that

$$
\begin{aligned}
\mathscr{M}_{\mathcal{R}}^{\vec{\varepsilon}}(\vec{f})(y) & \geq \prod_{i=1}^{m} \frac{1}{\left|E_{\vec{r}^{\prime}}(y)\right|} \int_{E_{\vec{r}}(y)}\left|f_{i}(z)\right| d z \\
& \geq \prod_{i=1}^{m} \frac{\left|E_{\vec{r}}(x)\right|}{\left|E_{\vec{r}^{\prime}}(y)\right|} \frac{1}{\left|E_{\vec{r}}(x)\right|} \int_{E_{\vec{r}}(x)}\left|f_{i}(z)\right| d z \\
& \geq\left(1-\frac{m d}{\varepsilon_{0}}|x-y|\right) \prod_{i=1}^{m} \frac{1}{\left|E_{\vec{r}}(x)\right|} \int_{E_{\vec{r}}(x)}\left|f_{i}(z)\right| d z
\end{aligned}
$$

Taking the supremum over $\vec{r}=\left(r_{1}^{-}, \ldots, r_{d}^{-} ; r_{1}^{+}, \ldots, r_{d}^{+}\right) \in \mathbb{R}_{+}^{2 d}$ with $r_{i}^{+}+r_{i}^{-} \geq \varepsilon_{i}$ for $1 \leq i \leq d$, we get from (5.4) that

$$
\mathscr{M}_{\mathcal{R}}^{\vec{\varepsilon}}(\vec{f})(y) \geq\left(1-\frac{m d}{\varepsilon_{0}}|x-y|\right) \mathscr{M}_{\mathcal{R}}^{\vec{\varepsilon}}(\vec{f})(x)
$$

It follows that

$$
\mathscr{M}_{\mathcal{R}}^{\vec{\varepsilon}}(\vec{f})(x)-\mathscr{M}_{\mathcal{R}}^{\vec{\varepsilon}}(\vec{f})(y) \leq \frac{m d}{\varepsilon_{0}}|x-y| \mathscr{M}_{\mathcal{R}}^{\vec{\varepsilon}}(\vec{f})(x)
$$

Similarly, we can get

$$
\mathscr{M}_{\mathcal{R}}^{\vec{\varepsilon}}(\vec{f})(y)-\mathscr{M}_{\mathcal{R}}^{\vec{\varepsilon}}(\vec{f})(x) \leq \frac{m d}{\varepsilon_{0}}|x-y| \mathscr{M}_{\mathcal{R}}^{\vec{\varepsilon}}(\vec{f})(y)
$$

Thus, (5.5) and (5.6) imply that

$$
\left|\mathscr{M}_{\mathcal{R}}^{\vec{\varepsilon}}(\vec{f})(x)-\mathscr{M}_{\mathcal{R}}^{\vec{\varepsilon}}(\vec{f})(y)\right| \leq \frac{m d}{\varepsilon_{0}}|x-y|\left(\mathscr{M}_{\mathcal{R}}^{\vec{\varepsilon}}(\vec{f})(x)+\mathscr{M}_{\mathcal{R}}^{\vec{\varepsilon}}(\vec{f})(y)\right) \leq \frac{2 m d}{\varepsilon_{0}^{m d+1}} \prod_{j=1}^{m}\left\|f_{j}\right\|_{L^{1}\left(\mathbb{R}^{d}\right)}|x-y| .
$$

This proves Lemma 5.2 .

Proof of Theorem 1.6. Let $Z_{j}$ be the set of all Lebesgue points of $f_{j}$ and $u_{x, \vec{f}}(r)$ defined as in Section 2. We set $E_{0}=\mathbb{R}^{d} \backslash\left(\bigcap_{j=1}^{m} Z_{j}\right)$. Let $x \in \bigcap_{j=1}^{m} Z_{j}$ such that $\mathscr{M}_{\mathcal{R}}(\vec{f})(x)>u_{x, \vec{f}}(\overrightarrow{0})$ with $\overrightarrow{0} \in \mathbb{R}^{2 d}$. Since $f_{j} \in L^{1}\left(\mathbb{R}^{d}\right)$ and $\mathscr{M}_{\mathcal{R}}(\vec{f})(x)>0$, there exists a sequence $\left\{\vec{r}_{k}\right\}_{k \geq 1}$ with $\vec{r}_{k}=\left(r_{1, k}^{-}, \ldots, r_{d, k}^{-} ; r_{1, k}^{+}, \ldots, r_{d, k}^{+}\right) \in \overline{\mathbb{R}}_{+}^{2 d}$, all $r_{i, k}^{-}+r_{i, k}^{+}$are bounded such that

$$
\lim _{k \rightarrow \infty} u_{x, \vec{f}}\left(\vec{r}_{k}\right)=\mathscr{M}_{\mathcal{R}}(\vec{f})(x) .
$$

Hence there exists a subsequence $\left\{\vec{r}_{k}^{\prime}\right\}_{k \geq 1} \subset\left\{\vec{r}_{k}\right\}_{k \geq 1}$ and $\vec{r}=\left(r_{1}^{-}, \ldots, r_{d}^{-} ; r_{1}^{+}, \ldots, r_{d}^{+}\right) \in \overline{\mathbb{R}}_{+}^{2 d}$ with $r_{i}^{-}+r_{i}^{+}>0$ for all $1 \leq i \leq d$ such that $\lim _{k \rightarrow \infty} \vec{r}_{k}^{\prime}=\vec{r}$. It follows that $\mathscr{M}_{\mathcal{R}}(\vec{f})(x)=u_{x, \vec{f}}(\vec{r})$. This, of course, yields that

$$
\mathbb{R}^{d}=E_{0} \cup\left\{x \in \mathbb{R}^{d}: \mathscr{M}_{\mathcal{R}}(\vec{f})(x)=u_{x, \vec{f}}(\overrightarrow{0})\right\} \cup E,
$$


where $E=\bigcup_{k_{1}=1}^{\infty} \ldots \bigcup_{k_{d}=1}^{\infty} E_{k_{1}, \ldots, k_{d}}$ and $E_{k_{1}, \ldots, k_{d}}=\left\{x \in \mathbb{R}^{d}: \mathscr{M}_{\mathcal{R}}(\vec{f})(x)=\mathscr{M}_{\mathcal{R}}^{1 / k_{1}, \ldots, 1 / k_{d}}(\vec{f})(x)\right\}$. By Lemma 5.1, $\prod_{j=1}^{m}\left|f_{j}\right|$ is approximately differentiable a.e. Then $\mathscr{M}_{\mathcal{R}}(\vec{f})$ is approximately differentiable a.e. in the set $\left\{x \in \mathbb{R}^{2}: \mathscr{M}_{\mathcal{R}}(\vec{f})(x)=u_{x, \vec{f}}(\overrightarrow{0})\right\}$. By Lemma 5.2 we have that $\mathscr{M}_{\mathcal{R}}^{1 / k_{1}, \ldots, 1 / k_{d}}(\vec{f})$ is Lipschitz continuous for any $k_{i} \geq 1$ and $1 \leq i \leq d$. Then, for any $k_{i} \geq 1$ and $1 \leq i \leq d$, the function $\mathscr{M}_{\mathcal{R}}^{1 / k_{1}, \ldots, 1 / k_{d}}(\vec{f})$ is approximately differentiable a.e.. It follows that $\mathscr{M}_{\mathcal{R}}(\vec{f}) \chi_{E}$ is approximately differentiable a.e. Note that $\left|E_{0}\right|=0$. Therefore, $\mathscr{M}_{\mathcal{R}}(\vec{f})$ is approximately differentiable a.e. This completes the proof of Theorem 1.6.

\section{Properties of Discrete Strong maximal Functions}

This section is devoted to proving Theorem 1.7. For $a \in \mathbb{R}$ and $r>0$, we define

$$
g(a ; r)=|\{k \in \mathbb{Z} ;|k-a|<r\}| .
$$

If $a \in \mathbb{Z}$, then $g(a ; r) \geq \chi_{(0,1]}(r)+(2[r-1]+1) \chi_{(1, \infty)}$, where $[x]=\max \{k \in \mathbb{Z} ; k \leq x\}$. If $a \in \mathbb{R} \backslash \mathbb{Z}$, then there exists an integer $n_{0} \in \mathbb{Z}$ such that $\left|n_{0}-a\right| \leq 1 / 2$ and

$$
\left\{k \in \mathbb{Z} ;\left|k-n_{0}\right|<r-1 / 2\right\} \subset\{k \in \mathbb{Z} ;|k-a|<r\} .
$$

It follows that $g(a ; r) \geq \chi_{\left(\frac{1}{2}, \frac{3}{2}\right]}+(2[r-3 / 2]+1) \chi_{\left(\frac{3}{2}, \infty\right)}(r)$ for $r>1 / 2$. Specially, if there exists an integer $n_{0}$ such that $\left|n_{0}-a\right|<r$, then

$$
g(a ; r) \geq F(r):=\chi_{\left(0, \frac{3}{2}\right]}(r)+(2[r-3 / 2]+1) \chi_{\left(\frac{3}{2}, \infty\right)}(r) \quad \forall r>0 \text { and } a \in \mathbb{R} .
$$

For $\vec{r}=\left(r_{1}, \ldots, r_{d}\right) \in \mathbb{R}_{+}^{d}$ and $\vec{x}=\left(x_{1}, \ldots, x_{d}\right) \in \mathbb{R}^{d}$, it is easy to see that $N\left(R_{\vec{r}}(\vec{x})\right)=$ $\prod_{i=1}^{d} g\left(x_{i} ; r_{i}\right)$. Furthermore, if there exists $\vec{n} \in R_{\vec{r}}(\vec{x}) \cap \mathbb{Z}^{d}$, then by (6.1), it holds that

$$
N\left(R_{\vec{r}}(\vec{x})\right) \geq \prod_{i=1}^{d} F\left(r_{i}\right)
$$

We now divide the proof of Theorem 1.7 into two parts.

6.1. The boundedness part. Without loss of generality we may assume all $f_{j} \geq 0$ since $\|\nabla|f|\|_{\ell^{1}\left(\mathbb{Z}^{d}\right)} \leq\|\nabla f\|_{\ell^{1}\left(\mathbb{Z}^{d}\right)}$. For all $1 \leq l \leq d$, it suffices to show that

$$
\left\|D_{l} \mathbb{M}_{\mathcal{R}}(\vec{f})\right\|_{\ell^{1}\left(\mathbb{Z}^{d}\right)} \lesssim d, m \sum_{i=1}^{m}\left\|D_{l} f_{i}\right\|_{\ell^{1}\left(\mathbb{Z}^{d}\right)} \prod_{j \neq i, 1 \leq j \leq m}\left\|f_{j}\right\|_{\ell^{1}\left(\mathbb{Z}^{d}\right)}
$$

We only prove (6.3) for $l=d$, since the other cases are analogous. In what follows, we set $\vec{n}=\left(n^{\prime}, n_{d}\right) \in \mathbb{Z}^{d}$ with $n^{\prime}=\left(n_{1}, \ldots, n_{d-1}\right) \in \mathbb{Z}^{d-1}$. For each $n^{\prime} \in \mathbb{Z}^{d-1}$, let

$$
\begin{aligned}
& X_{n^{\prime}}^{+}=\left\{n_{d} \in \mathbb{Z}: \mathbb{M}_{\mathcal{R}}(\vec{f})\left(n^{\prime}, n_{d}+1\right) \leq \mathbb{M}_{\mathcal{R}}(\vec{f})\left(n^{\prime}, n_{d}\right)\right\}, \\
& X_{n^{\prime}}^{-}=\left\{n_{d} \in \mathbb{Z}: \mathbb{M}_{\mathcal{R}}(\vec{f})\left(n^{\prime}, n_{d}+1\right)>\mathbb{M}_{\mathcal{R}}(\vec{f})\left(n^{\prime}, n_{d}\right)\right\} .
\end{aligned}
$$

Then we can write

$$
\begin{aligned}
\left\|D_{l} \mathbb{M}_{\mathcal{R}}(\vec{f})\right\|_{\ell^{1}\left(\mathbb{Z}^{d}\right)}= & \sum_{n^{\prime} \in \mathbb{Z}^{d-1}} \sum_{n_{d} \in X_{n^{\prime}}^{+}}\left(\mathbb{M}_{\mathcal{R}}(\vec{f})\left(n^{\prime}, n_{d}\right)-\mathbb{M}_{\mathcal{R}}(\vec{f})\left(n^{\prime}, n_{d}+1\right)\right) \\
& +\sum_{n^{\prime} \in \mathbb{Z}^{d-1}} \sum_{n_{d} \in X_{n^{\prime}}^{-}}\left(\mathbb{M}_{\mathcal{R}}(\vec{f})\left(n^{\prime}, n_{d}+1\right)-\mathbb{M}_{\mathcal{R}}(\vec{f})\left(n^{\prime}, n_{d}\right)\right) .
\end{aligned}
$$


Therefore, to prove (6.3) with $l=d$, it suffices to show that

$$
\sum_{n^{\prime} \in \mathbb{Z}^{d-1}} \sum_{n_{d} \in X_{n^{\prime}}^{+}}\left(\mathbb{M}_{\mathcal{R}}(\vec{f})\left(n^{\prime}, n_{d}\right)-\mathbb{M}_{\mathcal{R}}(\vec{f})\left(n^{\prime}, n_{d}+1\right)\right) \lesssim d \sum_{i=1}^{m}\left\|D_{l} f_{i}\right\|_{\ell^{1}\left(\mathbb{Z}^{d}\right)} \prod_{j \neq i, 1 \leq j \leq m}\left\|f_{j}\right\|_{\ell^{1}\left(\mathbb{Z}^{d}\right)} ;
$$

$$
\sum_{n^{\prime} \in \mathbb{Z}^{d-1}} \sum_{n_{d} \in X_{n^{\prime}}^{-}}\left(\mathbb{M}_{\mathcal{R}}(\vec{f})\left(n^{\prime}, n_{d}+1\right)-\mathbb{M}_{\mathcal{R}}(\vec{f})\left(n^{\prime}, n_{d}\right)\right) \lesssim d \sum_{i=1}^{m}\left\|D_{l} f_{i}\right\|_{\ell^{1}\left(\mathbb{Z}^{d}\right)} \prod_{j \neq i, 1 \leq j \leq m}\left\|f_{j}\right\|_{\ell^{1}\left(\mathbb{Z}^{d}\right)} .
$$

We only prove (6.4), since (6.5) is analogous. For $\vec{r} \in \mathbb{R}_{+}^{d}$, define $A_{\vec{r}}(\vec{f}): \mathbb{R}^{d} \rightarrow \mathbb{R}$ by

$$
A_{\vec{r}}(\vec{f})(\vec{x})=\frac{1}{N\left(R_{\vec{r}}(\vec{x})\right)^{m}} \prod_{j=1}^{m} \sum_{\vec{k} \in R_{\vec{r}}(\vec{x}) \cap \mathbb{Z}^{d}} f_{j}(\vec{k}), \quad \forall \vec{x} \in \mathbb{R}^{d} .
$$

We can write

$$
\mathbb{M}_{\mathcal{R}}(\vec{f})(\vec{n})=\sup _{\substack{\vec{r} \in \mathbb{R}_{+}^{d} \\ \vec{x} \in \mathbb{R}^{d}, \vec{n} \in R_{\vec{r}}(\vec{x})}} A_{\vec{r}}(\vec{f})(\vec{x}), \quad \forall \vec{n} \in \mathbb{Z}^{d} .
$$

Lemma 6.1. Let $\vec{f}=\left(f_{1}, \ldots, f_{m}\right)$ with each $f_{j} \in \ell^{1}\left(\mathbb{Z}^{d}\right)$. Then for any $\vec{n} \in \mathbb{Z}^{d}, \mathbb{M}_{\mathcal{R}}(\vec{f})(\vec{n})$ is attained for some $R$ with $\vec{n} \in R \in \mathcal{R}$.

Proof. Fix $\vec{n} \in \mathbb{Z}^{d}$. If $\mathbb{M}_{\mathcal{R}}(\vec{f})(\vec{n})=0$, then all $f_{j} \equiv 0$. For any $R$ with $\vec{n} \in R \in \mathcal{R}$, it suffices to show that

$$
\mathbb{M}_{\mathcal{R}}(\vec{f})(\vec{n})=\frac{1}{N(R)^{m}} \prod_{i=1}^{m} \sum_{\vec{k} \in R \cap \mathbb{Z}^{d}}\left|f_{i}(\vec{k})\right|=0 .
$$

If $\mathbb{M}_{\mathcal{R}}(\vec{f})(\vec{n})>0$. Suppose that $\mathbb{M}_{\mathcal{R}}(\vec{f})(\vec{n})$ is not attained for $R$ with $\vec{n} \in R \in \mathcal{R}$. Let $\left\{r_{k}\right\}_{k \geq 1}$ be an increasing sequence of positive numbers with $\lim _{k \rightarrow \infty} r_{k}=\infty$. By the definition of $\mathbb{M}_{\mathcal{R}}(\vec{f})$ and our assumption, we have

$$
\mathbb{M}_{\mathcal{R}}(\vec{f})(\vec{n})=\sup _{\substack{\vec{n} \in R \in \mathcal{R} \\ N(R) \geq r_{k}}} \frac{1}{N(R)^{m}} \prod_{i=1}^{m} \sum_{\vec{k} \in R \cap \mathbb{Z}^{d}}\left|f_{i}(\vec{k})\right|, \quad \forall k \geq 1 .
$$

It follows that

$$
\mathbb{M}_{\mathcal{R}}(\vec{f})(\vec{n}) \leq \frac{1}{r_{k}^{m}} \prod_{i=1}^{m}\left\|f_{i}\right\|_{\ell^{1}(\mathbb{Z})}, \quad \forall k \geq 1 .
$$

Let $k \rightarrow \infty$, we obtain $\widetilde{\mathbb{M}}_{\mathcal{R}}(\vec{f})(\vec{n})=0$, which is a contradiction. Thus, $\mathbb{M}_{\mathcal{R}}(\vec{f})(\vec{n})$ is attained for some $R$ with $\vec{n} \in R \in \mathcal{R}$.

Since all $f_{j} \in \ell^{1}\left(\mathbb{Z}^{d}\right)$, by Lemma 6.1, for any $\left(n^{\prime}, n_{d}\right) \in \mathbb{Z}^{d}$, there exist $\vec{x} \in \mathbb{R}^{d}$ and $\vec{r}\left(n^{\prime}, n_{d}\right) \in$ $\mathbb{R}_{+}^{d}$ such that $\left(n^{\prime}, n_{d}\right) \in R_{\vec{r}\left(n^{\prime}, n_{d}\right)}(\vec{x})$ and $\mathbb{M}_{\mathcal{R}}(\vec{f})\left(n^{\prime}, n_{d}\right)=A_{\vec{r}\left(n^{\prime}, n_{d}\right)}(\vec{f})(\vec{x})$. Then

$$
\mathbb{M}_{\mathcal{R}}(\vec{f})\left(n^{\prime}, n_{d}\right)-\mathbb{M}_{\mathcal{R}}(\vec{f})\left(n^{\prime}, n_{d}+1\right) \leq A_{\vec{r}\left(n^{\prime}, n_{d}\right)}(\vec{f})(\vec{x})-A_{\vec{r}\left(n^{\prime}, n_{d}\right)}(\vec{f})\left(\vec{x}+\vec{e}_{d}\right) .
$$

For convenience, we set $\vec{r}\left(n^{\prime}, n_{d}\right)=\left(r_{1}\left(n^{\prime}, n_{d}\right), \ldots, r_{d}\left(n^{\prime}, n_{d}\right)\right)$. Note that $\left(n^{\prime}, n_{d}\right) \in R_{\vec{r}\left(n^{\prime}, n_{d}\right)}(\vec{x})$ and $R_{\vec{r}\left(n^{\prime}, n_{d}\right)}(\vec{x}) \subset R_{2 \vec{r}\left(n^{\prime}, n_{d}\right)}\left(n^{\prime}, n_{d}\right)$. These facts together with (6.2) yields that 


$$
\begin{aligned}
& A_{\vec{r}\left(n^{\prime}, n_{d}\right)}(\vec{f})(\vec{x})-A_{\vec{r}\left(n^{\prime}, n_{d}\right)}(\vec{f})\left(\vec{x}+\vec{e}_{d}\right) \\
& \leq \prod_{i=1}^{d} \frac{1}{F\left(r_{i}\left(n^{\prime}, n_{d}\right)\right)^{m}} \sum_{\mu=1}^{m}\left|\sum_{\vec{k} \in R_{\vec{r}\left(n^{\prime}, n_{d}\right)}(\vec{x}) \cap \mathbb{Z}^{d}} f_{\mu}(\vec{k})-\sum_{\vec{k} \in R_{\vec{r}\left(n^{\prime}, n_{d}\right)}\left(\vec{x}+\vec{e}_{d}\right) \cap \mathbb{Z}^{d}} f_{\mu}(\vec{k})\right| \\
& \quad \times\left(\prod_{i=1}^{\mu-1} \sum_{\vec{k} \in R_{\vec{r}\left(n^{\prime}, n_{d}\right)}(\vec{x}) \cap \mathbb{Z}^{d}} f_{i}(\vec{k})\right)\left(\prod_{\nu=\mu+1}^{m} \sum_{\vec{k} \in R_{\vec{r}\left(n^{\prime}, n_{d}\right)}\left(\vec{x}+\vec{e}_{d}\right) \cap \mathbb{Z}^{d}} f_{\nu}(\vec{k})\right) . \\
& \leq \sum_{\mu=1}^{m} \prod_{\nu \neq \mu, 1 \leq \nu \leq m}\left\|f_{\nu}\right\|_{\ell^{1}\left(\mathbb{Z}^{d}\right)} \prod_{i=1}^{d} \frac{1}{F\left(r_{i}\left(n^{\prime}, n_{d}\right)\right)^{m}} \sum_{\vec{k} \in R_{2 \vec{r}\left(n^{\prime}, n_{d}\right)}\left(n^{\prime}, n_{d}\right) \cap \mathbb{Z}^{d}}\left|D_{d} f_{\mu}(\vec{k})\right| .
\end{aligned}
$$

Therefore, $\mathbb{M}_{\mathcal{R}}(\vec{f})\left(n^{\prime}, n_{d}\right)-\mathbb{M}_{\mathcal{R}}(\vec{f})\left(n^{\prime}, n_{d}+1\right)$ can be controlled by

$$
\sum_{\mu=1}^{m} \prod_{\nu \neq \mu, 1 \leq \nu \leq m}\left\|f_{\nu}\right\|_{\ell^{1}\left(\mathbb{Z}^{d}\right)} \prod_{i=1}^{d} \frac{1}{F\left(r_{i}\left(n^{\prime}, n_{d}\right)\right)^{m}} \sum_{\vec{k} \in R_{2 \vec{r}\left(n^{\prime}, n_{d}\right)}\left(n^{\prime}, n_{d}\right) \cap \mathbb{Z}^{d}}\left|D_{d} f_{\mu}(\vec{k})\right| .
$$

It follows that

$$
\begin{aligned}
& \sum_{n^{\prime} \in \mathbb{Z}^{d-1}} \sum_{n_{d} \in X_{n^{\prime}}^{+}}\left(\mathbb{M}_{\mathcal{R}} f\left(n^{\prime}, n_{d}\right)-\mathbb{M}_{\mathcal{R}} f\left(n^{\prime}, n_{d}+1\right)\right) \\
\leq & \sum_{\mu=1}^{m} \prod_{\nu \neq \mu, 1 \leq \nu \leq m}\left\|f_{\nu}\right\|_{\ell^{1}\left(\mathbb{Z}^{d}\right)} \\
& \times\left(\sum_{n^{\prime} \in \mathbb{Z}^{d-1}} \sum_{n_{d} \in X_{n^{\prime}}^{+}} \prod_{i=1}^{d} \frac{1}{\left(F\left(r_{i}\left(n^{\prime}, n_{d}\right)\right)\right)^{m}} \sum_{\vec{k} \in R_{2 \vec{r}\left(n^{\prime}, n_{d}\right)}\left(n^{\prime}, n_{d}\right) \cap \mathbb{Z}^{d}}\left|D_{d} f_{\mu}(\vec{k})\right|\right) .
\end{aligned}
$$

By direct calculations, we obtain

$$
\begin{aligned}
& \sum_{n^{\prime} \in \mathbb{Z}^{d-1}} \sum_{n_{d} \in X_{n^{\prime}}^{+}} \prod_{i=1}^{d} \frac{1}{\left(F\left(r_{i}\left(n^{\prime}, n_{d}\right)\right)\right)^{m}} \sum_{\vec{k} \in R_{2 \vec{r}\left(n^{\prime}, n_{d}\right)}\left(n^{\prime}, n_{d}\right) \cap \mathbb{Z}^{d}}\left|D_{d} f_{\mu}(\vec{k})\right| \\
& \leq \sum_{\left(k_{1}, \ldots, k_{d}\right) \in \mathbb{Z}^{d}}\left|D_{d} f_{\mu}\left(k_{1}, \ldots, k_{d}\right)\right| \prod_{i=1}^{d}\left(\sum_{n_{i} \in \mathbb{Z}} \frac{1}{\left(F\left(r_{i}\left(n^{\prime}, n_{d}\right)\right)\right)^{m}} \chi_{\left\{\left|k_{i}-n_{i}\right|<2 r_{i}\left(n^{\prime}, n_{d}\right)\right\}}\right) .
\end{aligned}
$$

For fixed $1 \leq i \leq d$ and $k_{i} \in \mathbb{Z}$, we have

$$
\sum_{n_{i} \in \mathbb{Z}} \frac{1}{\left(F\left(r_{i}\left(n^{\prime}, n_{d}\right)\right)^{m}\right.} \chi_{\left\{\left|k_{i}-n_{i}\right|<2 r_{i}\left(n^{\prime}, n_{d}\right)\right\}} \leq 9+\sum_{n_{i} \in \mathbb{Z}} \frac{1}{\left(2\left[\frac{\left|k_{i}-n_{i}\right|-3}{2}\right]+1\right)^{m}} \chi_{\left\{\left|k_{i}-n_{i}\right| \geq 5\right\}} .
$$

Note that $m \geq 2$, then $\sum_{n_{i} \in \mathbb{Z}} \frac{1}{\left(2\left[\frac{\left|k_{i}-n_{i}\right|-3}{2}\right]+1\right)^{m}} \chi_{\left\{\left|k_{i}-n_{i}\right| \geq 5\right\}} \leq \sum_{\substack{n_{i} \in \mathbb{Z} \\\left|n_{i}\right| \geq 5}} \frac{1}{\left(2\left[\frac{\left|n_{i}\right|-3}{2}\right]+1\right)^{m}} \leq 4$.This together with (6.8) yields that

$$
\sum_{n_{i} \in \mathbb{Z}} \frac{1}{\left(F\left(r_{i}\left(n^{\prime}, n_{d}\right)\right)\right)^{m}} \chi_{\left\{\left|k_{i}-n_{i}\right|<2 r_{i}\left(n^{\prime}, n_{d}\right)\right\}} \leq 13 .
$$


Combining (6.9) with (6.7) gives that

$$
\sum_{n^{\prime} \in \mathbb{Z}^{d-1}} \sum_{n_{d} \in X_{n^{\prime}}^{+}} \prod_{i=1}^{d} \frac{1}{\left(F\left(r_{i}\left(n^{\prime}, n_{d}\right)\right)\right)^{m}} \sum_{\vec{k} \in R_{2 \vec{r}\left(n^{\prime}, n_{d}\right)}\left(n^{\prime}, n_{d}\right) \cap \mathbb{Z}^{d}}\left|D_{d} f_{\mu}(\vec{k})\right| \lesssim_{d}\left\|D_{d} f_{\mu}\right\|_{\ell^{1}\left(\mathbb{Z}^{d}\right)} .
$$

Then (6.4) follows immediately from (6.6) and (6.10).

6.2. The continuity part. Let $g_{i, j} \rightarrow f_{j}$ in $\ell^{1}\left(\mathbb{Z}^{d}\right)$ for all $1 \leq j \leq m$ when $i \rightarrow \infty$. For convenience, we set $\vec{g}_{i}=\left(g_{i, 1}, \ldots, g_{i, m}\right)$. It suffices to show that

$$
\lim _{i \rightarrow \infty}\left\|D_{l} \mathbb{M}_{\mathcal{R}}\left(\vec{g}_{i}\right)-D_{l} \mathbb{M}_{\mathcal{R}}(\vec{f})\right\|_{\ell^{1}\left(\mathbb{Z}^{d}\right)}=0, \quad \text { for each } 1 \leq l \leq d .
$$

We only prove (6.11) for the case $l=d$ and the other cases are similar. Since we have ||$D_{\ell}\left|g_{i, j}\right|-$ $D_{\ell}\left|f_{j}\right|\left\|_{\ell^{1}\left(\mathbb{Z}^{d}\right)} \leq 2\right\||| g_{i, j}|-| f_{j} \mid\left\|_{\ell^{1}\left(\mathbb{Z}^{d}\right)} \leq 2\right\| g_{i, j}-f_{j} \|_{\ell^{1}\left(\mathbb{Z}^{d}\right)}$, we may assume without loss of generality that all $g_{i, j} \geq 0$ and $f_{j} \geq 0$. Given $\epsilon \in(0,1)$, there exists $N_{1}=N_{1}(\epsilon, \vec{f}) \in \mathbb{N}$ such that for all $1 \leq j \leq m$ and $i \geq N_{1}$,

$$
\left\|g_{i, j}-f_{j}\right\|_{\ell^{1}\left(\mathbb{Z}^{d}\right)}<\epsilon \text { and }\left\|g_{i, j}\right\|_{\ell^{1}\left(\mathbb{Z}^{d}\right)} \leq\left\|f_{j}\right\|_{\ell^{1}\left(\mathbb{Z}^{d}\right)}+1 .
$$

For all $1 \leq j \leq m$ and $i \geq N_{1}$, it then follows that

$$
\left\|D_{d} g_{i, j}\right\|_{\ell^{1}\left(\mathbb{Z}^{d}\right)} \leq 2\left\|g_{i, j}\right\|_{\ell^{1}\left(\mathbb{Z}^{d}\right)} \leq 2\left(\left\|f_{j}\right\|_{\ell^{1}\left(\mathbb{Z}^{d}\right)}+1\right)
$$

On the other hand, by the boundedness part in Theorem 1.7 we obtain $D_{d} \mathbb{M}_{\mathcal{R}}(\vec{f}) \in \ell^{1}\left(\mathbb{Z}^{d}\right)$. Hence, for the above $\epsilon>0$, there exists $\Lambda_{1}>0$ such that

$$
\max \left\{\left\|D_{d} \mathbb{M}_{\mathcal{R}}(\vec{f}) \chi_{\left(R_{\overrightarrow{\Lambda_{1}}}(\overrightarrow{0})\right)^{c}}\right\|_{\ell^{1}\left(\mathbb{Z}^{d}\right)}, \sup _{1 \leq j \leq m}\left\|f_{j} \chi_{\left(R_{\overrightarrow{\Lambda_{1}}}(\overrightarrow{0})\right)^{c}}\right\|_{\ell^{1}\left(\mathbb{Z}^{d}\right)}\right\}<\epsilon .
$$

Here $\overrightarrow{\Lambda_{1}}=\left(\Lambda_{1}, \ldots, \Lambda_{1}\right)$. Since $m>1$, there exists an integer $\Lambda_{2}>0$ such that

$$
\Lambda_{2}^{1-m}<\epsilon .
$$

Step1: Reduction. When $i \geq N_{1}$, we get from (6.13) that

$$
\begin{aligned}
& \left|\mathbb{M}_{\mathcal{R}}\left(\vec{g}_{i}\right)(\vec{n})-\mathbb{M}_{\mathcal{R}}(\vec{f})(\vec{n})\right| \\
& \leq \sup _{\vec{n} \in R \in \mathcal{R}} \frac{1}{N(R)^{m}}\left|\prod_{i=1}^{m} \sum_{\vec{k} \in R \cap \mathbb{Z}^{d}} g_{i, j}(\vec{k})-\prod_{i=1}^{m} \sum_{\vec{k} \in R \cap \mathbb{Z}^{d}} f_{j}(\vec{k})\right| \\
& \leq \sum_{\mu=1}^{m} \sum_{\vec{k} \in R \cap \mathbb{Z}^{d}}\left|g_{i, \mu}(\vec{k})-f_{\mu}(\vec{k})\right|\left(\prod_{\nu=1}^{\mu-1} \sum_{\vec{k} \in R \cap \mathbb{Z}^{d}} g_{i, \iota}(\vec{k})\right)\left(\prod_{\nu=\mu+1}^{m} \sum_{\vec{k} \in R \cap \mathbb{Z}^{d}} f_{\nu}(\vec{k})\right) \\
& \leq \sum_{\mu=1}^{m}\left\|g_{i, \mu}-f_{\mu}\right\|_{\ell^{1}\left(\mathbb{Z}^{d}\right)}\left(\prod_{\nu \neq \mu, 1 \leq \nu \leq m}\left(\left\|f_{\nu}\right\|_{\ell^{1}\left(\mathbb{Z}^{d}\right)}+1\right)\right) .
\end{aligned}
$$

This implies that $\mathbb{M}_{\mathcal{R}}\left(\vec{g}_{i}\right)(\vec{n}) \rightarrow \mathbb{M}_{\mathcal{R}}(\vec{f})(\vec{n})$ as $i \rightarrow \infty$ for any $\vec{n} \in \mathbb{Z}^{d}$, and

$$
D_{d} \mathbb{M}_{\mathcal{R}}\left(\vec{g}_{i}\right)(\vec{n}) \rightarrow D_{d} \mathbb{M}_{\mathcal{R}}(\vec{f})(\vec{n}) \quad \text { as } i \rightarrow \infty, \quad \forall \vec{n} \in \mathbb{Z}^{d} .
$$

Let $\Lambda=\max \left\{\Lambda_{1}, \Lambda_{2}, 6\right\}$. It follows from (6.16) that there exists $N_{2}=N_{2}(\epsilon, \Lambda) \in \mathbb{N}$ such that

$$
\left|D_{d} \mathbb{M}_{\mathcal{R}}\left(\vec{g}_{i}\right)(\vec{n})-D_{d} \mathbb{M}_{\mathcal{R}}(\vec{f})(\vec{n})\right| \leq \frac{\epsilon}{\left(N\left(R_{\overrightarrow{2}}(\overrightarrow{0})\right)\right)}, \quad \forall i \geq N_{2} \text { and } \vec{n} \in R_{\vec{\Lambda}}(\overrightarrow{0}) \cap \mathbb{Z}^{d}
$$


Then, by (6.14) and (6.17), we have that for all $i \geq N_{2}$,

$$
\begin{aligned}
& \left\|D_{d} \mathbb{M}_{\mathcal{R}}\left(\vec{g}_{i}\right)-D_{d} \mathbb{M}_{\mathcal{R}}(\vec{f})\right\|_{\ell^{1}\left(\mathbb{Z}^{d}\right)}=\left\|\left(D_{d} \mathbb{M}_{\mathcal{R}}\left(\vec{g}_{i}\right)-D_{d} \mathbb{M}_{\mathcal{R}}(\vec{f})\right) \chi_{N\left(R_{2 \vec{\Lambda}}(\overrightarrow{0})\right)}\right\|_{\ell^{1}\left(\mathbb{Z}^{d}\right)} \\
& +\left\|\left(D_{d} \mathbb{M}_{\mathcal{R}}\left(\vec{g}_{i}\right)-D_{d} \mathbb{M}_{\mathcal{R}}(\vec{f})\right) \chi_{\left(N\left(R_{2 \vec{\Lambda}}(\overrightarrow{0})\right)\right)^{c}}\right\|_{\ell^{1}\left(\mathbb{Z}^{d}\right)} \\
& \leq 2 \epsilon+\left\|D_{d} \mathbb{M}_{\mathcal{R}}\left(\vec{g}_{i}\right) \chi_{\left(N\left(R_{2 \vec{\Lambda}}(\overrightarrow{0})\right)\right)^{c}}\right\|_{\ell^{1}\left(\mathbb{Z}^{d}\right)}
\end{aligned}
$$

Thus, to prove (6.11) for $l=d$, it suffices to show that

$$
\left\|D_{d} \mathbb{M}_{\mathcal{R}}\left(\vec{g}_{i}\right) \chi_{\left(N\left(R_{2 \vec{\Lambda}}(\overrightarrow{0})\right)\right)^{c}}\right\|_{\ell^{1}\left(\mathbb{Z}^{d}\right)} \lesssim_{d, m, \vec{f}} \epsilon, \quad \forall i \geq N_{1} .
$$

Step 2: Proof of (6.18). Note that

$$
\left(R_{2 \vec{\Lambda}}(\overrightarrow{0})\right)^{c} \cap \mathbb{Z}^{d} \subset \bigcup_{\mu=1}^{d} E_{\mu}:=\bigcup_{\mu=1}^{d} \mathbb{Z}^{d} \backslash\left\{\vec{n}=\left(n_{1}, \ldots, n_{d}\right) \in \mathbb{Z}^{d}:\left|n_{\mu}\right| \leq 2 \Lambda\right\} .
$$

Fix $j \geq N_{1}$. Then we have

$$
\left\|D_{d} \mathbb{M}_{\mathcal{R}}\left(\vec{g}_{i}\right) \chi_{\left(N\left(R_{2 \vec{\Lambda}}(\overrightarrow{0})\right)\right)^{c}}\right\|_{\ell^{1}\left(\mathbb{Z}^{d}\right)} \leq \sum_{\mu=1}^{d} A_{2, \mu}:=\sum_{\mu=1}^{d} \sum_{\vec{n} \in E_{\mu}}\left|D_{d} \mathbb{M}_{\mathcal{R}}\left(\vec{g}_{i}\right)(\vec{n})\right| .
$$

Step 3: Estimates for $A_{2, d}$. For each $n^{\prime} \in \mathbb{Z}^{d-1}$, let

$$
\begin{gathered}
Y_{n^{\prime}}^{+}=\left\{\left|n_{d}\right| \geq 2 \Lambda: \mathbb{M}_{\mathcal{R}}\left(\vec{g}_{i}\right)\left(n^{\prime}, n_{d}+1\right) \leq \mathbb{M}_{\mathcal{R}}\left(\vec{g}_{i}\right)\left(n^{\prime}, n_{d}\right)\right\}, \quad \text { and } \\
Y_{n^{\prime}}^{-}=\left\{\left|n_{d}\right| \geq 2 \Lambda: \mathbb{M}_{\mathcal{R}}\left(\vec{g}_{i}\right)\left(n^{\prime}, n_{d}+1\right)>\mathbb{M}_{\mathcal{R}}\left(\vec{g}_{i}\right)\left(n^{\prime}, n_{d}\right)\right\} .
\end{gathered}
$$

Then, we have

$$
\begin{aligned}
A_{2, d} \leq & \sum_{n^{\prime} \in \mathbb{Z}^{d-1}} \sum_{n_{d} \in Y_{n^{\prime}}^{+}}\left(\mathbb{M}_{\mathcal{R}}\left(\vec{g}_{i}\right)\left(n^{\prime}, n_{d}\right)-\mathbb{M}_{\mathcal{R}}\left(\vec{g}_{i}\right)\left(n^{\prime}, n_{d}+1\right)\right) \\
& +\sum_{n^{\prime} \in \mathbb{Z}^{d-1}} \sum_{n_{d} \in Y_{n^{\prime}}^{-}}\left(\mathbb{M}_{\mathcal{R}}\left(\vec{g}_{i}\right)\left(n^{\prime}, n_{d}+1\right)-\mathbb{M}_{\mathcal{R}}\left(\vec{g}_{i}\right)\left(n^{\prime}, n_{d}\right)\right)
\end{aligned}
$$

We want to show that

$$
\begin{aligned}
& \sum_{n^{\prime} \in \mathbb{Z}^{d-1}} \sum_{n_{d} \in Y_{n^{\prime}}^{+}}\left(\mathbb{M}_{\mathcal{R}}\left(\vec{g}_{i}\right)\left(n^{\prime}, n_{d}\right)-\mathbb{M}_{\mathcal{R}}\left(\vec{g}_{i}\right)\left(n^{\prime}, n_{d}+1\right)\right) \lesssim_{d, m, \vec{f}} \epsilon, \quad \forall i \geq N_{1} ; \\
& \sum_{n^{\prime} \in \mathbb{Z}^{d-1}} \sum_{n_{d} \in Y_{n^{\prime}}^{-}}\left(\mathbb{M}_{\mathcal{R}}\left(\vec{g}_{i}\right)\left(n^{\prime}, n_{d}+1\right)-\mathbb{M}_{\mathcal{R}}\left(\vec{g}_{i}\right)\left(n^{\prime}, n_{d}\right)\right) \lesssim_{d, m, \vec{f}} \epsilon, \quad \forall i \geq N_{1} .
\end{aligned}
$$

We will only prove (6.21), since (6.22) is analogous. Fix $i \geq N_{1}$. Since all $g_{i, j} \in \ell^{1}\left(\mathbb{Z}^{d}\right)$, then for any $\left(n^{\prime}, n_{d}\right) \in \mathbb{Z}^{d}$, there exist $\vec{x} \in \mathbb{R}^{d}$ and $\vec{r}\left(n^{\prime}, n_{d}\right) \in \mathbb{R}_{+}^{d}$ such that $\left(n^{\prime}, n_{d}\right) \in R_{\vec{r}\left(n^{\prime}, n_{d}\right)}(\vec{x})$ and $\mathbb{M}_{\mathcal{R}}\left(\overrightarrow{g_{i}}\right)\left(n^{\prime}, n_{d}\right)=A_{\vec{r}\left(n^{\prime}, n_{d}\right)}\left(\overrightarrow{g_{i}}\right)(\vec{x})$. Let $\vec{r}\left(n^{\prime}, n_{d}\right)=\left(r_{1}\left(n^{\prime}, n_{d}\right), \ldots, r_{d}\left(n^{\prime}, n_{d}\right)\right)$. By the similar arguments as in getting (6.6) and (6.7), we obtain

$$
\begin{aligned}
& \sum_{n^{\prime} \in \mathbb{Z}^{d-1}} \sum_{n_{d} \in Y_{n^{\prime}}^{+}}\left(\mathbb{M}_{\mathcal{R}}\left(\vec{g}_{i}\right)\left(n^{\prime}, n_{d}\right)-\mathbb{M}_{\mathcal{R}}\left(\vec{g}_{i}\right)\left(n^{\prime}, n_{d}+1\right)\right) \\
\leq & \sum_{\mu=1}^{m} \prod_{\nu \neq \mu, 1 \leq \nu \leq m}\left\|g_{i, \nu}\right\|_{\ell^{1}\left(\mathbb{Z}^{d}\right)} \\
& \times\left(\sum_{n^{\prime} \in \mathbb{Z}^{d-1}} \sum_{n_{d} \in Y_{n^{\prime}}^{+}} \prod_{i=1}^{d} \frac{1}{\left(F\left(r_{i}\left(n^{\prime}, n_{d}\right)\right)\right)^{m}} \sum_{\vec{k} \in R_{2 \vec{r}\left(n^{\prime}, n_{d}\right)}\left(n^{\prime}, n_{d}\right) \cap \mathbb{Z}^{d}}\left|D_{d} g_{i, \mu}(\vec{k})\right|\right)
\end{aligned}
$$




$$
\begin{aligned}
\leq & \sum_{\mu=1}^{m} \prod_{\nu \neq \mu, 1 \leq \nu \leq m}\left\|g_{i, \nu}\right\|_{\ell^{1}\left(\mathbb{Z}^{d}\right)} \\
& \times \sum_{\left(k_{1}, \ldots, k_{d}\right) \in \mathbb{Z}^{d}}\left|D_{d} g_{i, \mu}\left(k_{1}, \ldots, k_{d}\right)\right| \prod_{i=1}^{d}\left(\sum_{n_{i} \in \mathbb{Z}} \frac{1}{\left(F\left(r_{i}\left(n^{\prime}, n_{d}\right)\right)^{m}\right.} \chi_{\left\{\left|k_{i}-n_{i}\right|<2 r_{i}\left(n^{\prime}, n_{d}\right)\right\}}\right) .
\end{aligned}
$$

When $\left|k_{d}\right|>\Lambda$, by (6.9), we get

$$
\sum_{\substack{n_{d} \in \mathbb{Z} \\\left|n_{d}\right| \geq 2 \Lambda}} \frac{1}{\left(F\left(r_{d}\left(n^{\prime}, n_{d}\right)\right)\right)^{m}} \chi_{\left\{\left|k_{d}-n_{d}\right|<2 r_{d}\left(n^{\prime}, n_{d}\right)\right\}} \leq 13 .
$$

When $\left|k_{d}\right| \leq \Lambda$, then $\left|k_{d}-n_{d}\right| \geq \Lambda \geq 6$ for $\left|n_{d}\right| \geq 2 \Lambda$. This together with (6.15) yields that

$$
\begin{aligned}
& \sum_{\substack{n_{d} \in \mathbb{Z} \\
\left|n_{d}\right| \geq 2 \Lambda}} \frac{1}{\left(F\left(r_{d}\left(n^{\prime}, n_{d}\right)\right)\right)^{m}} \chi_{\left\{\left|k_{d}-n_{d}\right|<2 r_{d}\left(n^{\prime}, n_{d}\right)\right\}} \\
& \leq \sum_{\substack{n_{d} \in \mathbb{Z} \\
\left|n_{d}\right| \geq 2 \Lambda}} \frac{1}{\left(2\left[\frac{\left|k_{d}-n_{d}\right|-3}{2}\right]+1\right)^{m}} \chi_{\left\{\left|k_{d}-n_{d}\right| \geq \Lambda\right\}} \\
& \lesssim_{d, m} \epsilon .
\end{aligned}
$$

By (6.12)- (6.14) and (6.24)- -6.25$)$, we obtain

$$
\begin{aligned}
& \sum_{\left(k_{1}, \ldots, k_{d}\right) \in \mathbb{Z}^{d}}\left|D_{d} g_{i, \mu}\left(k_{1}, \ldots, k_{d}\right)\right| \prod_{i=1}^{d}\left(\sum_{n_{i} \in \mathbb{Z}} \frac{1}{\left(F\left(r_{i}\left(n^{\prime}, n_{d}\right)\right)\right)^{m}} \chi_{\left\{\left|k_{i}-n_{i}\right|<2 r_{i}\left(n^{\prime}, n_{d}\right)\right\}}\right) \\
& \lesssim d, m \sum_{k^{\prime} \in \mathbb{Z}^{d-1}} \sum_{\substack{k_{d} \in \mathbb{Z} \\
\left|k_{d}\right|>\Lambda}}\left|D_{d} g_{i, \mu}\left(k^{\prime}, k_{d}\right)\right|+\sum_{k^{\prime} \in \mathbb{Z}^{d-1}} \sum_{\substack{k_{d} \in \mathbb{Z} \\
\left|k_{d}\right| \leq \Lambda}}\left|D_{d} g_{i, \mu}\left(k^{\prime}, k_{d}\right)\right| \epsilon \\
& \lesssim_{d, m}\left\|g_{i, \mu}-f_{\mu}\right\|_{\ell^{1}\left(\mathbb{Z}^{d}\right)}+\left\|f_{\mu} \chi_{\left.\left(R_{\vec{\Lambda}}(\overrightarrow{0})\right)^{c}\right)}\right\|_{\ell^{1}\left(\mathbb{Z}^{d}\right)}+2\left(\left\|f_{\mu}\right\|_{\ell^{1}\left(\mathbb{Z}^{d}\right)}+1\right) \epsilon \\
& \lesssim d, m, f_{\mu} \epsilon \text {. }
\end{aligned}
$$

(6.26) together with (6.12)-(6.13) and (6.23) yields (6.21). It follows from (6.20)-(6.22) that

$$
A_{2, d} \lesssim_{d, m, \vec{f}} \epsilon, \quad \forall i \geq N_{1} .
$$

Step 4: Estimates for $A_{2, \mu}$ with $\mu=1,2, \ldots, d-1$. We first estimates $A_{2,1}$. For each $n^{\prime} \in \mathbb{Z}^{d-1}$, let

$$
\begin{aligned}
& Z_{n^{\prime}}^{+}=\left\{n_{d} \in \mathbb{Z}: \mathbb{M}_{\mathcal{R}}\left(\vec{g}_{i}\right)\left(n^{\prime}, n_{d}+1\right) \leq \mathbb{M}_{\mathcal{R}}\left(\vec{g}_{i}\right)\left(n^{\prime}, n_{d}\right)\right\}, \\
& Z_{n^{\prime}}^{-}=\left\{n_{d} \in \mathbb{Z}: \mathbb{M}_{\mathcal{R}}\left(\vec{g}_{i}\right)\left(n^{\prime}, n_{d}+1\right)>\mathbb{M}_{\mathcal{R}}\left(\vec{g}_{i}\right)\left(n^{\prime}, n_{d}\right)\right\} .
\end{aligned}
$$

Then we have

$$
\begin{aligned}
A_{2,1}= & \sum_{\left|n_{1}\right|>2 \Lambda} \sum_{n^{\prime} \in \mathbb{Z}^{d-1}}\left|\mathbb{M}_{\mathcal{R}}\left(\vec{g}_{i}\right)\left(n_{1}, \ldots, n_{d-1}, n_{d}+1\right)-\mathbb{M}_{\mathcal{R}}\left(\vec{g}_{i}\right)\left(n_{1}, \ldots, n_{d}\right)\right| \\
= & \sum_{\substack{n^{\prime} \in \mathbb{Z}^{d-1} \\
\left|n_{1}\right|>2 \Lambda}} \sum_{n_{d} \in Z_{n^{\prime}}^{+}}\left(\mathbb{M}_{\mathcal{R}}\left(\vec{g}_{i}\right)\left(n^{\prime}, n_{d}\right)-\mathbb{M}_{\mathcal{R}}\left(\vec{g}_{i}\right)\left(n^{\prime}, n_{d}+1\right)\right) \\
& +\sum_{\substack{n^{\prime} \in \mathbb{Z}^{d-1} \\
\left|n_{1}\right|>2 \Lambda}} \sum_{n_{d} \in Z_{n^{\prime}}^{-}}\left(\mathbb{M}_{\mathcal{R}}\left(\vec{g}_{i}\right)\left(n^{\prime}, n_{d}+1\right)-\mathbb{M}_{\mathcal{R}}\left(\vec{g}_{i}\right)\left(n^{\prime}, n_{d}\right)\right) .
\end{aligned}
$$


We want to show that

$$
\begin{aligned}
& \sum_{\substack{n^{\prime} \in \mathbb{Z}^{d}-1 \\
\left|n_{1}\right|>2 \Lambda}} \sum_{n_{d} \in Z_{n^{\prime}}^{+}}\left(\mathbb{M}_{\mathcal{R}}\left(\vec{g}_{i}\right)\left(n^{\prime}, n_{d}\right)-\mathbb{M}_{\mathcal{R}}\left(\vec{g}_{i}\right)\left(n^{\prime}, n_{d}+1\right)\right) \lesssim_{d, m, \vec{f}} \epsilon, \quad \forall i \geq N_{1} ; \\
& \sum_{\substack{n^{\prime} \in \mathbb{Z}^{d}-1 \\
\left|n_{1}\right|>2 \Lambda}} \sum_{n_{d} \in Z_{n^{\prime}}^{-}}\left(\mathbb{M}_{\mathcal{R}}\left(\vec{g}_{i}\right)\left(n^{\prime}, n_{d}+1\right)-\mathbb{M}_{\mathcal{R}}\left(\vec{g}_{i}\right)\left(n^{\prime}, n_{d}\right)\right) \lesssim_{d, m, \vec{f}} \epsilon, \quad \forall i \geq N_{1} .
\end{aligned}
$$

We will only prove (6.29), since (6.30) is analogous. By the similar arguments as in getting (6.23), for any $\left(n^{\prime}, n_{d}\right) \in \mathbb{Z}^{d}$, there exists $\vec{r}\left(n^{\prime}, n_{d}\right)=\left(r_{1}\left(n^{\prime}, n_{d}\right), \ldots, r_{d}\left(n^{\prime}, n_{d}\right)\right) \in \mathbb{R}_{+}^{d}$ such that

$$
\begin{aligned}
& \sum_{\substack{n^{\prime} \in \mathbb{Z}^{d-1} \\
\left|n_{1}\right|>2 \Lambda}} \sum_{n_{d} \in Z_{n^{\prime}}^{+}}\left(\mathrm{M}_{\mathcal{R}} f_{j}\left(n^{\prime}, n_{d}\right)-\mathrm{M}_{\mathcal{R}} f_{j}\left(n^{\prime}, n_{d}+1\right)\right) \\
& \lesssim_{m} \sum_{\mu=1}^{m} \prod_{\nu \neq \mu, 1 \leq \nu \leq m}\left\|g_{i, \nu}\right\|_{\ell^{1}\left(\mathbb{Z}^{d}\right)} \\
& \quad \times \sum_{\substack{\left.k_{1}, \ldots, k_{d}\right) \in \mathbb{Z}^{d} \\
d}}\left|D_{d} g_{i, \mu}\left(k_{1}, \ldots, k_{d}\right)\right|\left(\sum_{\left|n_{1}\right|>2 \Lambda} \frac{1}{\left(F\left(r_{1}\left(n^{\prime}, n_{d}\right)\right)\right)^{m}} \chi_{\left\{\left|k_{1}-n_{1}\right|<2 r_{1}\left(n^{\prime}, n_{d}\right)\right\}}\right) \\
& \quad \times \prod_{i=2}^{d}\left(\sum_{n_{i} \in \mathbb{Z}} \frac{1}{\left(F\left(r_{i}\left(n^{\prime}, n_{d}\right)\right)\right)^{m}} \chi_{\left\{\left|k_{i}-n_{i}\right|<2 r_{i}\left(n^{\prime}, n_{d}\right)\right\}}\right) .
\end{aligned}
$$

When $\left|k_{1}\right| \leq \Lambda$, then $\left|k_{1}-n_{1}\right| \geq \Lambda \geq 6$ for $\left|n_{1}\right| \geq 2 \Lambda$. By the arguments similar to those used in deriving (6.25), we can obtain

$$
\sum_{\left|n_{1}\right|>2 \Lambda} \frac{1}{\left(F\left(r_{1}\left(n^{\prime}, n_{d}\right)\right)\right)^{m}} \chi_{\left\{\left|k_{1}-n_{1}\right|<2 r_{1}\left(n^{\prime}, n_{d}\right)\right\}} \lesssim_{d, m} \epsilon
$$

When $\left|k_{1}\right|>\Lambda$, by (6.9), we get

$$
\sum_{\substack{n_{d} \in \mathbb{Z} \\\left|n_{1}\right| \geq 2 \Lambda}} \frac{1}{\left(F\left(r_{1}\left(n^{\prime}, n_{d}\right)\right)\right)^{m}} \chi_{\left\{\left|k_{1}-n_{1}\right|<2 r_{1}\left(n^{\prime}, n_{d}\right)\right\}} \leq 13 .
$$

Then (6.29) follows from (6.31)-(6.33) and (6.13)-(6.14). By (6.28)-(6.30), it holds that

$$
A_{2,1} \lesssim_{d, m, \vec{f}} \epsilon, \quad \forall i \geq N_{1}
$$

Similarly, we can get

$$
A_{2, \mu} \lesssim_{d, m, \vec{f}} \epsilon, \quad \forall i \geq N_{1} \text { and } \mu=2, \ldots, d-1 .
$$

(6.35) together with (6.19), (6.27) and (6.34) yields (6.18). Thus, the proof of the continuity part is complete.

\section{Properties of $u_{x, \vec{f}}$}

We summrize the properties of $u_{x, \vec{f}}$ into nine Claims. The proofs of them are thoroughly elementary. In what follow, we set $\overline{\mathbb{Q}}_{+}=\mathbb{Q}_{+} \cup\{0\}$.

Claim 1. Let $1<p_{j}<\infty$ and $f_{j} \in L^{p_{j}}\left(\mathbb{R}^{2}\right)$ for $1 \leq j \leq m$. Then

$$
\lim _{\substack{\left(r_{1,1}, r_{1,2}, r_{2,1}, r_{2,2}\right) \in \mathbb{R}_{+}^{4} \\ r_{1,1}+r_{1,2}+r_{2,1}+r_{2,2} \rightarrow \infty}} u_{x, \vec{f}}\left(r_{1,1}, r_{1,2}, r_{2,1}, r_{2,2}\right)=0 \text { for a.e. } x \in \mathbb{R}^{2} \text {. }
$$


Proof. Fix $1 \leq j \leq m$. We note first

$$
\begin{gathered}
\left\|f_{j}\left(x_{1}, \cdot\right)\right\|_{L^{p_{j}(\mathbb{R})}},\left\|\widetilde{\mathcal{M}}_{1} f_{j}\left(x_{1}, \cdot\right)\right\|_{L^{p_{j}(\mathbb{R})}}<\infty \text { a.e } x_{1} \in \mathbb{R}, \quad \text { and } \\
\left\|f_{j}\left(\cdot, x_{2}\right)\right\|_{L^{p_{j}(\mathbb{R})}},\left\|\widetilde{\mathcal{M}}_{2} f_{j}\left(\cdot, x_{2}\right)\right\|_{L^{p_{j}(\mathbb{R})}}<\infty \text { a.e } x_{2} \in \mathbb{R} .
\end{gathered}
$$

Let $\vec{r}=\left(r_{1,1}, r_{1,2}, r_{2,1}, r_{2,2}\right) \in \overline{\mathbb{R}}_{+}^{4}$. We consider the following three cases.

(a) $r_{1,1}+r_{1,2}>0$ and $r_{2,1}+r_{2,2}>0$. By Hölder's inequality, one finds that

$$
u_{x, \vec{f}}(\vec{r}) \leq \prod_{j=1}^{m} \frac{1}{r_{1,1}+r_{1,2}} \int_{x_{1}-r_{1,1}}^{x_{1}+r_{1,2}} \widetilde{\mathcal{M}}_{2} f_{j}\left(y_{1}, x_{2}\right) d y_{1} \leq \prod_{j=1}^{m} \frac{1}{\left(r_{1,1}+r_{1,2}\right)^{1 / p_{j}}}\left\|\widetilde{\mathcal{M}}_{2} f_{j}\left(\cdot, x_{2}\right)\right\|_{L^{p_{j}(\mathbb{R})}} .
$$

Simiarly we get

$$
u_{x, \vec{f}}(\vec{r}) \leq \prod_{j=1}^{m} \frac{1}{\left(r_{2,1}+r_{2,2}\right)^{1 / p_{j}}}\left\|\widetilde{\mathcal{M}}_{1} f_{j}\left(x_{1} \cdot\right)\right\|_{L^{p_{j}(\mathbb{R})}}
$$

So, we have

$$
u_{x, \vec{f}}(\vec{r}) \leq \min \left\{\prod_{j=1}^{m} \frac{1}{\left(r_{1,1}+r_{1,2}\right)^{1 / p_{j}}}\left\|\widetilde{\mathcal{M}}_{2} f_{j}\left(\cdot, x_{2}\right)\right\|_{L^{p_{j}}(\mathbb{R})}, \prod_{j=1}^{m} \frac{1}{\left(r_{2,1}+r_{2,2}\right)^{1 / p_{j}}}\left\|\widetilde{\mathcal{M}}_{1} f_{j}\left(x_{1} \cdot\right)\right\|_{L^{p_{j}(\mathbb{R})}}\right\} .
$$

(b) $r_{1,1}=r_{1,2}=0$ and $r_{2,1}+r_{2,2}>0$. By Hölder's inequality,

$$
u_{x, \vec{f}}(\vec{r})=\prod_{j=1}^{m} \frac{1}{r_{2,1}+r_{2,2}} \int_{x_{2}-r_{2,1}}^{x_{2}+r_{2,2}} f_{j}\left(x_{1}, y_{2}\right) d y_{2} \leq \prod_{j=1}^{m} \frac{1}{\left(r_{2,1}+r_{2,2}\right)^{1 / p_{j}}}\left\|f\left(x_{1}, \cdot\right)\right\|_{L^{p_{j}(\mathbb{R})}} .
$$

(c) $r_{1,1}+r_{1,2}>0$ and $r_{2,1}=r_{2,2}=0$. Similarly to (b) we get

$$
u_{x, \vec{f}}(\vec{r}) \leq \prod_{j=1}^{m} \frac{1}{\left(r_{1,1}+r_{1,2}\right)^{1 / p_{j}}}\left\|f_{j}\left(\cdot, x_{2}\right)\right\|_{L^{p_{j}(\mathbb{R})}} .
$$

From (a), (b) and (c), we see that (7.1) holds for a.e. $x \in \mathbb{R}^{2}$.

Claim 2. Let $1 \leq j \leq m$ and $f_{j} \in L_{\mathrm{loc}}^{1}\left(\mathbb{R}^{2}\right)$. Then the set $A_{j}$

$$
A_{j}:=\left\{\left(x_{1}, x_{2}\right) \in \mathbb{R}^{2}: \lim _{\substack{\left(r_{1,1}, r_{1,2}\right) \mathbb{R}_{+}^{2} \\ r_{1,1}+r_{1,2} \rightarrow 0}} \frac{1}{r_{1,1}+r_{1,2}} \int_{x_{1}-r_{1,1}}^{x_{1}+r_{1,2}}\left|f_{j}\left(y_{1}, x_{2}\right)-f_{j}\left(x_{1}, x_{2}\right)\right| d y_{1}=0\right\}
$$

is a measurable set in $\mathbb{R}^{2}$.

Proof. $\left(x_{1}, x_{2}\right) \in A_{j}$ is equivalent to the following: For any $k \in \mathbb{N}$, there exists an $\ell \in \mathbb{N}$ such that for $r_{1,1}, r_{1,2} \geq 0$ with $r_{1,1}+r_{1,2}<1 / \ell$,

$$
\frac{1}{r_{1,1}+r_{1,2}} \int_{x_{1}-r_{1,1}}^{x_{1}+r_{1,2}}\left|f_{j}\left(y_{1}, x_{2}\right)-f_{j}\left(x_{1}, x_{2}\right)\right| d y_{1}<\frac{1}{k} .
$$

And this is equivalent to: For any $k \in \mathbb{N}$, there exists an $\ell \in \mathbb{N}$ such that for $r_{1,1}, r_{1,2} \in \mathbb{Q}_{+}$with $r_{1,1}+r_{1,2}<1 / \ell$,

$$
\frac{1}{r_{1,1}+r_{1,2}} \int_{x_{1}-r_{1,1}}^{x_{1}+r_{1,2}}\left|f_{j}\left(y_{1}, x_{2}\right)-f_{j}\left(x_{1}, x_{2}\right)\right| d y_{1}<\frac{1}{k} .
$$

Thus, $A_{j}$ can be written in the following form:

$$
A_{j}=\bigcap_{k \in \mathbb{N}} \bigcup_{\ell \in \mathbb{N}} \bigcap_{\substack{\left(r_{1,1}, r_{1,2}\right) \in \mathbb{Q}_{+}^{2} \\ r_{1,1}+r_{1,2}<1 / \ell}}\left\{\left(x_{1}, x_{2}\right) \in \mathbb{R}^{2}: \frac{1}{r_{1,1}+r_{1,2}} \int_{x_{1}-r_{1,1}}^{x_{1}+r_{1,2}}\left|f_{j}\left(y_{1}, x_{2}\right)-f_{j}\left(x_{1}, x_{2}\right)\right| d y_{1}<\frac{1}{k}\right\} .
$$


Since $f_{j} \in L_{\text {loc }}^{1}\left(\mathbb{R}^{2}\right)$, we see that the function $\frac{1}{r_{1,1}+r_{1,2}} \int_{x_{1}-r_{1,1}}^{x_{1}+r_{1,2}}\left|f_{j}\left(y_{1}, x_{2}\right)-f_{j}\left(x_{1}, x_{2}\right)\right| d y_{1}$ is measurable in $\mathbb{R}^{2}$, and hence

$$
\left\{\left(x_{1}, x_{2}\right) \in \mathbb{R}^{2}: \frac{1}{r_{1,1}+r_{1,2}} \int_{x_{1}-r_{1,1}}^{x_{1}+r_{1,2}}\left|f_{j}\left(y_{1}, x_{2}\right)-f_{j}\left(x_{1}, x_{2}\right)\right| d y_{1}<\frac{1}{k}\right\}
$$

is a measurable set in $\mathbb{R}^{2}$. Thus, $A_{j}$ is a measurable set in $\mathbb{R}^{2}$.

Claim 3. Let $1 \leq j \leq m, f_{j} \in L_{\mathrm{loc}}^{1}\left(\mathbb{R}^{2}\right)$ and $r_{1,1}+r_{1,2}>0$. Then the set $B_{j}$

$$
\begin{aligned}
B_{j}:=\left\{\left(x_{1}, x_{2}\right) \in \mathbb{R}^{2}: \lim _{\substack{\left(r_{2,1}, r_{2,2}\right) \in \overline{\mathbb{R}}_{+}^{2} \\
r_{2,1}+r_{2,2} \rightarrow 0}} \frac{1}{r_{2,1}+r_{2,2}}\right. & \int_{x_{2}-r_{2,1}}^{x_{2}+r_{2,2}} \mid \frac{1}{r_{1,1}+r_{1,2}} \int_{x_{1}-r_{1,1}}^{x_{1}+r_{1,2}} f_{j}\left(y_{1}, y_{2}\right) d y_{1} \\
& \left.-\frac{1}{r_{1,1}+r_{1,2}} \int_{x_{1}-r_{1,1}}^{x_{1}+r_{1,2}} f_{j}\left(y_{1}, x_{2}\right) d y_{1} \mid d y_{2}=0\right\}
\end{aligned}
$$

is a measurable set in $\mathbb{R}^{2}$.

Proof. As in the proof of Claim 2, we can write

$$
\begin{aligned}
B_{j}=\bigcap_{k \in \mathbb{N}} \bigcup_{\substack { \ell \in \mathbb{N} \\
\begin{subarray}{c}{\left(r_{2,1}, r_{2,2}\right) \in \mathbb{Q}_{+}^{2} \\
r_{2,1}+r_{2,2}<1 / \ell{ \ell \in \mathbb { N } \\
\begin{subarray} { c } { ( r _ { 2 , 1 } , r _ { 2 , 2 } ) \in \mathbb { Q } _ { + } ^ { 2 } \\
r _ { 2 , 1 } + r _ { 2 , 2 } < 1 / \ell } }\end{subarray}}\left\{\left(x_{1}, x_{2}\right) \in \mathbb{R}^{2}\right. & : \frac{1}{r_{2,1}+r_{2,2}} \int_{x_{2}-r_{2,1}}^{x_{2}+r_{2,2}} \mid \frac{1}{r_{1,1}+r_{1,2}} \int_{x_{1}-r_{1,1}}^{x_{1}+r_{1,2}} f_{j}\left(y_{1}, y_{2}\right) d y_{1} \\
& \left.-\frac{1}{r_{1,1}+r_{1,2}} \int_{x_{1}-r_{1,1}}^{x_{1}+r_{1,2}} f_{j}\left(y_{1}, x_{2}\right) d y_{1} \mid d y_{2}<\frac{1}{k}\right\} .
\end{aligned}
$$

Hence as in the proof of Claim 2 , we see that $B_{j}$ is a measurable set in $\mathbb{R}^{2}$.

Claim 4. Let $1<p_{j}<\infty$ and $f_{j} \in L^{p_{j}}\left(\mathbb{R}^{2}\right)$ for $1 \leq j \leq m$. Then



Proof. To prove (7.2), it suffices to show that for fixed $1 \leq j \leq m$, there exists a null set $E_{1}$ in $\mathbb{R}^{2}$ such that for $\left(x_{1}, x_{2}\right) \in \mathbb{R}^{2} \backslash E_{1}$,

$$
\lim _{\substack{\left(r_{1,1}, r_{1,2}\right) \in \mathbb{R}_{+}^{2} \\ r_{1,1}+r_{1,2} \rightarrow 0}} \frac{1}{r_{1,1}+r_{1,2}} \int_{x_{1}-r_{1,1}}^{x_{1}+r_{1,2}}\left|f_{j}\left(y_{1}, x_{2}\right)-f_{j}\left(x_{1}, x_{2}\right)\right| d y_{1}=0 .
$$

From $f_{j} \in L^{p_{j}}\left(\mathbb{R}^{2}\right)$ it follows that $f_{j}\left(\cdot, x_{2}\right) \in L^{p_{j}}(\mathbb{R})$ a.e $x_{2} \in \mathbb{R}$. Hence for these $x_{2}$, by Lebesgue's differentiation theorem (note $p_{j}>1$ ) we see that (7.3) holds for a.e. $x_{1} \in \mathbb{R}$. By Claim 2, we see that there exists a null set $E_{1}$ in $\mathbb{R}^{2}$ such that (7.3) holds for $x \in \mathbb{R}^{2} \backslash E_{1}$.

Applying the arguments similar to those used in deriving Claim 4, we can get the following claim. The details are omitted.

Claim 5. Let $1<p_{j}<\infty$ and $f_{j} \in L^{p_{j}}\left(\mathbb{R}^{2}\right)$ for $1 \leq j \leq m$. Then

$$
\lim _{\substack{\left(r_{2,1}, r_{2,2}\right) \in \overline{\mathbb{R}}_{+}^{2} \\ r_{2,1}+r_{2,2} \rightarrow 0}} u_{x, \vec{f}^{-}}\left(0,0, r_{2,1}, r_{2,2}\right)=u_{x, \vec{f}^{-}}(0,0,0,0) \text { for a.e. } x \in \mathbb{R}^{2} .
$$


Claim 6. Let $1<p_{j}<\infty$ and $f_{j} \in L^{p_{j}}\left(\mathbb{R}^{2}\right)$ for $1 \leq j \leq m$. Then there exists a null set $E_{\mathbb{Q}}$ in $\mathbb{R}^{2}$ such that for $\left(x_{1}, x_{2}\right) \in \mathbb{R}^{2} \backslash E_{\mathbb{Q}}$ and $\left(r_{1,1}, r_{1,2}\right) \in \overline{\mathbb{Q}}_{+}^{2}$ with $r_{1,1}+r_{1,2}>0$,

$$
\begin{gathered}
\lim _{\substack{\left(r_{2,1}, r_{2,2}\right) \in \overline{\mathbb{R}}_{+}^{2} \\
r_{2,1}+r_{2,2} \rightarrow 0}} \frac{1}{r_{2,1}+r_{2,2}} \int_{x_{2}-r_{2,1}}^{x_{2}+r_{2,2}} \mid \frac{1}{r_{1,1}+r_{1,2}} \int_{x_{1}-r_{1,1}}^{x_{1}+r_{1,2}} f_{j}\left(y_{1}, y_{2}\right) d y_{1} \\
-\frac{1}{r_{1,1}+r_{1,2}} \int_{x_{1}-r_{1,1}}^{x_{1}+r_{1,2}} f_{j}\left(y_{1}, x_{2}\right) d y_{1} \mid d y_{2}=0 .
\end{gathered}
$$

Proof. Let $\left(r_{1,1}, r_{1,2}\right) \in \overline{\mathbb{R}}_{+}^{2}$ with $r_{1,1}+r_{1,2}>0$. From $f_{j} \in L^{p_{j}}\left(\mathbb{R}^{2}\right)$ we see that for all $x_{1} \in \mathbb{R}$,

$$
\left|\frac{1}{r_{1,1}+r_{1,2}} \int_{x_{1}-r_{1,1}}^{x_{1}+r_{1,2}} f_{j}\left(y_{1}, y_{2}\right) d y_{1}\right| \leq \frac{1}{\left(r_{1,1}+r_{1,2}\right)^{1 / p}}\left\|f_{j}\left(\cdot, y_{2}\right)\right\|_{L^{p_{j}(\mathbb{R})}} .
$$

and hence

$$
\left(\int_{\mathbb{R}}\left|\frac{1}{r_{1,1}+r_{1,2}} \int_{x_{1}-r_{1,1}}^{x_{1}+r_{1,2}} f_{j}\left(y_{1}, y_{2}\right) d y_{1}\right|^{p} d y_{2}\right)^{1 / p} \leq \frac{1}{\left(r_{1,1}+r_{1,2}\right)^{1 / p}}\left\|f_{j}\right\|_{L^{p_{j}\left(\mathbb{R}^{2}\right)}} .
$$

Hence, for every $x_{1} \in \mathbb{R}$, by Lebesgue's differentiation theorem, (17.4) holds for a.e. $x_{2} \in \mathbb{R}$. So, by Claim 2, there exists a null set $E_{r_{1,1}, r_{1,2}} \in \mathbb{R}^{2}$ such that (7.4) holds for $\left(x_{1}, x_{2}\right) \in \mathbb{R}^{2} \backslash E_{r_{1,1}, r_{1,2}}$. Now set

$$
E_{\mathbb{Q}}=\bigcup_{\substack{r_{1,1}, r_{1,2} \in \overline{\mathbb{Q}}_{+} \\ r_{1,1}+r_{1,2}>0}} E_{r_{1,1}, r_{1,2}}
$$

Then $\left|E_{\mathbb{Q}}\right|=0$ and for $\left(x_{1}, x_{2}\right) \in \mathbb{R}^{2} \backslash E_{\mathbb{Q}}$, (7.4) holds for $\left(r_{1,1}, r_{1,2}\right) \in \overline{\mathbb{Q}}_{+}^{2}$ with $r_{1,1}+r_{1,2}>0$.

Claim 7. Let $1<p_{j}<\infty$ and $f_{j} \in L^{p_{j}}\left(\mathbb{R}^{2}\right)$ for $1 \leq j \leq m$. Then there exists a null set $E_{2}$ in $\mathbb{R}^{2}$ such that for $\left(x_{1}, x_{2}\right) \in \mathbb{R}^{2} \backslash E_{2}$ and $\left(r_{1,1}, r_{1,2}\right) \in \overline{\mathbb{R}}_{+}^{2}$ with $r_{1,1}+r_{1,2}>0$,

$$
\lim _{\substack{\left.\left(r_{1,1}^{\prime}, r_{1,2}^{\prime}\right) \in \overline{\mathbb{R}}_{+}^{2} \\ r_{1,1}^{\prime}, r_{1,2}^{\prime}\right) \rightarrow\left(r_{1,1}, r_{1,2}\right)}} \frac{1}{r_{1,1}^{\prime}+r_{1,2}^{\prime}} \int_{x_{1}-r_{1,1}^{\prime}}^{x_{1}+r_{1,2}^{\prime}} f_{j}\left(y_{1}, x_{2}\right) d y_{1}=\frac{1}{r_{1,1}+r_{1,2}} \int_{x_{1}-r_{1,1}}^{x_{1}+r_{1,2}} f_{j}\left(y_{1}, x_{2}\right) d y_{1},
$$

for $1 \leq j \leq m$,

Proof. Fix $\left(r_{1,1}^{\prime}, r_{1,2}^{\prime}\right) \in \overline{\mathbb{R}}_{+}^{2}$, we have

$$
\begin{aligned}
& \left|\frac{1}{r_{1,1}^{\prime}+r_{1,2}^{\prime}} \int_{x_{1}-r_{1,1}^{\prime}}^{x_{1}+r_{1,2}^{\prime}} f_{j}\left(y_{1}, x_{2}\right) d y_{1}-\frac{1}{r_{1,1}+r_{1,2}} \int_{x_{1}-r_{1,1}}^{x_{1}+r_{1,2}} f_{j}\left(y_{1}, x_{2}\right) d y_{1}\right| \\
& \leq\left|\frac{1}{r_{1,1}^{\prime}+r_{1,2}^{\prime}}-\frac{1}{r_{1,1}+r_{1,2}}\right| \int_{x_{1}-r_{1,1}^{\prime}}^{x_{1}+r_{1,2}^{\prime}}\left|f_{j}\left(y_{1}, x_{2}\right)\right| d y_{1} \\
& \quad+\frac{1}{r_{1,1}+r_{1,2}}\left(\int_{x_{1}+\min \left(r_{1,2}^{\prime}, r_{1,2}\right)}^{x_{1}+\max \left(r_{1,2}^{\prime}, r_{1,2}\right)}\left|f_{j}\left(y_{1}, x_{2}\right)\right| d y_{1}+\int_{x_{1}-\max \left(r_{1,1}^{\prime}, r_{1,1}\right)}^{x_{1}-\min \left(r_{1,1}^{\prime}, r_{1,1}\right)}\left|f_{j}\left(y_{1}, x_{2}\right)\right| d y_{1}\right) \\
& \leq \\
& \quad \frac{\left|r_{1,1}-r_{1,1}^{\prime}\right|+\left|r_{1,2}-r_{1,2}^{\prime}\right|}{\left(r_{1,1}+r_{1,2}\right)\left(r_{1,1}^{\prime}+r_{1,2}^{\prime}\right)}\left(r_{1,1}^{\prime}+r_{1,2}^{\prime}\right)^{1 / p_{j}^{\prime}}\left\|f_{j}\left(\cdot, x_{2}\right)\right\|_{L^{p_{j}}(\mathbb{R})} \\
& \quad+\frac{\left|r_{1,1}-r_{1,1}^{\prime}\right|^{1 / p_{j}^{\prime}}+\left|r_{1,2}-r_{1,2}^{\prime}\right|^{1 / p_{j}^{\prime}}}{r_{1,1}+r_{1,2}}\left\|f_{j}\left(\cdot, x_{2}\right)\right\|_{L^{p_{j}}(\mathbb{R})} .
\end{aligned}
$$


Now, there exists a null set $E_{2,1}$ in $\mathbb{R}$ such that $\left\|f_{j}\left(\cdot, x_{2}\right)\right\|_{L^{p_{j}(\mathbb{R})}}<\infty$ for $x_{2} \in \mathbb{R} \backslash E_{2,1}$. Set $E_{2}=\mathbb{R} \times E_{2,1}$. Then $E_{2}$ ia a null set in $\mathbb{R}^{2}$. And for $\left(x_{1}, x_{2}\right) \in \mathbb{R}^{2} \backslash E_{2}$, (7.5) holds.

Claim 8. Let $1<p_{j}<\infty$ and $f_{j} \in L^{p_{j}}\left(\mathbb{R}^{2}\right)$ for $1 \leq j \leq m$. Then there exists a null set $E_{3}$ in $\mathbb{R}^{2}$ such that for $\left(x_{1}, x_{2}\right) \in \mathbb{R}^{2} \backslash E_{3}$ and $\left(r_{1,1}, r_{1,2}, r_{2,1}, r_{2,2}\right) \in \overline{\mathbb{R}}_{+}^{4}$ with $r_{1,1}+r_{1,2}>0$ and $r_{2,1}+r_{2,2}>0$,

$$
\begin{gathered}
\lim _{\substack{\left(r_{1,1}^{\prime}, r_{1,2}^{\prime}\right) \in \overline{\mathbb{R}}_{+}^{2} \\
\left(r_{1,1}^{\prime}, r_{1,2}^{\prime}\right) \rightarrow\left(r_{1,1}, r_{1,2}\right)}} \frac{1}{r_{2,1}+r_{2,2}} \int_{x_{2}-r_{2,1}}^{x_{2}+r_{2,2}} \mid \frac{1}{r_{1,1}+r_{1,2}} \int_{x_{1}-r_{1,1}}^{x_{1}+r_{1,2}} f_{j}\left(y_{1}, y_{2}\right) d y_{1} \\
-\frac{1}{r_{1,1}^{\prime}+r_{1,2}^{\prime}} \int_{x_{1}-r_{1,1}^{\prime}}^{x_{1}+r_{1,2}^{\prime}} f_{j}\left(y_{1}, y_{2}\right) d y_{1} \mid d y_{2}=0
\end{gathered}
$$

Proof.

The left side of (7.6)

$$
\begin{aligned}
& \leq \frac{1}{r_{2,1}+r_{2,2}} \int_{x_{2}-r_{2,1}}^{x_{2}+r_{2,2}}\left(\left|\frac{1}{r_{1,1}+r_{1,2}}-\frac{1}{r_{1,1}^{\prime}+r_{1,2}^{\prime}}\right| \int_{x_{1}-r_{1,1}}^{x_{1}+r_{1,2}}\left|f_{j}\left(y_{1}, y_{2}\right)\right| d y_{1}\right. \\
& \left.\quad+\frac{1}{r_{1,1}^{\prime}+r_{1,2}^{\prime}}\left(\int_{x_{1}+\min \left(r_{1,2}^{\prime}, r_{1,2}\right)}^{x_{1}+\max \left(r_{1,2}^{\prime}, r_{1,2}\right)}\left|f_{j}\left(y_{1}, y_{2}\right)\right| d y_{1}+\int_{x_{1}-\max \left(r_{1,1}^{\prime}, r_{1,1}\right)}^{x_{1}-\min \left(r_{1,1}^{\prime}, r_{1,1}\right)}\left|f_{j}\left(y_{1}, y_{2}\right)\right| d y_{1}\right)\right) d y_{2} \\
& \leq \frac{\left|r_{1,1}-r_{1,1}^{\prime}\right|+\left|r_{1,2}-r_{1,2}^{\prime}\right|}{\left(r_{1,1}+r_{1,2}\right)^{2}\left(r_{1,1}^{\prime}+r_{1,2}^{\prime}\right)} \mathcal{M}_{\mathcal{R}} f_{j}\left(x_{1}, x_{2}\right)+\frac{\left|r_{1,1}^{\prime}-r_{1,1}\right|^{1 / p_{j}^{\prime}}+\left.\left|r_{1,2}^{\prime}-r_{1,2}\right|\right|^{1 / p_{j}}}{\left(r_{2,1}+r_{2,2}\right)^{1 / p_{j}}\left(r_{1,1}^{\prime}+r_{1,2}^{\prime}\right)}\left\|f_{j}\right\|_{L^{p_{j}}\left(\mathbb{R}^{2}\right)} .
\end{aligned}
$$

Then (7.6) follows from this.

Applying Claim 8, we can obtain the following claim immediately.

Claim 9. Let $1<p_{j}<\infty$ and $f_{j} \in L^{p_{j}}\left(\mathbb{R}^{2}\right)$ for $1 \leq j \leq m$. Then for $\left(r_{1,1}, r_{1,2}, r_{2,1}, r_{2,2}\right) \in \overline{\mathbb{R}}_{+}^{4}$ with $r_{1,1}+r_{1,2}>0$ and $r_{2,1}+r_{2,2}>0$,

$$
\lim _{\substack{\left(r_{1,1}^{\prime}, r_{1,2}^{\prime}\right) \in \overline{\mathbb{R}}_{+}^{2} \\\left(r_{1,1}^{\prime}, r_{1,2}^{\prime}\right) \rightarrow\left(r_{1,1}, r_{1,2}\right)}} u_{x, \vec{f}}\left(r_{1,1}^{\prime}, r_{1,2}^{\prime}, r_{2,1}, r_{2,2}\right)=u_{x, \vec{f}}\left(r_{1,1}, r_{1,2}, r_{2,1}, r_{2,2}\right) .
$$

Acknowledgements. This work was completed during the second author was visiting the University of Kansas. The second author is very grateful to both Professor R. H. Torres and the math department for hospitality and stimulation conditions.

\section{REFERENCES}

[1] A. Alfonseca, F. Soria, and A. Vargas, A remark on maximal operators along directions in $\mathbb{R}^{2}$, Math. Res. Lett., 10 (2003), no. 1, 41-49.

[2] J. M. Aldaz and J. Pérez Lázaro, Functions of bounded variation, the derivative of the one dimensional maximal function, and applications to inequalities, Trans. Amer. Math. Soc., 359 (5) (2007), 2443-2461.

[3] J. M. Aldaz and J. Pérez Lázaro, Boundedness and unboundedness results for some maximal operators on functions of bounded variation, J. Math. Anal. Appl., 337 (2008), 130-143.

[4] L. Ambrosio, N. Fusco and D. Pallara, Functions of Bounded Variation and Free Discontinuity Problems, Oxford Math. Monogr., Clarendon Press, Oxford University Press, New York, 2000.

[5] A. Benedek, A. P. Calderón, R. Panzone, Convolution operators on Banach space valued functions, Proc. Nat. Acad. Sci., 48 (1962), 356-365.

[6] J. Bober, E. Carneiro, K. Hughes and L.B. Pierce, On a discrete version of Tanaka's theorem for maximal functions, Proc. Amer. Math. Soc., 140 (5) (2012), 1669-1680.

[7] B. Bojarski and P. Hajłasz, Pointwise inequalities for Sobolev functions and some applications, Studia Math., 106 (1993), 77-92. 
[8] H. Brezis and E. Lieb, A relation between pointwise convergence of functions and convergence of functionals, Proc. Amer. Math. Soc., 88 (1983), 486-490.

[9] E. Carneiro and K. Hughes, On the endpoint regularity of discrete maximal operators, Math. Res. Lett., 19 (6) (2012), 1245-1262.

[10] E. Carneiro and J. Mardid, Derivative bounds for fractional maximal functions, Trans. Amer. Math. Soc., 369 (2017), 4063-4092.

[11] Carneiro, E, Mardid, J, Pierce, L. B: Endpoint Sobolev and BV continuity for maximal operators. J. Funct. Anal., 273 (10) (2017), 3262-3294

[12] E. Carneiro and D. Moreira, On the regularity of maximal operators, Proc. Amer. Math. Soc., 136 (12) (2008), 4395-4404.

[13] E. Carneiro and B. F. Svaiter, On the variation of maximal operators of convolution type, J. Funct. Anal., 265 (2013), 837-865.

[14] A. Córdoba and R. Fefferman, A geometric proof of the strong maximal theorem, Ann. Math., (2) 102 (1) (1975), 91-95.

[15] H. Federer and W. Ziemer, The Lebesgue set of a function whose distribution derivatives are p-th power summable, Indiana Univ. Math. J., 22 (1972), 139-158.

[16] M. Frazier, B. Jawerth and G. Weiss, Littlewood-Paley theory and the study of function spaces, CBMS Reg Conf Ser 9, Amer Math Soc, Providence, RI, 1991.

[17] J. García-Cuerva and J.L. Rubio de Francia, Weighted Norm Inequalities and Related Topic, NorthHolland Mathematics Studies, 116, Mathematical Notes, 104, North Holland Publishing Co., Amsterdam, 1985.

[18] D. Gilbarg and N. S. Trudinger, Elliptic partial differential equations of second order, 2nd edn., SpringerVerlag, Berlin, 1983.

[19] E. Giusti, Minimal Surfaces and Functions of Bounded Variation, Monogr. Math., Birkhäuser, 1984.

[20] L. Grafakos. Classical and Modern Fourier Analysis, Pearson Education, Upper Saddle River, New Jersey, 2004.

[21] L. Grafakos, L. Liu, C. Pérez and R. H. Torres, The multilinear strong maximal function, J. Geom. Anal., 21 (2011), 118-149.

[22] P. Hajłasz, and J. Malý, On approximate differentiability of the maximal function, Proc. Amer. Math. Soc., 138(1) (2010), 165-174.

[23] P. Hajłasz and J. Onninen, On boundedness of maximal functions in Sobolev spaces, Ann. Acad. Sci. Fenn. Math., 29 (1) (2004), 167-176.

[24] G. H. Hardy and J. E. Littlewood, A maximal theorem with function-theoretic applications, Acta Math., 54 (1930), 81-116.

[25] B. Jessen, J. Marcinkiewicz and A. Zygmund, Note on the differentiability of multiple integrals, Fund. Math., 25 (1935), 217-234.

[26] N. H. Katz, A counterexample for maximal oper- ators over a Cantor set of directions, Math. Res. Lett., 3 (1996), no. 4, 527-536.

[27] N. H. Katz, Maximal operators over arbitrary sets of directions, Duke Math. J., 97 (1999), 67-79.

[28] T. Kilpeläinen, J. Kinnunen, O. Martio, Sobolev spaces with zero boundary values on metric spaces, Potential Anal., 12 (2000), 233-247.

[29] J. Kinnunen, The Hardy-Littlewood maximal function of a Sobolev function, Israel J. Math., 100 (1997), 117-124.

[30] J. Kinnunen and P. Lindqvist, The derivative of the maximal function, J. Reine. Angew. Math., 503 (1998), 161-167.

[31] J. Kinnunen and E. Saksman, Regularity of the fractional maximal function, Bull. London Math. Soc.,35 (4) (2003), 529-535.

[32] S. Korry, Boundedness of Hardy-Littlewood maximal operator in the framework of Lizorkin-Triebel spaces, Rev. Mat. Complut., 15 (2) (2002), 401-416.

[33] S. Korry, A class of bounded operators on Sobolev spaces, Arch. Math., 82 (1) 2004, 40-50.

[34] O. Kurka, On the variation of the Hardy-Littlewood maximal function, Ann. Acad. Sci. Fenn. Math., 40 (2015), 109-133.

[35] A. K. Lerner, S. Ombrosi, C. Pérez, R. H. Torres and R. Trujillo-González, New maximal functions and multiple weighted for the multilinear Calderón-Zygmund theory, Adv. Math., 220 (2009), 1222-1264.

[36] J. L. Lewis, On a weak solutions of certain elliptic systems, Commun. Partial Diff. Equa., 18 (1993), 15151537.

[37] F. Liu, A remark on the regularity of the discrete maximal operator, Bull. Austral. Math. Soc., 95 (2017), 108-120. 
[38] F. Liu, Continuity and approximate differentiability of multisublinear fractional maximal functions, Math. Inequal. Appl., 21 (1) (2018), 25-40.

[39] F. Liu, T. Chen and $\mathrm{H}$. Wu, A note on the endpoint regularity of the Hardy-Littlewood maximal functions, Bull. Austral. Math. Soc., 94 (2016), 121-130.

[40] F. Liu and S. Mao, On the regularity of the one-sided Hardy-Littlewood maximal functions, Czech. Math. J., 67 (1) (2017), 219-234.

[41] F. Liu and H. Wu, On the regularity of the multisublinear maximal functions, Canad. Math. Bull., 58 (4) (2015), 808-817.

[42] F. Liu and H. Wu, Endpoint regularity of multisublinear fractional maximal functions, Canad. Math. Bull., 60 (3) (2017), 586-603

[43] F. Liu and $\mathrm{H}$. Wu, Regularity of discrete multisublinear fractional maximal functions, Sci. China Math., 60 (8) (2017), 1461-1476.

[44] F. Liu and H. Wu, On the regularity of maximal operators supported by submanifolds, J. Math. Anal. Appl., 453 (2017), 144-158.

[45] F. Liu and $\mathrm{H}$. Wu, A note on the endpoint regularity of the discrete maximal operator, Proc. Amer. Math. Soc. (to appear). DOI:10.1090/proc/13962.

[46] H. Luiro, Continuity of the maixmal operator in Sobolev spaces, Proc. Amer. Math. Soc., 135 (1) (2007), 243-251.

[47] H. Luiro, On the regularity of the Hardy-Littlewood maximal operator on subdomains of $\mathbb{R}^{n}$, Proc. Edinburgh Math. Soc., 53 (1) (2010), 211-237.

[48] H. Luiro, The variation of the maximal function of a radial function. Ark. Math., (to appear)

[49] J. Madrid, Sharp inequalities for the variation of the discrete maximal function, Bull. Austral. Math. Soc., 95 (2017), 94-107.

[50] A. Nagel, E. M. Stein, and S. Wainger, Differentiation in lacunary directions, Proc. Nat. Acad. Sci USA., 75 (1978), 1060-1062.

[51] H. Tanaka, A remark on the derivative of the one-dimensional Hardy-Littlewood maximal function, Bull. Austral. Math. Soc., 65 (2) (2002), 253-258.

[52] F. Temur, On regularity of the discrete Hardy-Littlewood maximal function, preprint (2015), arxiv.org/abs /1303.3993.

[53] H. Triebel, Theory of Function Spaces, Monogr Math vol 78, Birkhäser Verlag, Basel, 1983.

[54] H. Whitney, On totally differentiable and smooth functions, Pacific. J. Math., 1 (1951), 143-159.

[55] N. Wiener, The ergodic theorem, Duke. Math. J., 5 (1939), 1-18.

[56] K. Yabuta, Triebel-Lizorkin space boundedness of Marcinkiewicz integrals associated to surfaces, Appl. Math. J. Chinese Univ. Ser. B., 30(4), (2015), 418-446.

[57] A. Zygmund, On the differentiability of multiple integrals, Fund. Math., 23 (1934), 143-149.

[58] A. Zygmund, Trigonometric Series, Cambridge, 1959.

Feng Liu, College of Mathematics and Systems Science, Shandong University of Science and Technology, Qingdao, Shandong 266590, People's Republic of China

E-mail address: Fliu@sdust.edu.cn

Qingying Xue, School of Mathematical Sciences, Beijing Normal University, Laboratory of Mathematics and Complex Systems, Ministry of Education, Beijing 100875, People's Republic of China, current address: Department of Mathematics, The University of Kansas, Lawrence, KS 66045, USA

E-mail address: qyxue@bnu.edu.cn

Kôzô Yabuta, Research Center for Mathematical Sciences, Kwansei Gakuin University, Gakuen 2-1, SANDA 669-1337, JAPAN

E-mail address: kyabuta3@kwansei.ac.jp 